\title{
ADAPTIVE TESTING METHOD FOR ERGODIC DIFFUSION PROCESSES BASED ON HIGH FREQUENCY DATA
}

\author{
TETSUYA KAWAI ${ }^{1}$ AND MASAYUKI UCHIDA ${ }^{1,2}$
}

\begin{abstract}
We consider parametric tests for multidimensional ergodic diffusions based on high frequency data. We propose two-step testing method for diffusion parameters and drift parameters. To construct test statistics of the tests, we utilize the adaptive estimator and provide three types of test statistics: likelihood ratio type test, Wald type test and Rao's score type test. It is proved that these test statistics converge in distribution to the chi-squared distribution under null hypothesis and have consistency of the tests against alternatives. Moreover, these test statistics converge in distribution to the non-central chi-squared distribution under local alternatives. We also give some simulation studies of the behavior of the three types of test statistics.
\end{abstract}

\section{INTRODUCTION}

We consider a $d$-dimensional diffusion process satisfying the following stochastic differential equation:

$$
\left\{\begin{array}{l}
d X_{t}=b\left(X_{t}, \beta\right) d t+a\left(X_{t}, \alpha\right) d W_{t} \quad t \in[0, T] \\
X_{0}=x_{0}
\end{array}\right.
$$

where $W_{t}$ is the $r$-dimensional standard Wiener process, $\alpha \in \Theta_{\alpha} \subset \mathbb{R}^{p_{1}}, \beta \in \Theta_{\beta} \subset \mathbb{R}^{p_{2}}, \theta=(\alpha, \beta)$, $\Theta:=\Theta_{\alpha} \times \Theta_{\beta}$ being compact and convex parameter space, $a: \mathbb{R}^{d} \times \Theta_{\alpha} \rightarrow \mathbb{R}^{d} \otimes \mathbb{R}^{r}$ and $b: \mathbb{R}^{d} \times \Theta_{\beta} \rightarrow \mathbb{R}^{d}$ are known except for the parameter $\theta$. We assume the true parameter $\theta_{0}=\left(\alpha_{0}, \beta_{0}\right)$ belongs to $\operatorname{Int}(\Theta)$. The data are discrete observations $\left(X_{t_{i}^{n}}\right)_{0 \leq i \leq n}$, where $t_{i}^{n}=i h_{n}$ for $i=0,1, \ldots, n$, and the discretization step $h_{n}$ satisfies $h_{n} \rightarrow 0, n h_{n} \rightarrow \infty$ and $n h_{n}^{2} \rightarrow 0$ as $n \rightarrow \infty$.

Diffusion processes are used as the mathematical models to describe the random development of the phenomena depending on time in many fields such as physics, neuroscience, meteorology, epidemiology and finance. For these models, the data are discretely observed. The statistical inference for ergodic diffusion process based on discrete observations has been studied by many researchers; see Florens-Zmirou [4, Yoshida [15, 16], Genon-Catalot and Jacod [5], Kessler [8, 9], Uchida and Yoshida 13] and reference therein. In particular, Kessler [8, 9] proposed the adaptive maximum likelihood (ML) type estimator and joint ML type estimator which has asymptotic efficiency under $n h_{n}^{p} \rightarrow 0$, where $p$ is an arbitrary integer with $p \geq 2$. Uchida and Yoshida [13] presented the polynomial type large deviation of adaptive statistical random fields under $n h_{n}^{p} \rightarrow 0$ and moment convergence of the adaptive ML type estimator with asymptotic efficiency.

The parametric testing problem for ergodic diffusions has been studied as the following joint test:

$$
\left\{\begin{array}{l}
H_{0}: \alpha_{1}=\cdots=\alpha_{r_{1}}=0, \quad \beta_{1}=\cdots=\beta_{r_{2}}=0, \quad\left(1 \leq r_{1} \leq p_{1}, 1 \leq r_{2} \leq p_{2}\right) \\
H_{1}: \operatorname{not} H_{0} .
\end{array}\right.
$$

Kitagawa and Uchida [10] proposed three kinds of test statistics (likelihood ratio type test, Wald type test and Rao's score type test) and proved their asymptotic properties. De Gregorio and Iacus [1, 2] constructed the test statistics by means of $\phi$-divergence measure and the empirical $L^{2}$-distance when $r_{1}=p_{1}$ and $r_{2}=p_{2}$.

\footnotetext{
${ }^{1}$ Graduate School of Engineering Science, Osaka University

${ }^{2}$ Center for Mathematical Modeling and Data Science (MMdS), Osaka University and JST CREST

Key words and phrases. Asymptotic theory; Consistency of test; Likelihood ratio test; Rao's score test; Stochastic differential equation; Wald test.
} 
In this paper, we consider the following set of tests instead of (1):

$$
\left\{\begin{array} { l } 
{ H _ { 0 } ^ { ( 1 ) } : \alpha _ { 1 } = \cdots = \alpha _ { r _ { 1 } } = 0 , } \\
{ H _ { 1 } ^ { ( 1 ) } : \operatorname { n o t } H _ { 0 } ^ { ( 1 ) } , }
\end{array} \quad \left\{\begin{array}{l}
H_{0}^{(2)}: \beta_{1}=\cdots=\beta_{r_{2}}=0, \\
H_{1}^{(2)}: \operatorname{not} H_{0}^{(2)} .
\end{array}\right.\right.
$$

The set of tests (2) give more information about parameters than the test (1). The test (1) provides only two interpretations: $H_{0}$ is rejected or $H_{0}$ is not rejected. On the other hand, the tests $(2)$ gives the following four conclusions: (i) $H_{0}^{(1)}$ is rejected and $H_{0}^{(2)}$ is rejected; (ii) $H_{0}^{(1)}$ is rejected and $H_{0}^{(2)}$ is not rejected; (iii) $H_{0}^{(1)}$ is not rejected and $H_{0}^{(2)}$ is rejected; (iv) $H_{0}^{(1)}$ is not rejected and $H_{0}^{(2)}$ is not rejected. We utilize the adaptive ML type estimator of Uchida and Yoshida [13] and construct three types of test statistics. Furthermore, we prove that these test statistics converge in distribution to the chi-squared distribution under null hypothesis, have consistency of the tests under alternatives and converge in distribution to the non-central chi-squared distribution under local alternatives. For the asymptotic null distribution of adaptive test statistics based on local means for noisy ergodic diffusion processes and consistency of the tests under alternatives, see Nakakita and Uchida 12 .

The paper is organized as follows. In Section 2 , notation and assumptions are introduced. In Section 3 , we state main results. Two quasi log likelihood functions are constructed and three kinds of adaptive test statistics are proposed. Moreover, their asymptotic properties are shown. In Section 4 , we give some examples and simulation results of the asymptotic performance for three types of test statistics for 1-dimensional ergodic diffusion processes. Section 5 is devoted to the proofs of the results presented in Section 3.

\section{Notation AND ASSUMPtions}

Let $\partial_{i}:=\partial / \partial x_{i}, \partial_{\alpha_{i}}:=\partial / \partial \alpha_{i}, \partial_{\beta_{i}}:=\partial / \partial \beta_{i}, \partial_{\alpha}:=\left(\partial_{\alpha_{1}}, \ldots, \partial_{\alpha_{p_{1}}}\right)^{\top}, \partial_{\beta}:=\left(\partial_{\beta_{1}}, \ldots, \partial_{\beta_{p_{2}}}\right)^{\top}, \partial_{\alpha}^{2}:=$ $\partial_{\alpha} \partial_{\alpha}^{\top}, \partial_{\beta}^{2}:=\partial_{\beta} \partial_{\beta}^{\top}, \partial_{\alpha \beta}^{2}:=\partial_{\alpha} \partial_{\beta}^{\top}$, where $\top$ is the transpose of a matrix. For $(m \times n)$-matrices $A$ and $B$, it is defined that $A^{\otimes 2}:=A A^{\top},\|A\|^{2}:=\operatorname{tr}\left(A^{\otimes 2}\right), B[A]:=\operatorname{tr}\left(B A^{\top}\right)$, and we set $S(x, \alpha):=(a(x, \alpha))^{\otimes 2}$. For $l \geq 1$ and $m \geq 1$, let $C_{\uparrow}^{l, m}\left(\mathbb{R}^{d} \times \Theta\right)$ be the set of functions $f$ satisfying the following conditions: (i) $f(x, \theta)$ is $l$ times continuously differentiable with respect to $x$; (ii) $f(x, \theta)$ and all its $x$-derivatives up to order $l$ are $m$ times continuously differentiable with respect to $\theta$; (iii) $f(x, \theta)$ and all its derivatives are of polynomial growth in $x$, uniformly in $\theta$. Next, for any positive sequence $u_{n}, R: \Theta \times \mathbb{R} \times \mathbb{R}^{d} \rightarrow \mathbb{R}$ denotes a function with a constant $C>0$ such that for all $\theta \in \Theta$ and $x \in \mathbb{R}^{d},\left|R\left(\theta, u_{n}, x\right)\right| \leq u_{n} C(1+|x|)^{C} . \stackrel{P}{\rightarrow}$ and $\stackrel{d}{\rightarrow}$ indicate convergence in probability and convergence in distribution, respectively. Let $I\left(\theta ; \theta_{0}\right)$ be the $\left(p_{1}+p_{2}\right) \times\left(p_{1}+p_{2}\right)$-matrix defined as

$$
I\left(\theta ; \theta_{0}\right):=\left(\begin{array}{cc}
I_{a}\left(\alpha ; \alpha_{0}\right) & 0 \\
0 & I_{b}\left(\theta ; \theta_{0}\right)
\end{array}\right)
$$

where $I_{a}\left(\alpha ; \alpha_{0}\right)=\left(I_{a}^{(i j)}\left(\alpha ; \alpha_{0}\right)\right)_{1 \leq i, j \leq p_{1}}, I_{b}\left(\theta ; \theta_{0}\right)=\left(I_{b}^{(i j)}\left(\theta ; \theta_{0}\right)\right)_{1 \leq i, j \leq p_{2}}$,

$$
\begin{aligned}
I_{a}^{(i j)}\left(\alpha ; \alpha_{0}\right)= & \frac{1}{2} \int \operatorname{tr}\left(S^{-1}\left(\partial_{\alpha_{i}} S\right) S^{-1}\left(\partial_{\alpha_{j}} S\right) S^{-1}+S^{-1}\left(\partial_{\alpha_{j}} S\right) S^{-1}\left(\partial_{\alpha_{i}} S\right) S^{-1}\right. \\
& \left.-S^{-1}\left(\partial_{\alpha_{i}} \partial_{\alpha_{j}} S\right) S^{-1}\right)(x, \alpha) S\left(x, \alpha_{0}\right) \mu_{\theta_{0}}(d x) \\
& +\frac{1}{2} \int \operatorname{tr}\left(S^{-1}\left(\partial_{\alpha_{i}} \partial_{\alpha_{j}} S\right)-S^{-1}\left(\partial_{\alpha_{i}} S\right) S^{-1}\left(\partial_{\alpha_{j}} S\right)\right)(x, \alpha) \mu_{\theta_{0}}(d x), \\
I_{b}^{(i j)}\left(\theta ; \theta_{0}\right)= & \int\left(b(x, \beta)-b\left(x, \beta_{0}\right)\right)^{\top} S^{-1}(x, \alpha)\left(\partial_{\beta_{i}} \partial_{\beta_{j}} b(x, \beta)\right) \mu_{\theta_{0}}(d x) \\
& +\int\left(\partial_{\beta_{i}} b(x, \beta)\right)^{\top} S^{-1}(x, \alpha)\left(\partial_{\beta_{j}} b(x, \beta)\right) \mu_{\theta_{0}}(d x) .
\end{aligned}
$$

We make the following assumptions.

A1 There exists $C>0$ such that for all $x, y \in \mathbb{R}^{d}$,

$$
\sup _{\alpha \in \Theta_{\alpha}}\|a(x, \alpha)-a(y, \alpha)\|+\sup _{\beta \in \Theta_{\beta}}|b(x, \beta)-b(y, \beta)| \leq C|x-y| .
$$

A2 The diffusion process $X$ is ergodic with its invariant measure $\mu_{\theta_{0}}(d x)$. 
A3 $\inf _{x, \alpha} \operatorname{det}(S(x, \alpha))>0$.

A4 For all $p \geq 0, \sup E_{\theta}\left[\left|X_{t}\right|^{p}\right]<\infty$.

A5 $a \in C_{\uparrow}^{4,3}\left(\mathbb{R}^{d} \times \Theta_{\alpha}^{t}\right), b \in C_{\uparrow}^{4,3}\left(\mathbb{R}^{d} \times \Theta_{\beta}\right)$.

A6 $b(x, \beta)=b\left(x, \beta_{0}\right)$ for $\mu_{\theta_{0}}$ a.s. all $x \Rightarrow \beta=\beta_{0}$.

$S(x, \alpha)=S\left(x, \alpha_{0}\right)$ for $\mu_{\theta_{0}}$ a.s. all $x \Rightarrow \alpha=\alpha_{0}$.

A7 $I\left(\theta_{0} ; \theta_{0}\right)$ is non-singular.

\section{MAIN RESULTS}

\subsection{Asymptotic distribution under null hypothesis.}

For $1 \leq r_{1} \leq p_{1}$ and $1 \leq r_{2} \leq p_{2}$, we consider the following set of tests:

$$
\left\{\begin{array} { l } 
{ H _ { 0 } ^ { ( 1 ) } : \alpha _ { 1 } = \cdots = \alpha _ { r _ { 1 } } = 0 , } \\
{ H _ { 1 } ^ { ( 1 ) } : \operatorname { n o t } H _ { 0 } ^ { ( 1 ) } , }
\end{array} \quad \left\{\begin{array}{l}
H_{0}^{(2)}: \beta_{1}=\cdots=\beta_{r_{2}}=0 \\
H_{1}^{(2)}: \operatorname{not} H_{0}^{(2)} .
\end{array}\right.\right.
$$

Let $\tilde{\Theta}_{\alpha}:=\left\{\alpha \in \Theta_{\alpha} \mid \alpha\right.$ holds $\left.H_{0}^{(1)}\right\}, \tilde{\Theta}_{\beta}:=\left\{\beta \in \Theta_{\beta} \mid \beta\right.$ holds $\left.H_{0}^{(2)}\right\}, \tilde{\Theta}:=\tilde{\Theta}_{\alpha} \times \tilde{\Theta}_{\beta}$.

The quasi log likelihood functions are defined as follows:

$$
\begin{aligned}
U_{n}^{(1)}(\alpha) & :=-\frac{1}{2} \sum_{i=1}^{n}\left\{h_{n}^{-1} S^{-1}\left(X_{t_{i-1}^{n}}, \alpha\right)\left[\left(X_{t_{i}^{n}}-X_{t_{i-1}^{n}}\right)^{\otimes 2}\right]+\log \operatorname{det} S\left(X_{t_{i-1}^{n}}, \alpha\right)\right\}, \\
U_{n}^{(2)}(\beta \mid \bar{\alpha}): & =-\frac{1}{2} \sum_{i=1}^{n}\left\{h_{n}^{-1} S^{-1}\left(X_{t_{i-1}^{n}}, \bar{\alpha}\right)\left[\left(X_{t_{i}^{n}}-X_{t_{i-1}^{n}}-h_{n} b\left(X_{t_{i-1}^{n}}, \beta\right)\right)^{\otimes 2}\right]\right\} .
\end{aligned}
$$

The adaptive ML type estimator under $\Theta, \hat{\theta}_{n}=\left(\hat{\alpha}_{n}, \hat{\beta}_{n}\right)$ and the adaptive ML type estimator under $\tilde{\Theta}$, $\tilde{\theta}_{n}=\left(\tilde{\alpha}_{n}, \tilde{\beta}_{n}\right)$ are defined as

$$
\begin{gathered}
\hat{\alpha}_{n}:=\underset{\alpha \in \Theta_{\alpha}}{\arg \sup } U_{n}^{(1)}(\alpha), \quad \tilde{\alpha}_{n}:=\underset{\alpha \in \tilde{\Theta}_{\alpha}}{\arg \sup } U_{n}^{(1)}(\alpha), \\
\hat{\beta}_{n}:=\underset{\beta \in \Theta_{\beta}}{\arg \sup } U_{n}^{(2)}(\beta \mid \hat{\alpha}), \quad \tilde{\beta}_{n}:=\underset{\beta \in \tilde{\Theta}_{\beta}}{\arg \sup } U_{n}^{(2)}(\beta \mid \hat{\alpha}) .
\end{gathered}
$$

Next we set

$$
\begin{gathered}
I_{a, n}(\alpha):=-\frac{1}{n} \partial_{\alpha}^{2} U_{n}^{(1)}(\alpha), \quad I_{b, n}(\beta \mid \alpha):=-\frac{1}{n h_{n}} \partial_{\beta}^{2} U_{n}^{(2)}(\beta \mid \alpha), \\
J_{a, n}(\alpha):=\left\{I_{a, n}(\alpha) \text { is non-singular }\right\}, J_{b, n}(\bar{\alpha}, \beta):=\left\{I_{b, n}(\beta \mid \bar{\alpha}) \text { is non-singular }\right\}, \\
\bar{I}_{a, n}(\alpha):=\left\{\begin{array}{ll}
I_{a, n}^{-1}(\alpha) & \left(\omega \in J_{a, n}(\alpha)\right), \\
E_{p_{1}} & \left(\omega \in J_{a, n}^{C}(\alpha)\right),
\end{array} \bar{I}_{b, n}(\beta \mid \bar{\alpha}):= \begin{cases}I_{b, n}^{-1}(\beta \mid \bar{\alpha}) & \left(\omega \in J_{b, n}(\bar{\alpha}, \beta)\right), \\
E_{p_{2}} & \left(\omega \in J_{b, n}^{C}(\bar{\alpha}, \beta)\right) .\end{cases} \right.
\end{gathered}
$$

We define the likelihood ratio, Wald and Rao type test statistics $\Lambda_{n}^{(i)}, W_{n}^{(i)}, R_{n}^{(i)}(i=1,2)$ as follows:

$$
\begin{aligned}
\Lambda_{n}^{(1)} & :=-2\left(U_{n}^{(1)}\left(\tilde{\alpha}_{n}\right)-U_{n}^{(1)}\left(\hat{\alpha}_{n}\right)\right), \quad \Lambda_{n}^{(2)}:=-2\left(U_{n}^{(2)}\left(\tilde{\beta}_{n} \mid \hat{\alpha}_{n}\right)-U_{n}^{(2)}\left(\hat{\beta}_{n} \mid \hat{\alpha}_{n}\right)\right), \\
W_{n}^{(1)} & :=n\left(\hat{\alpha}_{n}-\tilde{\alpha}_{n}\right)^{\top} I_{a, n}\left(\hat{\alpha}_{n}\right)\left(\hat{\alpha}_{n}-\tilde{\alpha}_{n}\right), \quad W_{n}^{(2)}:=n h_{n}\left(\hat{\beta}_{n}-\tilde{\beta}_{n}\right)^{\top} I_{b, n}\left(\hat{\beta}_{n} \mid \hat{\alpha}_{n}\right)\left(\hat{\beta}_{n}-\tilde{\beta}_{n}\right), \\
R_{n}^{(1)} & :=n^{-1}\left(\partial_{\alpha} U_{n}^{(1)}\left(\tilde{\alpha}_{n}\right)\right)^{\top} \bar{I}_{a, n}\left(\hat{\alpha}_{n}\right) \partial_{\alpha} U_{n}^{(1)}\left(\tilde{\alpha}_{n}\right), \\
R_{n}^{(2)} & :=\left(n h_{n}\right)^{-1}\left(\partial_{\beta} U_{n}^{(2)}\left(\tilde{\beta}_{n} \mid \hat{\alpha}_{n}\right)\right)^{\top} \bar{I}_{b, n}\left(\hat{\beta}_{n} \mid \hat{\alpha}_{n}\right) \partial_{\beta} U_{n}^{(2)}\left(\tilde{\beta}_{n} \mid \hat{\alpha}_{n}\right),
\end{aligned}
$$

where $\Lambda_{n}^{(1)}, W_{n}^{(1)}$ and $R_{n}^{(1)}$ are used in testing $\alpha$ and $\Lambda_{n}^{(2)}, W_{n}^{(2)}$ and $R_{n}^{(2)}$ are used in testing $\beta$.

The following gives asymptotic distributions of these test statistics under null hypothesis.

Theorem 1 Assume A1-A7. If $h_{n} \rightarrow 0, n h_{n} \rightarrow \infty$ and $n h_{n}^{2} \rightarrow 0$, then

(i) $\Lambda_{n}^{(1)} \stackrel{d}{\rightarrow} \chi_{r_{1}}^{2} \quad\left(\right.$ under $\left.H_{0}^{(1)}\right), \quad \Lambda_{n}^{(2)} \stackrel{d}{\rightarrow} \chi_{r_{2}}^{2} \quad$ (under $H_{0}^{(2)}$ ),

(ii) $W_{n}^{(1)} \stackrel{d}{\rightarrow} \chi_{r_{1}}^{2} \quad\left(\right.$ under $\left.H_{0}^{(1)}\right), \quad W_{n}^{(2)} \stackrel{d}{\rightarrow} \chi_{r_{2}}^{2} \quad$ (under $H_{0}^{(2)}$ ),

(iii) $R_{n}^{(1)} \stackrel{d}{\rightarrow} \chi_{r_{1}}^{2} \quad\left(\right.$ under $\left.H_{0}^{(1)}\right), \quad R_{n}^{(2)} \stackrel{d}{\rightarrow} \chi_{r_{2}}^{2} \quad$ (under $H_{0}^{(2)}$ ). 
Remark 1 When we perform the parametric tests for $\theta=(\alpha, \beta)$, the testing procedure is as follows:

(1) test $H_{0}^{(1)}$ v.s. $H_{1}^{(1)}$;

(2) test $H_{0}^{(2)}$ v.s. $H_{1}^{(2)}$ regardless of the result of (1).

This testing method provides four interpretations, which gives more information about the parameters than the joint test by Kitagawa and Uchida [10].

\subsection{Consistency of tests.}

Next we consider under alternatives $H_{1}^{(1)}$ or $H_{1}^{(2)}$. To emphasize that we consider under alternatives, $\theta_{1}^{*}=$ $\left(\alpha_{1}^{*}, \beta_{1}^{*}\right)$ denotes the true parameter under alternatives. We define an optimal parameter $\theta^{*}=\left(\alpha^{*}, \beta^{*}\right) \in \tilde{\Theta}$ as

$$
\alpha^{*}:=\underset{\alpha \in \tilde{\Theta}_{\alpha}}{\arg \sup } \bar{U}_{1}\left(\alpha ; \alpha_{1}^{*}\right), \quad \beta^{*}:=\underset{\alpha \in \tilde{\Theta}_{\beta}}{\arg \sup } \bar{U}_{2}\left(\alpha_{1}^{*}, \beta ; \beta_{1}^{*}\right),
$$

where

$$
\begin{aligned}
\bar{U}_{1}\left(\alpha ; \alpha_{1}^{*}\right) & :=-\frac{1}{2} \int\left(\operatorname{tr}\left(S\left(x, \alpha_{1}^{*}\right) S^{-1}(x, \alpha)\right)+\log \operatorname{det} S(x, \alpha)\right) \mu_{\theta_{1}^{*}}(d x), \\
\bar{U}_{2}\left(\alpha, \beta ; \beta_{1}^{*}\right) & :=-\frac{1}{2} \int S^{-1}(x, \alpha)\left[\left(b\left(x, \beta_{1}^{*}\right)-b(x, \beta)\right)^{\otimes 2}\right] \mu_{\theta_{1}^{*}}(d x) .
\end{aligned}
$$

Remark 2 It always true that $\alpha_{1}^{*} \neq \alpha^{*}$. Since $\alpha_{1}^{*}$ is the true value under $H_{1}^{(1)}$, there exists $i \in\left\{1, \ldots, r_{1}\right\}$ such that $\alpha_{i} \neq 0$. On the other hand, $\alpha^{*}$ is the optimal value under $H_{1}^{(0)}$, hence $\alpha_{i}=0 \quad\left(i=1, \ldots, r_{1}\right)$. Similarly, it always holds $\beta_{1}^{*} \neq \beta^{*}$.

We assume the following conditions.

B1 (a) For all $\varepsilon>0$,

$$
\sup _{\left\{\alpha \in \tilde{\Theta}_{\alpha} ;\left|\alpha-\alpha^{*}\right| \geq \varepsilon\right\}}\left(\bar{U}_{1}\left(\alpha ; \alpha_{1}^{*}\right)-\bar{U}_{1}\left(\alpha^{*} ; \alpha_{1}^{*}\right)\right)<0 .
$$

(b) For all $\varepsilon>0$,

$$
\sup _{\left\{\beta \in \tilde{\Theta}_{\beta} ;\left|\beta-\beta^{*}\right| \geq \varepsilon\right\}}\left(\bar{U}_{2}\left(\alpha_{1}^{*}, \beta ; \beta_{1}^{*}\right)-\bar{U}_{2}\left(\alpha_{1}^{*}, \beta^{*} ; \beta_{1}^{*}\right)\right)<0 .
$$

B2 (a) For all $\alpha \in \Theta_{\alpha}, I_{a}\left(\alpha ; \alpha_{1}^{*}\right)$ is non-singular.

(b) For all $\beta \in \Theta_{\beta}, I_{b}\left(\alpha_{1}^{*}, \beta ; \theta_{1}^{*}\right)$ is non-singular.

The following gives the consistency of tests.

Theorem 2 Assume A1-A7 and B1. If $h_{n} \rightarrow 0$ and $n h_{n} \rightarrow \infty$, then for all $\varepsilon \in(0,1)$,

(i) $P\left(\Lambda_{n}^{(1)} \geq \chi_{r_{1}, \varepsilon}^{2}\right) \rightarrow 1 \quad\left(\right.$ under $\left.H_{1}^{(1)}\right), \quad P\left(\Lambda_{n}^{(2)} \geq \chi_{r_{2}, \varepsilon}^{2}\right) \rightarrow 1 \quad\left(\right.$ under $\left.H_{1}^{(2)}\right)$,

(ii) $P\left(W_{n}^{(1)} \geq \chi_{r_{1}, \varepsilon}^{2}\right) \rightarrow 1 \quad$ (under $\left.H_{1}^{(1)}\right), \quad P\left(W_{n}^{(2)} \geq \chi_{r_{2}, \varepsilon}^{2}\right) \rightarrow 1 \quad$ (under $\left.H_{1}^{(2)}\right)$,

(iii) $P\left(R_{n}^{(1)} \geq \chi_{r_{1}, \varepsilon}^{2}\right) \rightarrow 1 \quad$ (under $H_{1}^{(1)}$ and $\mathbf{B 2}$-(a)),

$P\left(R_{n}^{(2)} \geq \chi_{r_{2}, \varepsilon}^{2}\right) \rightarrow 1 \quad$ (under $H_{1}^{(2)}$ and B2-(b)),

where $\chi_{p, \varepsilon}^{2}$ denotes the upper $\varepsilon$ point of chi-squared distribution with $p$ degrees of freedom.

\subsection{Asymptotic distribution under local alternatives.}

For $u_{\alpha} \in \mathbb{R}^{p_{1}}$ and $u_{\beta} \in \mathbb{R}^{p_{2}}$, we consider the following tests.

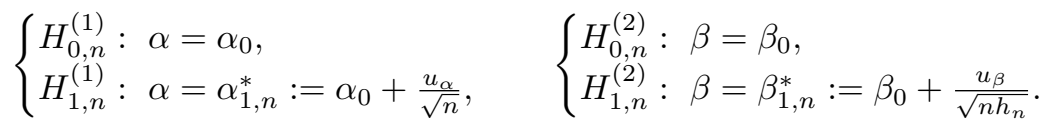

Let $\theta_{1, n}^{*}$ denote the true parameter under local alternatives. We assume the following condition.

C1 $P_{\theta_{1, n}^{*}}$ is contiguous with respect to $P_{\theta_{0}}$. That is, for sequence of sets $A_{n}$,

$$
\lim _{n \rightarrow \infty} P_{\theta_{0}}\left(A_{n}\right)=0 \Rightarrow \lim _{n \rightarrow \infty} P_{\theta_{1, n}^{*}}\left(A_{n}\right)=0
$$


Remark 3 (i) Let $\bar{\alpha}$ denote the true parameter of $\alpha$ under $H_{0, n}^{(2)}$ or $H_{1, n}^{(2)}$, and $\bar{\beta}$ denote the true parameter of $\beta$ under $H_{0, n}^{(1)}$ or $H_{1, n}^{(1)}$. By using these symbols, $\mathbf{C} 1$ is applied as follows:

(I) If we test for $\alpha$, then $\theta_{0}=\left(\alpha_{0}, \bar{\beta}\right), \theta_{1, n}^{*}=\left(\alpha_{1, n}^{*}, \bar{\beta}\right)$.

(II) If we test for $\beta$, then $\theta_{0}=\left(\bar{\alpha}, \beta_{0}\right), \theta_{1, n}^{*}=\left(\bar{\alpha}, \beta_{1, n}^{*}\right)$.

(ii) A sufficient condition of $\mathbf{C 1}$ is local asymptotic normality (LAN), and a sufficient condition of LAN is as follows: A1-A5 and

(I) There exists $C>0$ such that for all $x \in \mathbb{R}^{d}$ and $(\alpha, \beta) \in \Theta$, $|b(x, \beta)| \leq C(1+|x|), \quad\left|\partial_{i} b(x, \beta)\right|+|a(x, \alpha)|+\left|\partial_{i} a(x, \alpha)\right| \leq C$.

(II) There exists $c_{0}>0$ and $K>0$ such that for all $(x, \beta) \in \mathbb{R}^{d} \times \Theta_{\beta}$, $b(x, \beta)^{\top} x \leq-c_{0}|x|^{2}+K$.

For details of the relation between LAN and C1, see, for example, van der Vaart 14. For sufficient conditions of LAN, see Gobet[6].

Theorem 3 Assume A1-A7 and C1. If $h_{n} \rightarrow 0, n h_{n} \rightarrow \infty$ and $n h_{n}^{2} \rightarrow 0$, then

(i) $\Lambda_{n}^{(1)} \stackrel{d}{\rightarrow} \chi_{p_{1}}^{2}\left(u_{\alpha}^{\top} I_{a}\left(\alpha_{0} ; \alpha_{0}\right) u_{\alpha}\right) \quad\left(\right.$ under $\left.H_{1, n}^{(1)}\right), \quad \Lambda_{n}^{(2)} \stackrel{d}{\rightarrow} \chi_{p_{2}}^{2}\left(u_{\beta}^{\top} I_{b}\left(\theta_{0} ; \theta_{0}\right) u_{\beta}\right) \quad$ (under $\left.H_{1, n}^{(2)}\right)$,

(ii) $W_{n}^{(1)} \stackrel{d}{\rightarrow} \chi_{p_{1}}^{2}\left(u_{\alpha}^{\top} I_{a}\left(\alpha_{0} ; \alpha_{0}\right) u_{\alpha}\right) \quad$ (under $\left.H_{1, n}^{(1)}\right), \quad W_{n}^{(2)} \stackrel{d}{\rightarrow} \chi_{p_{2}}^{2}\left(u_{\beta}^{\top} I_{b}\left(\theta_{0} ; \theta_{0}\right) u_{\beta}\right) \quad$ (under $\left.H_{1, n}^{(2)}\right)$,

(iii) $R_{n}^{(1)} \stackrel{d}{\rightarrow} \chi_{p_{1}}^{2}\left(u_{\alpha}^{\top} I_{a}\left(\alpha_{0} ; \alpha_{0}\right) u_{\alpha}\right) \quad\left(\right.$ under $\left.H_{1, n}^{(1)}\right), \quad R_{n}^{(2)} \stackrel{d}{\rightarrow} \chi_{p_{2}}^{2}\left(u_{\beta}^{\top} I_{b}\left(\theta_{0} ; \theta_{0}\right) u_{\beta}\right) \quad$ (under $\left.H_{1, n}^{(2)}\right)$,

where $\chi_{p}^{2}(c)$ denotes the non-central chi-squared distribution with $p$ degrees of freedom and noncentrality parameter $c$.

\section{Examples AND Simulations}

\subsection{Model 1.}

We consider the following 1-dimensional Ornstein-Uhlenbeck process:

$$
\left\{\begin{array}{l}
d X_{t}=-\left(X_{t}-\beta\right) d t+\alpha d W_{t}, \\
X_{0}=1.0
\end{array}\right.
$$

We simulate the asymptotic performance of the three types of test statistics: likelihood ratio type, Wald type and Rao's score type. In model (3), we deal with the following hypothesis tests:

$$
\left\{\begin{array} { l } 
{ H _ { 0 } ^ { ( 1 ) } : \alpha = 1 . 0 , } \\
{ H _ { 1 } ^ { ( 1 ) } : \alpha \neq 1 . 0 , }
\end{array} \quad \left\{\begin{array}{l}
H_{0}^{(2)}: \beta=2.0, \\
H_{1}^{(2)}: \beta \neq 2.0 .
\end{array}\right.\right.
$$

These tests derive the four kinds of results as follows:

Case 1. Neither $\alpha$ nor $\beta$ is rejected;

Case 2. $\alpha$ is not rejected, but $\beta$ is rejected;

Case 3. $\alpha$ is rejected, but $\beta$ is not rejected;

Case 4. Both $\alpha$ and $\beta$ are rejected;

We choice the true parameter $\theta^{*}=\left(\alpha^{*}, \beta^{*}\right)$ from $\{(1.0,2.0),(1.0,2.5),(1.1,2.0),(1.1,2.5)\}$, where $\theta^{*}$ corresponds to the true parameter of Cases 1-4. Let $n$ be fix and $h_{n}=n^{-2 / 3}$, which satisfies the conditions $n h_{n}=n^{1 / 3} \rightarrow \infty$ and $n h_{n}^{2}=n^{-1 / 3} \rightarrow 0$ as $n \rightarrow \infty$. In this simulation, we consider the cases of $n=10^{4}, 10^{5}$ and $10^{6}$. Let the significance level denote $\varepsilon=0.05$ and each test is rejected when the realization of each test statistic is greater than $\chi_{1,0.05}^{2}$. The simulation is repeated 1000 times.

Tables 1. 4 show the number of counts of Cases 1-4 selected by the tests (4), where the true parameter $\theta^{*}$ corresponds to each case. In all of the tables, the true case is most often selected as $n$ increases. In Table 1, the percent of misidentification is about 10 percent, which is the sum of the significance levels of the considering tests. Figures 1,8 are simulation results of the histograms and the empirical distributions of the three types of test statistics in Theorem 1. Theoretically, these test statistics converge in distribution to $\chi_{1}^{2}$, and we see from all of the figures that these test statistics have good behavior. Tables 5 / 8 show the empirical sizes of the three test statistics when the null hypothesis is true. 
TABle 1. Results of test statistics in Case 1: $\left(\alpha^{*}, \beta^{*}\right)=(1.0,2.0)$.

\begin{tabular}{ccc|c|cccc}
$n$ & $h_{n}$ & $n h_{n}$ & Test type & Case 1 & Case 2 & Case 3 & Case 4 \\
\hline \hline \multirow{3}{*}{$10^{4}$} & \multirow{2}{*}{$2.15 \times 10^{-3}$} & \multirow{2}{*}{21.5} & Likelihood & 890 & 53 & 53 & 4 \\
& & Wald & 891 & 54 & 52 & 3 \\
& & Rao & 891 & 52 & 52 & 5 \\
\hline \multirow{3}{*}{$10^{5}$} & \multirow{3}{*}{$4.64 \times 10^{-4}$} & \multirow{2}{*}{46.4} & Likelihood & 897 & 50 & 50 & 3 \\
& & & Wald & 897 & 50 & 50 & 3 \\
& & Rao & 897 & 50 & 50 & 3 \\
\hline \multirow{3}{*}{$10^{6}$} & \multirow{3}{*}{$1.00 \times 10^{-4}$} & \multirow{2}{*}{100} & Likelihood & 900 & 49 & 47 & 4 \\
& & & Wald & 900 & 49 & 47 & 4 \\
& & Rao & 901 & 49 & 46 & 4
\end{tabular}

TABLe 2. Results of test statistics in Case 2: $\left(\alpha^{*}, \beta^{*}\right)=(1.0,2.5)$.

\begin{tabular}{ccc|c|cccc}
$n$ & $h_{n}$ & $n h_{n}$ & Test type & Case 1 & Case 2 & Case 3 & Case 4 \\
\hline \hline \multirow{3}{*}{$10^{4}$} & \multirow{3}{*}{$2.15 \times 10^{-3}$} & \multirow{2}{*}{21.5} & Likelihood & 352 & 590 & 23 & 35 \\
& & Wald & 353 & 591 & 22 & 34 \\
& & Rao & 352 & 590 & 23 & 35 \\
\hline \multirow{3}{*}{$10^{5}$} & \multirow{3}{*}{$4.64 \times 10^{-4}$} & \multirow{2}{*}{46.4} & Likelihood & 74 & 881 & 3 & 42 \\
& & & Wald & 74 & 883 & 3 & 40 \\
& & Rao & 74 & 880 & 3 & 43 \\
\hline \multirow{3}{*}{$10^{6}$} & \multirow{3}{*}{$1.00 \times 10^{-4}$} & \multirow{2}{*}{100} & Likelihood & 1 & 950 & 0 & 49 \\
& & & Wald & 1 & 950 & 0 & 49 \\
& & & Rao & 1 & 950 & 0 & 49
\end{tabular}

TABLE 3. Results of test statistics in Case 3: $\left(\alpha^{*}, \beta^{*}\right)=(1.1,2.0)$.

\begin{tabular}{ccc|c|cccc}
$n$ & $h_{n}$ & $n h_{n}$ & Test type & Case 1 & Case 2 & Case 3 & Case 4 \\
\hline \hline \multirow{3}{*}{$10^{4}$} & \multirow{3}{*}{$2.15 \times 10^{-3}$} & \multirow{2}{*}{21.5} & Likelihood & 0 & 0 & 956 & 44 \\
& & Wald & 0 & 0 & 956 & 44 \\
& & Rao & 0 & 0 & 956 & 44 \\
\hline \multirow{3}{*}{$10^{5}$} & \multirow{3}{*}{$4.64 \times 10^{-4}$} & \multirow{2}{*}{46.4} & Likelihood & 0 & 0 & 954 & 46 \\
& & & Wald & 0 & 0 & 954 & 46 \\
& & Rao & 0 & 0 & 954 & 46 \\
\hline \multirow{3}{*}{$10^{6}$} & \multirow{3}{*}{$1.00 \times 10^{-4}$} & \multirow{2}{*}{100} & Likelihood & 0 & 0 & 955 & 45 \\
& & & Wald & 0 & 0 & 955 & 45 \\
& & Rao & 0 & 0 & 955 & 45
\end{tabular}

TABle 4. Results of test statistics in Case 4: $\left(\alpha^{*}, \beta^{*}\right)=(1.1,2.5)$.

\begin{tabular}{ccc|c|cccc}
$n$ & $h_{n}$ & $n h_{n}$ & Test type & Case 1 & Case 2 & Case 3 & Case 4 \\
\hline \hline \multirow{3}{*}{$10^{4}$} & \multirow{3}{*}{$2.15 \times 10^{-3}$} & \multirow{2}{*}{21.5} & Likelihood & 0 & 0 & 425 & 575 \\
& & Wald & 0 & 0 & 425 & 575 \\
& & Rao & 0 & 0 & 425 & 575 \\
\hline \multirow{3}{*}{$10^{5}$} & \multirow{3}{*}{$4.64 \times 10^{-4}$} & \multirow{2}{*}{46.4} & Likelihood & 0 & 0 & 114 & 886 \\
& & & Wald & 0 & 0 & 114 & 886 \\
& & Rao & 0 & 0 & 114 & 886 \\
\hline \multirow{3}{*}{$10^{6}$} & \multirow{3}{*}{$1.00 \times 10^{-4}$} & \multirow{2}{*}{100} & Likelihood & 0 & 0 & 2 & 998 \\
& & & Wald & 0 & 0 & 2 & 998 \\
& & & Rao & 0 & 0 & 2 & 998
\end{tabular}



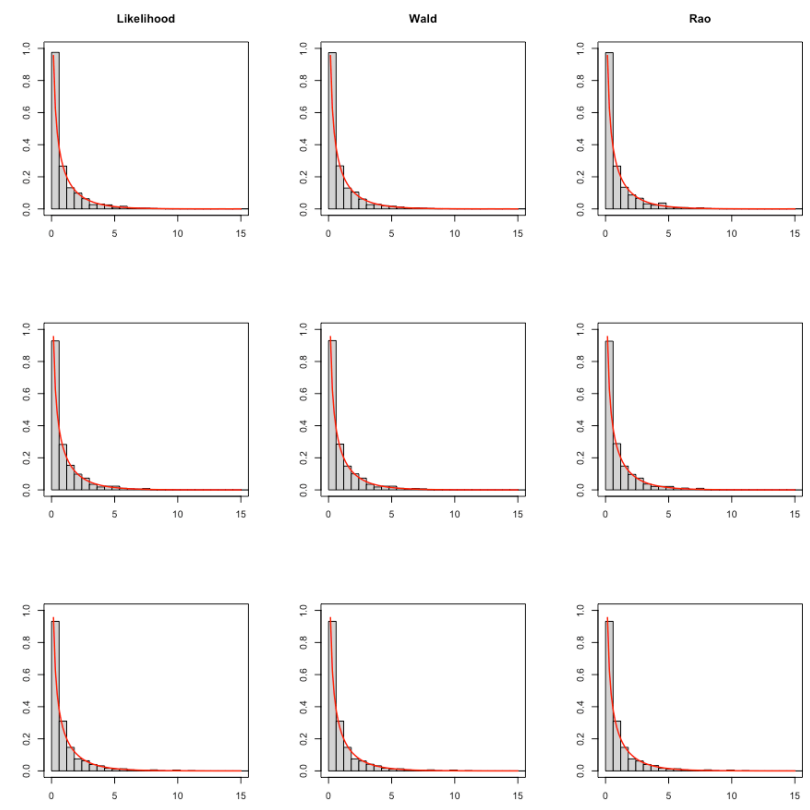

Figure 1. Histograms of the three types of test statistics for $\alpha$ in Case 1. Each row of figures corresponds to the case of $n=10^{4}$ (upper), $10^{5}$ (middle) and $10^{6}$ (bottom) and each column of figures corresponds to Likelihood ratio (left), Wald (middle) and Rao (right) type test statistics. The red line is the probability density function of $\chi_{1}^{2}$.
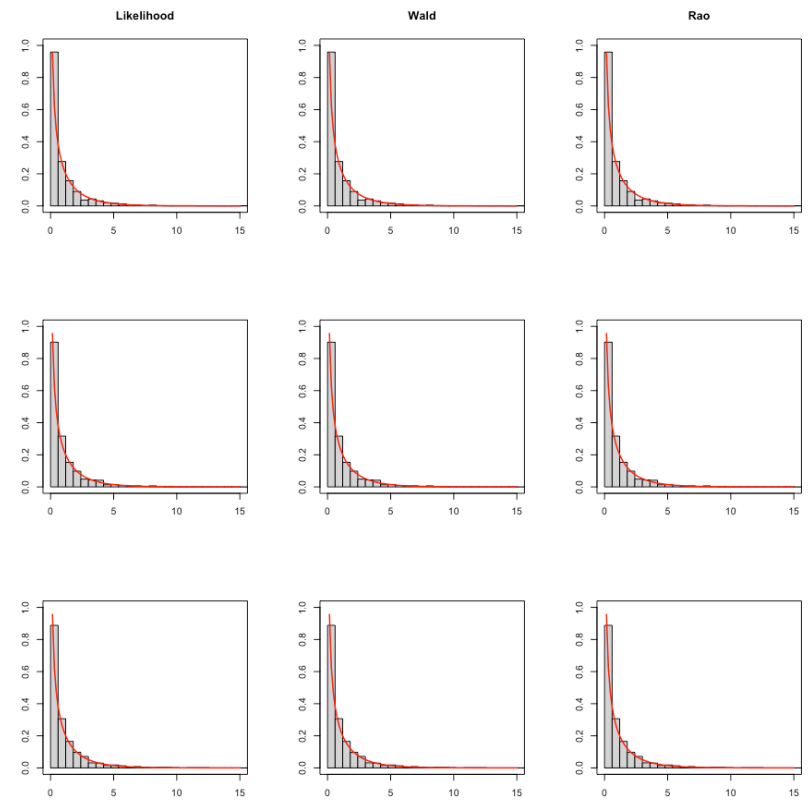

Figure 2. Histograms of the three types of test statistics for $\beta$ in Case 1. Each row of figures corresponds to the case of $n=10^{4}$ (upper), $10^{5}$ (middle) and $10^{6}$ (bottom) and each column of figures corresponds to Likelihood ratio (left), Wald (middle) and Rao (right) type test statistics. The red line is the probability density function of $\chi_{1}^{2}$. 

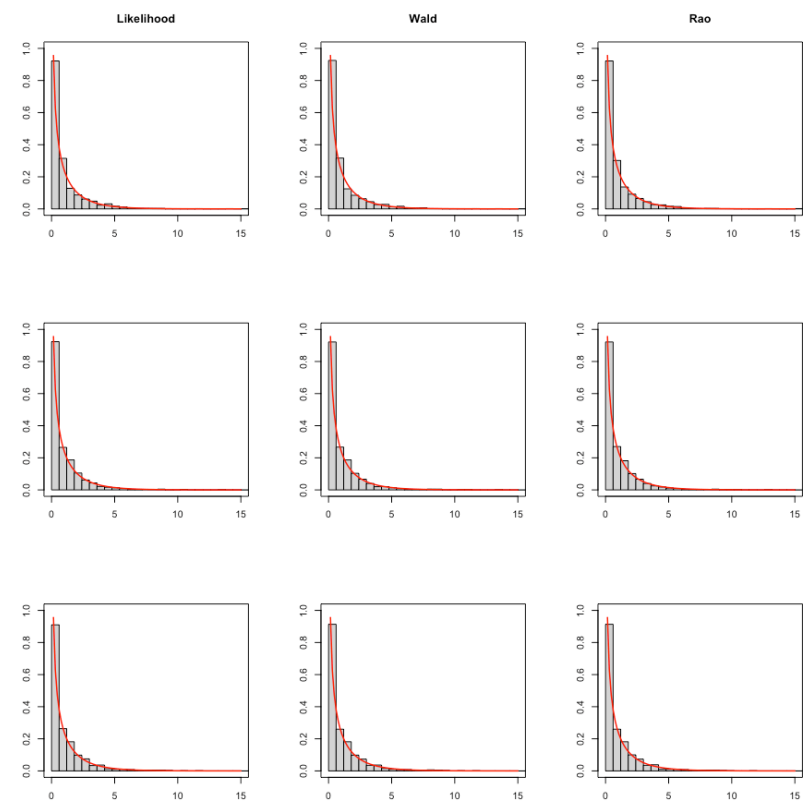

Figure 3. Histograms of the three types of test statistics for $\alpha$ in Case 2. Each row of figures corresponds to the case of $n=10^{4}$ (upper), $10^{5}$ (middle) and $10^{6}$ (bottom) and each column of figures corresponds to Likelihood ratio (left), Wald (middle) and Rao (right) type test statistics. The red line is the probability density function of $\chi_{1}^{2}$.
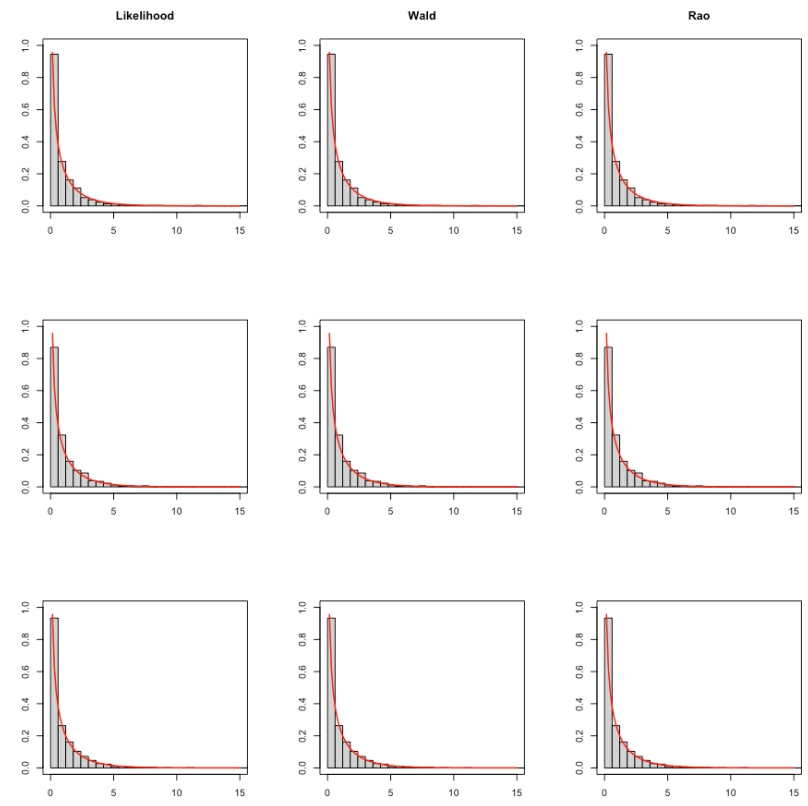

Figure 4. Histograms of the three types of test statistics for $\beta$ in Case 3. Each row of figures corresponds to the case of $n=10^{4}$ (upper), $10^{5}$ (middle) and $10^{6}$ (bottom) and each column of figures corresponds to Likelihood ratio (left), Wald (middle) and Rao (right) type test statistics. The red line is the probability density function of $\chi_{1}^{2}$. 

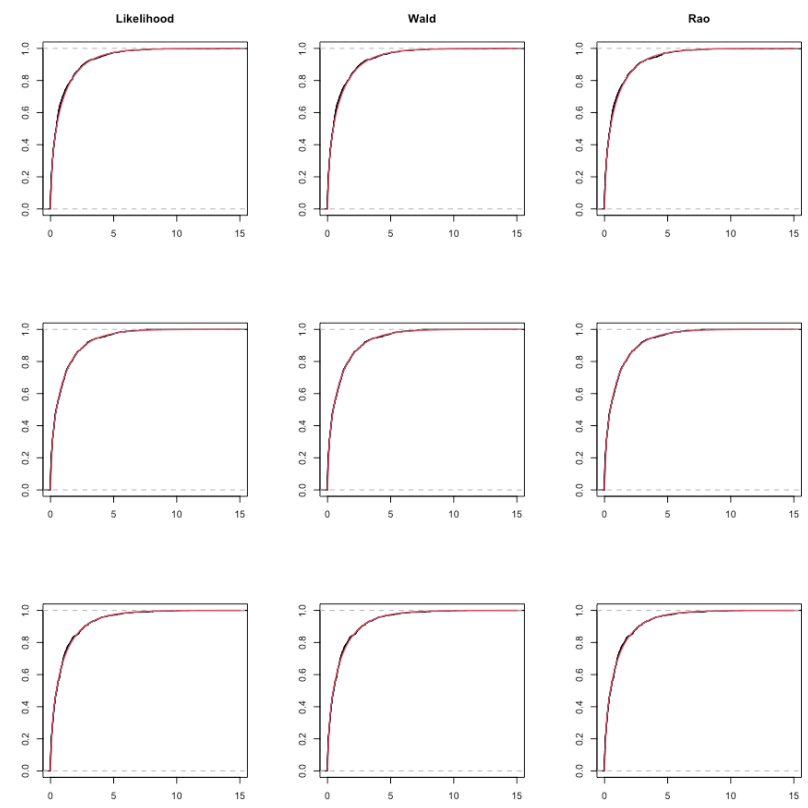

FiguRE 5. Empirical distributions of the three types of test statistics for $\alpha$ in Case 1. Each row of figures corresponds to the case of $n=10^{4}$ (upper), $10^{5}$ (middle) and $10^{6}$ (bottom) and each column of figures corresponds to Likelihood ratio (left), Wald (middle) and Rao (right) type test statistics. The red line is the cumulative distribution function of $\chi_{1}^{2}$.
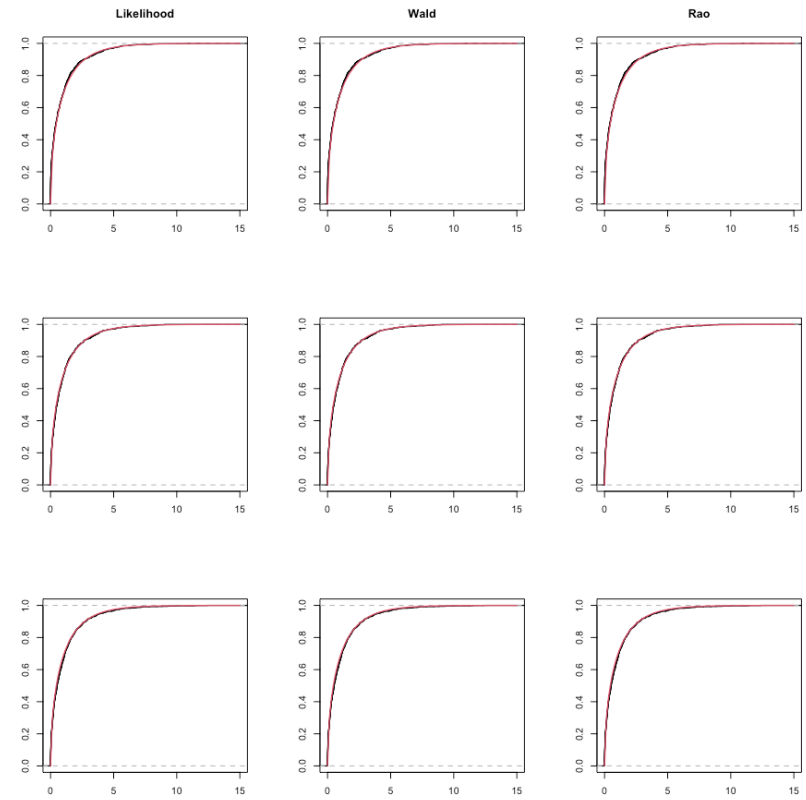

Figure 6. Empirical distributions of the three types of test statistics for $\beta$ in Case 1. Each row of figures corresponds to the case of $n=10^{4}$ (upper), $10^{5}$ (middle) and $10^{6}$ (bottom) and each column of figures corresponds to Likelihood ratio (left), Wald (middle) and Rao (right) type test statistics. The red line is the cumulative distribution function of $\chi_{1}^{2}$. 

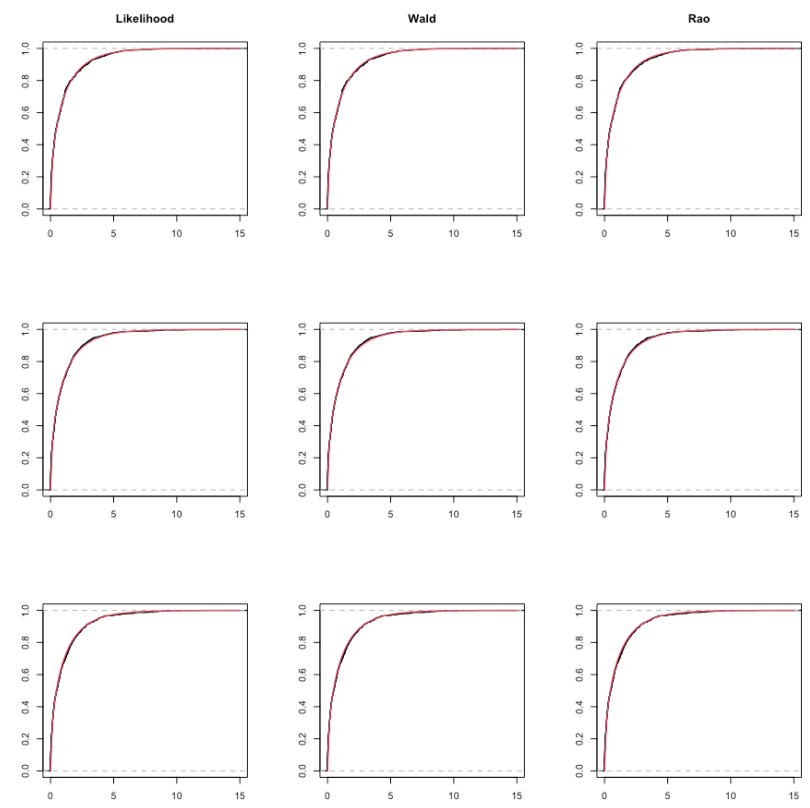

Figure 7. Empirical distributions of the three types of test statistics for $\alpha$ in Case 2. Each row of figures corresponds to the case of $n=10^{4}$ (upper), $10^{5}$ (middle) and $10^{6}$ (bottom) and each column of figures corresponds to Likelihood ratio (left), Wald (middle) and Rao (right) type test statistics. The red line is the cumulative distribution function of $\chi_{1}^{2}$.
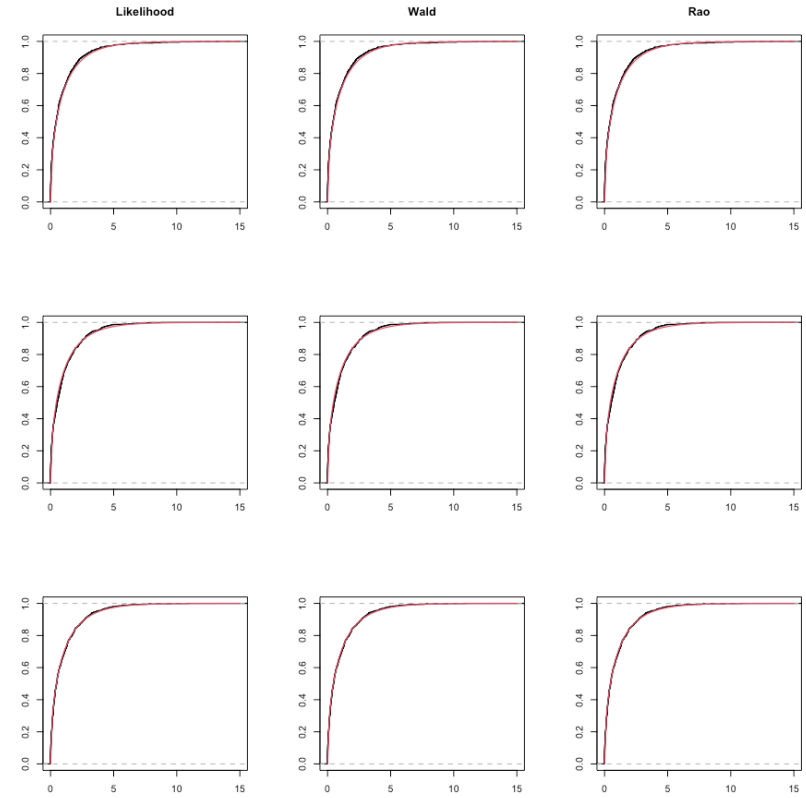

Figure 8. Empirical distributions of the three types of test statistics for $\beta$ in Case 3. Each row of figures corresponds to the case of $n=10^{4}$ (upper), $10^{5}$ (middle) and $10^{6}$ (bottom) and each column of figures corresponds to Likelihood ratio (left), Wald (middle) and Rao (right) type test statistics. The red line is the cumulative distribution function of $\chi_{1}^{2}$. 
TABLE 5. Empirical sizes of the test statistics for $\alpha$ in Case 1.

\begin{tabular}{cc|ccc}
$n$ & $n h_{n}$ & Likelihood & Wald & Rao \\
\hline \hline $10^{4}$ & 21.5 & 0.057 & 0.055 & 0.057 \\
$10^{5}$ & 46.4 & 0.053 & 0.053 & 0.053 \\
$10^{6}$ & 100 & 0.051 & 0.051 & 0.050
\end{tabular}

TABLE 7. Empirical sizes of the test statistics for $\alpha$ in Case 2.

\begin{tabular}{cc|ccc}
$n$ & $n h_{n}$ & Likelihood & Wald & Rao \\
\hline \hline $10^{4}$ & 21.5 & 0.058 & 0.056 & 0.058 \\
$10^{5}$ & 46.4 & 0.045 & 0.043 & 0.046 \\
$10^{6}$ & 100 & 0.049 & 0.049 & 0.049
\end{tabular}

TABLE 6. Empirical sizes of the test statistics for $\beta$ in Case $\mathbf{1}$.

\begin{tabular}{cc|ccc}
$n$ & $n h_{n}$ & Likelihood & Wald & Rao \\
\hline \hline $10^{4}$ & 21.5 & 0.057 & 0.057 & 0.057 \\
$10^{5}$ & 46.4 & 0.053 & 0.053 & 0.053 \\
$10^{6}$ & 100 & 0.053 & 0.053 & 0.053
\end{tabular}

TABLE 8. Empirical sizes of the test statistics for $\beta$ in Case 3.

\begin{tabular}{cc|ccc}
$n$ & $n h_{n}$ & Likelihood & Wald & Rao \\
\hline \hline $10^{4}$ & 21.5 & 0.044 & 0.044 & 0.044 \\
$10^{5}$ & 46.4 & 0.046 & 0.046 & 0.046 \\
$10^{6}$ & 100 & 0.045 & 0.045 & 0.045
\end{tabular}

In order to check consistency of the tests, we treat the three kinds of $\alpha^{*}(1.1,1.01$ and 1.001) in Case 3 and the three kinds of $\beta^{*}(3.0,2.5$ and 2.1) in Case 2. Tables 9 and 10 are simulation results of the empirical powers of the three test statistics. For $\alpha^{*}=1.1,1.01$ and $\beta^{*}=3.0,2.5$, the empirical powers increase and tend to 1 as $n$ increases.

Next, we consider the asymptotic distributions of the three test statistics under local alternatives. The hypothesis tests are defined as

$$
\left\{\begin{array} { l } 
{ H _ { 0 , n } ^ { ( 1 ) } : \alpha = \alpha _ { 0 } = 1 . 0 , } \\
{ H _ { 1 , n } ^ { ( 1 ) } : \alpha = \alpha _ { 1 , n } ^ { * } = \alpha _ { 0 } + \frac { u _ { \alpha } } { \sqrt { n } } , }
\end{array} \quad \left\{\begin{array}{l}
H_{0, n}^{(2)}: \beta=\beta_{0}=2.0, \\
H_{1, n}^{(2)}: \beta=\beta_{1, n}^{*}=\beta_{0}+\frac{u_{\beta}}{\sqrt{n h_{n}}},
\end{array}\right.\right.
$$

where we set $u_{\alpha}=5.0$ and $u_{\beta}=2.0$. In the Ornstein-Uhlenbeck model (3), the invariant distribution is the normal distribution with mean $\beta$ and variance $\frac{\alpha^{2}}{2}$. Therefore we calculate

$$
\begin{gathered}
I_{a}\left(\alpha_{0}, \alpha_{0}\right)=\frac{2.0}{\alpha_{0}^{2}}=2.0, \quad I_{b}\left(\theta_{0}, \theta_{0}\right)=\frac{1.0}{\alpha_{0}^{2}}=1.0, \\
I_{a}\left(\alpha_{0}, \alpha_{0}\right) u_{\alpha}^{2}=50, \quad I_{b}\left(\theta_{0}, \theta_{0}\right) u_{\beta}^{2}=4.0 .
\end{gathered}
$$

Figures 9,12 show the histograms and the empirical distributions of the three test statistics under local alternatives. Theoretically, the asymptotic distributions of the three test statistics for $\alpha$ are $\chi_{1}^{2}(50)$ and those for $\beta$ are $\chi_{1}^{2}(4)$. We see from Figures 9,12 that as $n$ increases, the three test statistics for $\alpha$ and $\beta$ are approximately distributed as $\chi_{1}^{2}(50)$ and $\chi_{1}^{2}(4)$, respectively. In particular, the Likelihood ratio type test statistic for $\alpha$ has good performance.

TABle 9. Empirical sizes of the test statistics for $\alpha$ in Case 3 .

\begin{tabular}{c|c|ccc}
$\alpha^{*}$ & $n$ & Likelihood & Wald & Rao \\
\hline \hline \multirow{3}{*}{1.1} & $10^{4}$ & 1.000 & 1.000 & 1.000 \\
& $10^{5}$ & 1.000 & 1.000 & 1.000 \\
& $10^{6}$ & 1.000 & 1.000 & 1.000 \\
\hline \multirow{3}{*}{1.01} & $10^{4}$ & 0.326 & 0.316 & 0.349 \\
& $10^{5}$ & 0.994 & 0.994 & 0.994 \\
& $10^{6}$ & 1.000 & 1.000 & 1.000 \\
\hline \multirow{3}{*}{1.001} & $10^{4}$ & 0.050 & 0.047 & 0.048 \\
& $10^{5}$ & 0.095 & 0.093 & 0.098 \\
& $10^{6}$ & 0.295 & 0.294 & 0.297
\end{tabular}

TABLE 10. Empirical sizes of the test statistics for $\beta$ in Case 2.

\begin{tabular}{c|c|ccc}
$\beta^{*}$ & $n h_{n}$ & Likelihood & Wald & Rao \\
\hline \hline \multirow{3}{*}{3.0} & 21.5 & 0.994 & 0.994 & 0.994 \\
& 46.4 & 1.000 & 1.000 & 1.000 \\
& 100 & 1.000 & 1.000 & 1.000 \\
\hline \multirow{3}{*}{2.5} & 21.5 & 0.625 & 0.625 & 0.625 \\
& 46.4 & 0.923 & 0.923 & 0.923 \\
& 100 & 0.999 & 0.999 & 0.999 \\
\hline \multirow{3}{*}{2.1} & 21.5 & 0.091 & 0.091 & 0.091 \\
& 46.4 & 0.098 & 0.098 & 0.098 \\
& 100 & 0.154 & 0.154 & 0.154
\end{tabular}



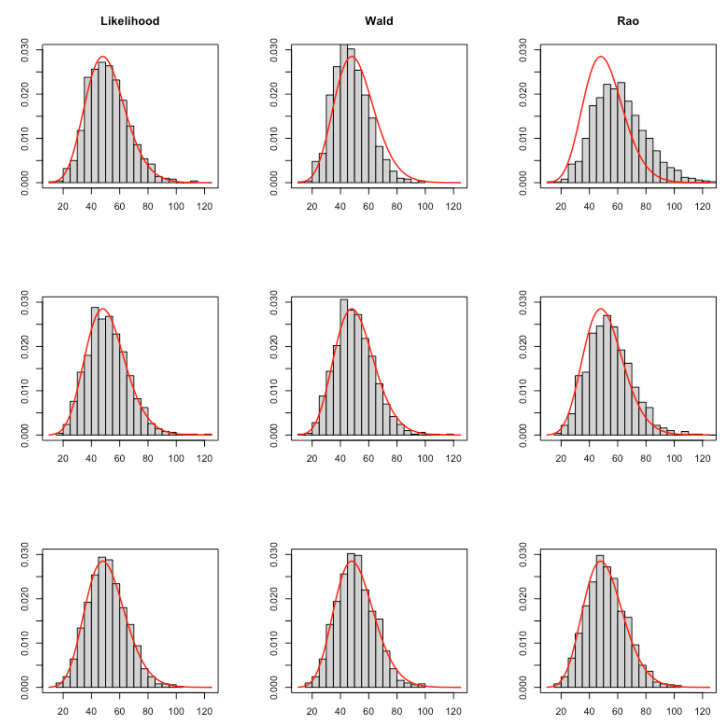

Figure 9. Histograms of the three types of test statistics for $\alpha$ under $H_{1, n}^{(1)}$. Each row of figures corresponds to the case of $n=10^{4}$ (upper), $10^{5}$ (middle) and $10^{6}$ (bottom) and each column of figures corresponds to Likelihood ratio (left), Wald (middle) and Rao (right) type test statistics. The red line is the probability density function of $\chi_{2}^{2}(50)$.
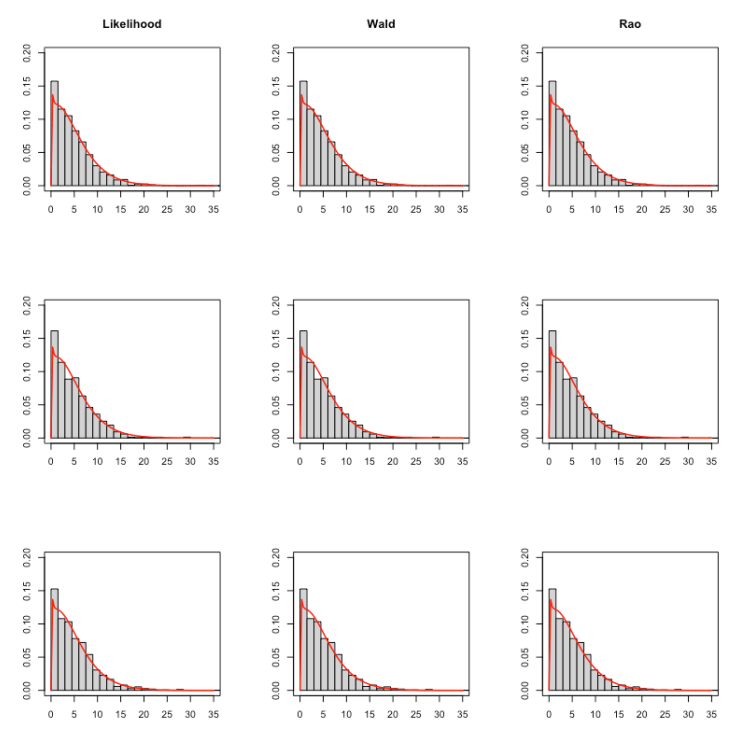

Figure 10. Histograms of the three types of test statistics for $\beta$ under $H_{1, n}^{(2)}$. Each row of figures corresponds to the case of $n=10^{4}$ (upper), $10^{5}$ (middle) and $10^{6}$ (bottom) and each column of figures corresponds to Likelihood ratio (left), Wald (middle) and Rao (right) type test statistics. The red line is the probability density function of $\chi_{2}^{2}(4)$. 

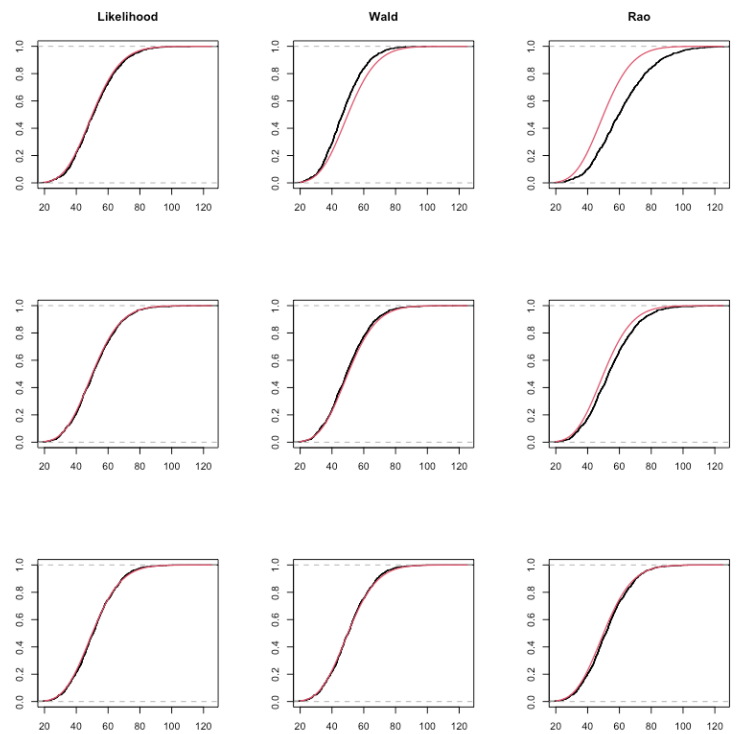

FIGURE 11. Empirical distributions of the three types of test statistics for $\alpha$ under $H_{1, n}^{(1)}$. Each row of figures corresponds to the case of $n=10^{4}$ (upper), $10^{5}$ (middle) and $10^{6}$ (bottom) and each column of figures corresponds to Likelihood ratio (left), Wald (middle) and Rao (right) type test statistics. The red line is the cumulative distribution function of $\chi_{2}^{2}(50)$.
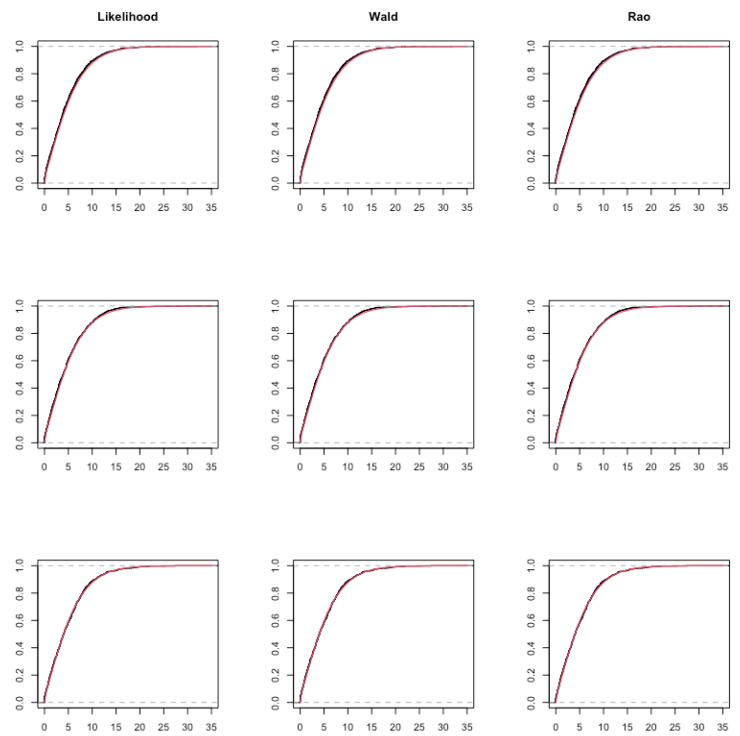

FIGURE 12. Empirical distributions of the three types of test statistics for $\beta$ under $H_{1, n}^{(1)}$. Each row of figures corresponds to the case of $n=10^{4}$ (upper), $10^{5}$ (middle) and $10^{6}$ (bottom) and each column of figures corresponds to Likelihood ratio (left), Wald (middle) and Rao (right) type test statistics. The red line is the cumulative distribution function of $\chi_{2}^{2}(4)$. 


\subsection{Model 2.}

Next, we consider a 1-dimensional diffusion process satisfying the following stochastic differential equation which is more complex than model 1 :

$$
\left\{\begin{array}{l}
d X_{t}=-\beta_{1}\left(X_{t}-\beta_{2}\right) d t+\left(\alpha_{1}+\frac{\alpha_{2}}{1+X_{t}^{2}}+\alpha_{3} \cos ^{2} X_{t}\right) d W_{t}, \\
X_{0}=1.0
\end{array}\right.
$$

We deal with the following tests:

$$
\left\{\begin{array}{l}
H_{0}^{(1)}:\left(\alpha_{1}, \alpha_{2}\right)=(1.0,1.0), \quad\left\{\begin{array}{l}
H_{0}^{(2)}:\left(\beta_{1}, \beta_{2}\right)=(2.0,2.0), \\
H_{1}^{(1)}: \operatorname{not} H_{0}^{(1)},
\end{array} \quad \text { not } H_{0}^{(2)} .\right.
\end{array}\right.
$$

We set the true parameter $\alpha_{3}^{*}=0.5$ and choice the true parameter $\left(\alpha_{1}^{*}, \alpha_{2}^{*}, \beta_{1}^{*}, \beta_{2}^{*}\right)$ from $\{(1.0,1.0,2.0,2.0)$, $(1.0,1.0,2.5,2.5),(1.05,1.05,2.0,2.0),(1.05,1.05,2.5,2.5)\}$, which corresponds to the true parameter of Cases 1-4 described in Section 4.1 respectively. Each test is rejected when the realization of each test statistics is greater than $\chi_{2,0.05}^{2}$, and the other simulation settings are the same as in the model (3) of Section 4.1.

Tables 11 14 show the number of counts of Cases 1-4 selected by the tests (6), where the true parameter $\left(\alpha_{1}^{*}, \alpha_{2}^{*}, \beta_{1}^{*}, \beta_{2}^{*}\right)$ corresponds to each case. In all of the tables, the true case is most often selected as $n$ increases. However, the Rao type test statistic does not have good performance in Cases 2 and 4 when $n=10^{4}$. Figures 1320 are simulation results of the histograms and empirical distributions of the three types of test statistics in Theorem 1. Theoretically, these test statistics converge in distribution to $\chi_{2}^{2}$. Tables 1518 show the empirical sizes of the three types of test statistics when null hypothesis is true. From these figures and tables, the Likelihood ratio type test statistic has the best behavior of the three types of test statistics.

TABle 11. Results of test statistics in Case 1: $\left(\alpha_{1}^{*}, \alpha_{2}^{*}, \beta_{1}^{*}, \beta_{2}^{*}\right)=(1.0,1.0,2.0,2.0)$.

\begin{tabular}{ccc|c|cccc}
$n$ & $h_{n}$ & $n h_{n}$ & Test type & Case 1 & Case 2 & Case 3 & Case 4 \\
\hline \hline \multirow{3}{*}{$10^{4}$} & \multirow{2}{*}{$2.15 \times 10^{-3}$} & \multirow{2}{*}{21.5} & Likelihood & 896 & 53 & 47 & 4 \\
& & Wald & 878 & 68 & 48 & 6 \\
& & Rao & 894 & 53 & 49 & 4 \\
\hline \multirow{3}{*}{$10^{5}$} & \multirow{3}{*}{$4.64 \times 10^{-4}$} & \multirow{2}{*}{46.4} & Likelihood & 896 & 55 & 47 & 2 \\
& & & Wald & 884 & 68 & 46 & 2 \\
& & Rao & 895 & 56 & 48 & 1 \\
\hline \multirow{3}{*}{$10^{6}$} & \multirow{3}{*}{$1.00 \times 10^{-4}$} & \multirow{2}{*}{100} & Likelihood & 905 & 41 & 51 & 3 \\
& & & Wald & 902 & 44 & 51 & 3 \\
& & Rao & 914 & 32 & 54 & 0
\end{tabular}

TABle 12. Results of test statistics in Case 2: $\left(\alpha_{1}^{*}, \alpha_{2}^{*}, \beta_{1}^{*}, \beta_{2}^{*}\right)=(1.0,1.0,2.5,2.5)$.

\begin{tabular}{ccc|c|cccc}
$n$ & $h_{n}$ & $n h_{n}$ & Test type & Case 1 & Case 2 & Case 3 & Case 4 \\
\hline \hline \multirow{3}{*}{$10^{4}$} & \multirow{3}{*}{$2.15 \times 10^{-3}$} & \multirow{2}{*}{21.5} & Likelihood & 55 & 888 & 5 & 52 \\
& & Wald & 37 & 904 & 4 & 55 \\
& & Rao & 325 & 613 & 24 & 38 \\
\hline \multirow{3}{*}{$10^{5}$} & \multirow{3}{*}{$4.64 \times 10^{-4}$} & \multirow{2}{*}{46.4} & Likelihood & 0 & 947 & 0 & 53 \\
& & & Wald & 0 & 944 & 0 & 56 \\
& & Rao & 11 & 936 & 0 & 53 \\
\hline \multirow{3}{*}{$10^{6}$} & \multirow{3}{*}{$1.00 \times 10^{-4}$} & \multirow{2}{*}{100} & Likelihood & 0 & 949 & 0 & 51 \\
& & & Wald & 0 & 949 & 0 & 51 \\
& & Rao & 0 & 946 & 0 & 54
\end{tabular}


TABLE 13. Results of test statistics in Case 3: $\left(\alpha_{1}^{*}, \alpha_{2}^{*}, \beta_{1}^{*}, \beta_{2}^{*}\right)=(1.05,1.05,2.0,2.0)$.

\begin{tabular}{ccc|c|cccc}
$n$ & $h_{n}$ & $n h_{n}$ & Test type & Case 1 & Case 2 & Case 3 & Case 4 \\
\hline \hline \multirow{3}{*}{$10^{4}$} & \multirow{3}{*}{$2.15 \times 10^{-3}$} & \multirow{3}{*}{21.5} & Likelihood & 12 & 2 & 926 & 60 \\
& & Wald & 13 & 2 & 908 & 77 \\
& & Rao & 9 & 4 & 923 & 64 \\
\hline \multirow{3}{*}{$10^{5}$} & \multirow{3}{*}{$4.64 \times 10^{-4}$} & \multirow{3}{*}{46.4} & Likelihood & 0 & 0 & 955 & 45 \\
& & & Wald & 0 & 0 & 946 & 54 \\
& & Rao & 0 & 0 & 954 & 46 \\
\hline \multirow{3}{*}{$10^{6}$} & \multirow{3}{*}{$1.00 \times 10^{-4}$} & \multirow{2}{*}{100} & Likelihood & 0 & 0 & 951 & 49 \\
& & & Wald & 0 & 0 & 946 & 54 \\
& & & Rao & 0 & 0 & 956 & 44
\end{tabular}

TABLE 14. Results of test statistics in Case $4:\left(\alpha_{1}^{*}, \alpha_{2}^{*}, \beta_{1}^{*}, \beta_{2}^{*}\right)=(1.05,1.05,2.5,2.5)$.

\begin{tabular}{ccc|c|cccc}
$n$ & $h_{n}$ & $n h_{n}$ & Test type & Case 1 & Case 2 & Case 3 & Case 4 \\
\hline \hline \multirow{3}{*}{$10^{4}$} & \multirow{3}{*}{$2.15 \times 10^{-3}$} & \multirow{2}{*}{21.5} & Likelihood & 2 & 76 & 71 & 851 \\
& & Wald & 1 & 91 & 44 & 864 \\
& & Rao & 21 & 47 & 412 & 520 \\
\hline \multirow{3}{*}{$10^{5}$} & \multirow{3}{*}{$4.64 \times 10^{-4}$} & \multirow{2}{*}{46.4} & Likelihood & 0 & 0 & 0 & 1000 \\
& & & Wald & 0 & 0 & 0 & 1000 \\
& & Rao & 0 & 0 & 12 & 988 \\
\hline \multirow{3}{*}{$10^{6}$} & \multirow{3}{*}{$1.00 \times 10^{-4}$} & \multirow{2}{*}{100} & Likelihood & 0 & 0 & 0 & 1000 \\
& & & Wald & 0 & 0 & 0 & 1000 \\
& & & Rao & 0 & 0 & 0 & 1000
\end{tabular}
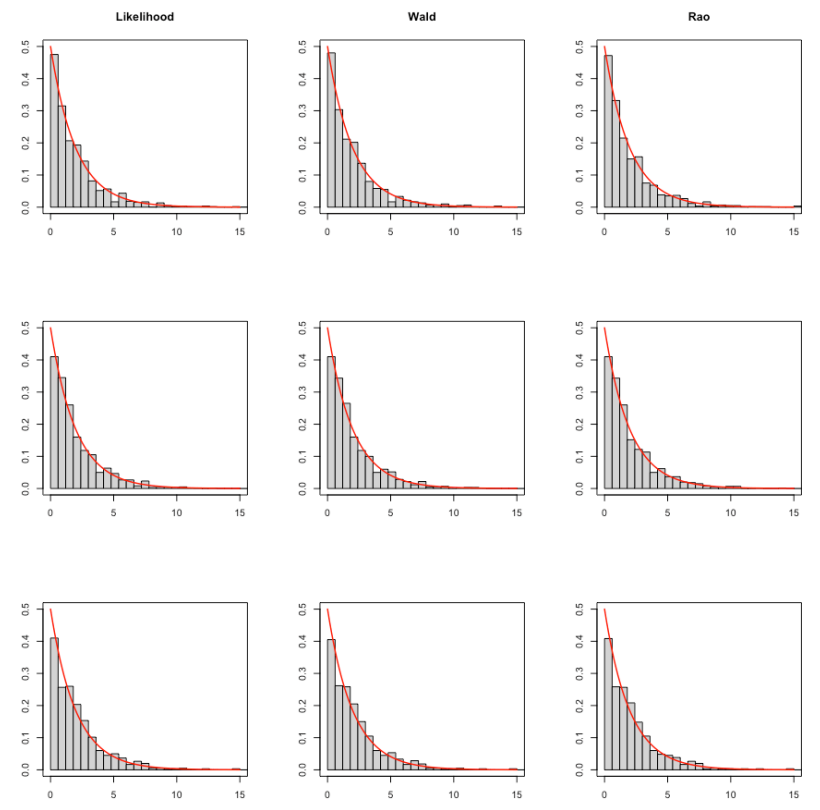

Figure 13. Histograms of the three types of test statistics for $\alpha$ in Case 1. Each row of figures corresponds to the case of $n=10^{4}$ (upper), $10^{5}$ (middle) and $10^{6}$ (bottom) and each column of figures corresponds to Likelihood ratio (left), Wald (middle) and Rao (right) type test statistics. The red line is the probability density function of $\chi_{2}^{2}$. 

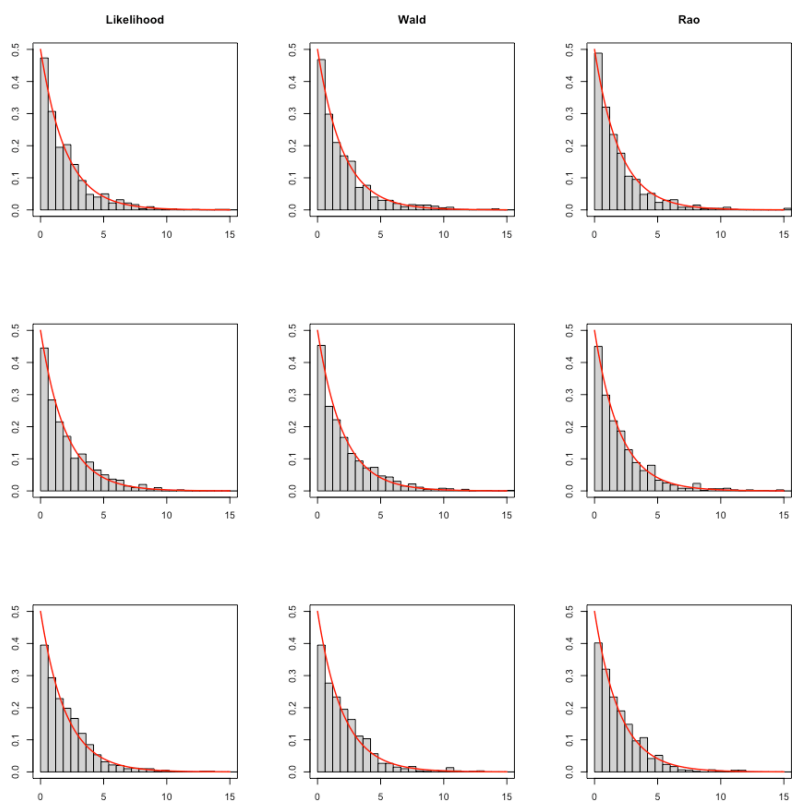

Figure 14. Histograms of the three types of test statistics for $\beta$ in Case 1. Each row of figures corresponds to the case of $n=10^{4}$ (upper), $10^{5}$ (middle) and $10^{6}$ (bottom) and each column of figures corresponds to Likelihood ratio (left), Wald (middle) and Rao (right) type test statistics. The red line is the probability density function of $\chi_{2}^{2}$.
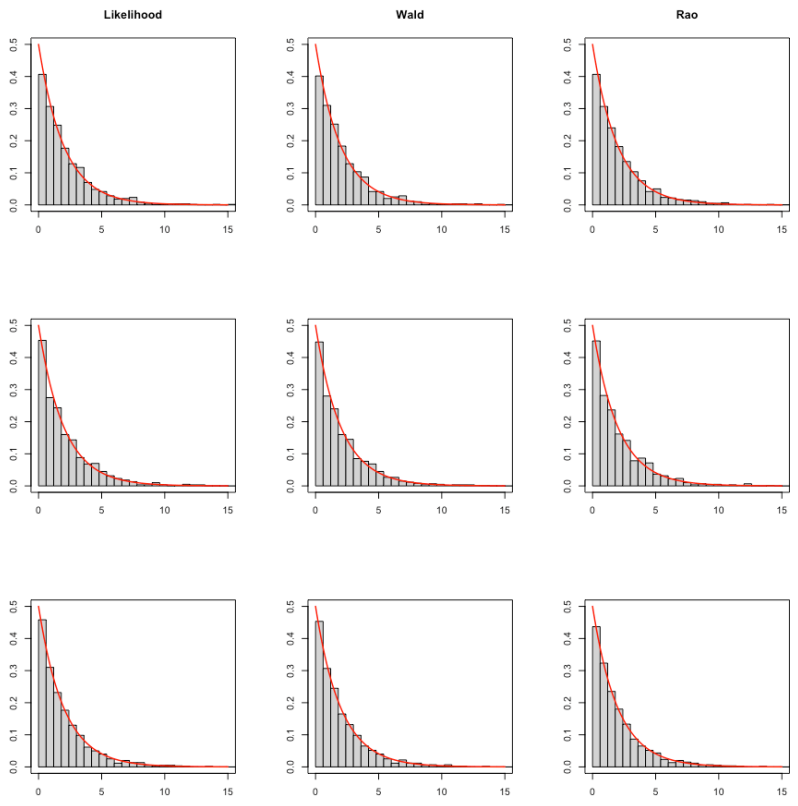

Figure 15. Histograms of the three types of test statistics for $\alpha$ in Case 2. Each row of figures corresponds to the case of $n=10^{4}$ (upper), $10^{5}$ (middle) and $10^{6}$ (bottom) and each column of figures corresponds to Likelihood ratio (left), Wald (middle) and Rao (right) type test statistics. The red line is the probability density function of $\chi_{2}^{2}$. 

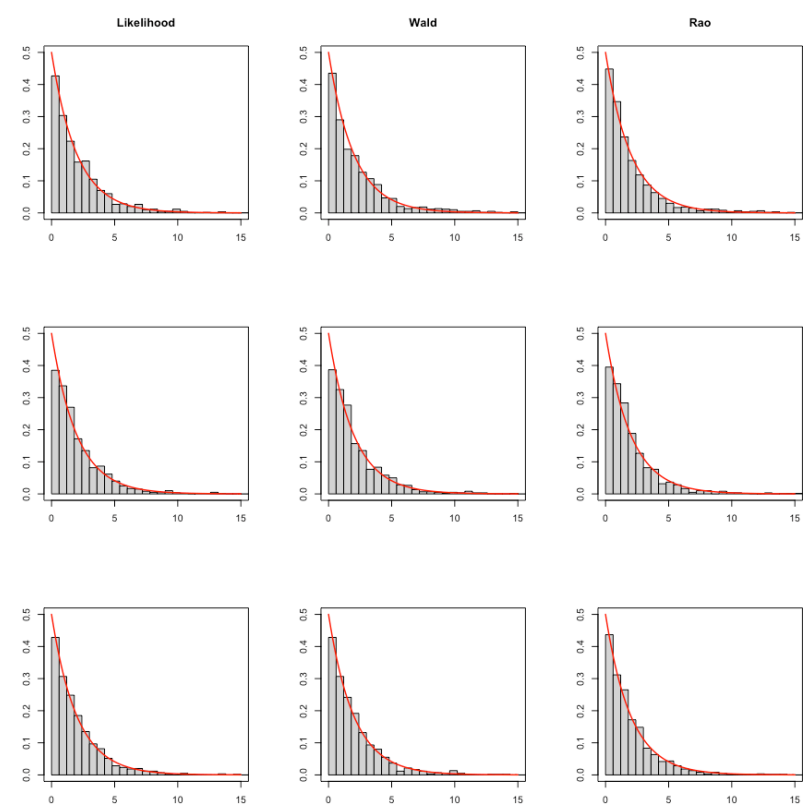

Figure 16. Histograms of the three types of test statistics for $\beta$ in Case 3. Each row of figures corresponds to the case of $n=10^{4}$ (upper), $10^{5}$ (middle) and $10^{6}$ (bottom) and each column of figures corresponds to Likelihood ratio (left), Wald (middle) and Rao (right) type test statistics. The red line is the probability density function of $\chi_{2}^{2}$.
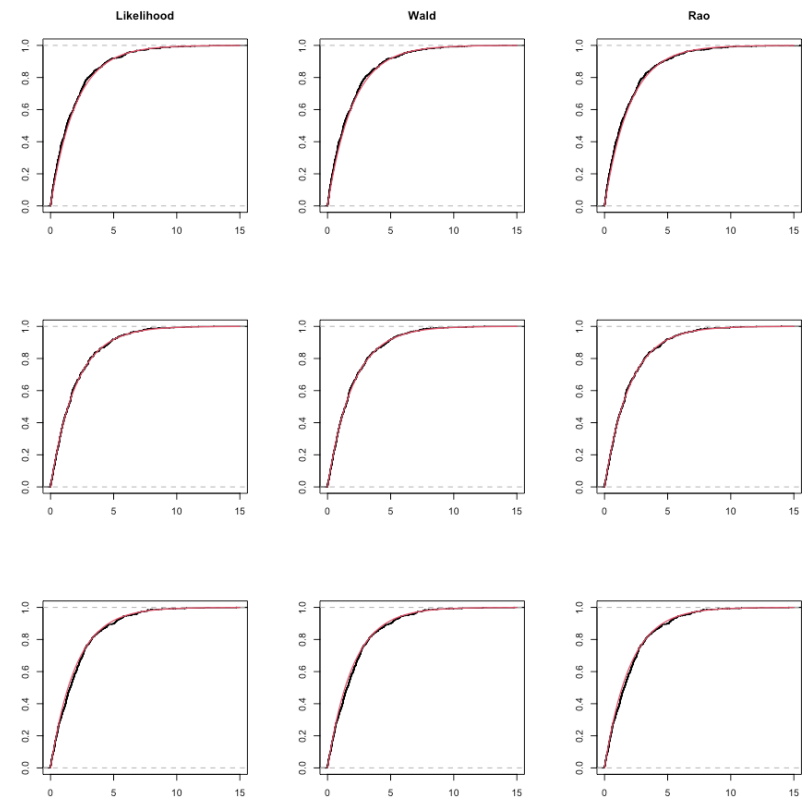

Figure 17. Empirical distributions of the three types of test statistics for $\alpha$ in Case 1. Each row of figures corresponds to the case of $n=10^{4}$ (upper), $10^{5}$ (middle) and $10^{6}$ (bottom) and each column of figures corresponds to Likelihood ratio (left), Wald (middle) and Rao (right) type test statistics. The red line is the cumulative distribution function of $\chi_{2}^{2}$. 

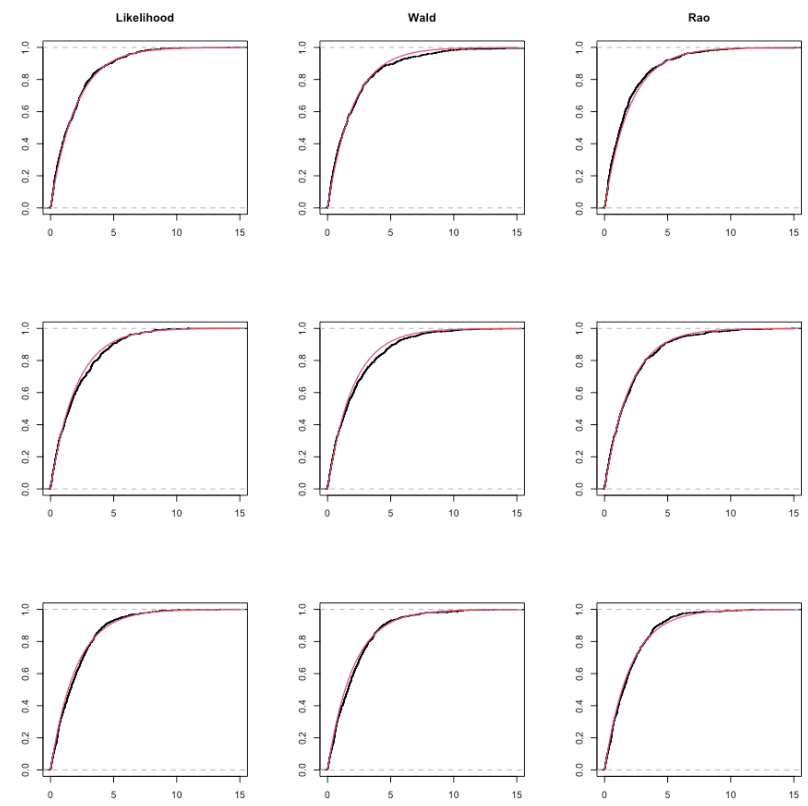

Figure 18. Empirical distributions of the three types of test statistics for $\beta$ in Case 1. Each row of figures corresponds to the case of $n=10^{4}$ (upper), $10^{5}$ (middle) and $10^{6}$ (bottom) and each column of figures corresponds to Likelihood ratio (left), Wald (middle) and Rao (right) type test statistics. The red line is the cumulative distribution function of $\chi_{2}^{2}$.
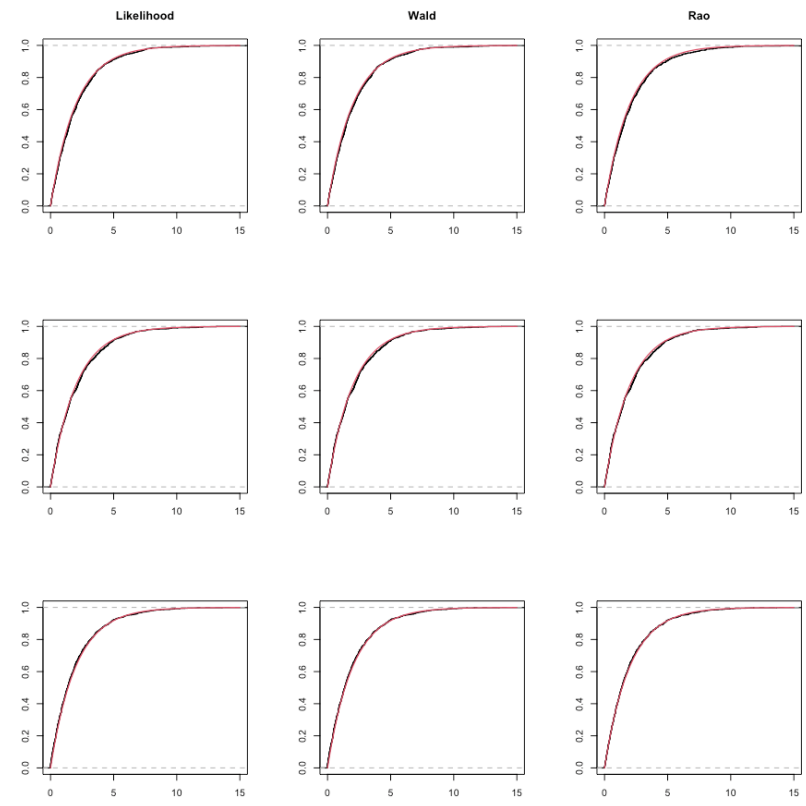

Figure 19. Empirical distributions of the three types of test statistics for $\alpha$ in Case 2. Each row of figures corresponds to the case of $n=10^{4}$ (upper), $10^{5}$ (middle) and $10^{6}$ (bottom) and each column of figures corresponds to Likelihood ratio (left), Wald (middle) and Rao (right) type test statistics. The red line is the cumulative distribution function of $\chi_{2}^{2}$. 

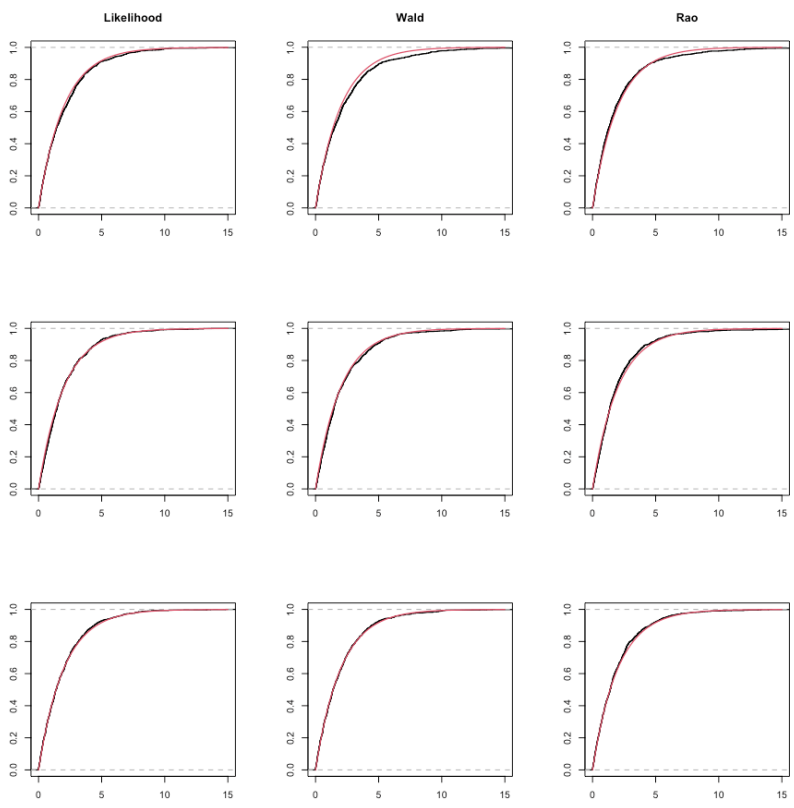

Figure 20. Empirical distributions of the three types of test statistics for $\beta$ in Case 3. Each row of figures corresponds to the case of $n=10^{4}$ (upper), $10^{5}$ (middle) and $10^{6}$ (bottom) and each column of figures corresponds to Likelihood ratio (left), Wald (middle) and Rao (right) type test statistics. The red line is the cumulative distribution function of $\chi_{2}^{2}$.

TABLE 15. Empirical sizes of the test statistics for $\alpha$ in Case 1.

\begin{tabular}{cc|ccc}
$n$ & $n h_{n}$ & Likelihood & Wald & Rao \\
\hline \hline $10^{4}$ & 21.5 & 0.051 & 0.054 & 0.053 \\
$10^{5}$ & 46.4 & 0.049 & 0.048 & 0.049 \\
$10^{6}$ & 100 & 0.054 & 0.054 & 0.054
\end{tabular}

TABLE 17. Empirical sizes of the test statistics for $\alpha$ in Case 2.

\begin{tabular}{cc|ccc}
$n$ & $n h_{n}$ & Likelihood & Wald & Rao \\
\hline \hline $10^{4}$ & 21.5 & 0.057 & 0.059 & 0.062 \\
$10^{5}$ & 46.4 & 0.053 & 0.056 & 0.053 \\
$10^{6}$ & 100 & 0.051 & 0.051 & 0.054
\end{tabular}

TABLE 16. Empirical sizes of the test statistics for $\beta$ in Case 1.

\begin{tabular}{cc|ccc}
$n$ & $n h_{n}$ & Likelihood & Wald & Rao \\
\hline \hline $10^{4}$ & 21.5 & 0.057 & 0.074 & 0.057 \\
$10^{5}$ & 46.4 & 0.057 & 0.070 & 0.057 \\
$10^{6}$ & 100 & 0.044 & 0.047 & 0.032
\end{tabular}

TABLE 18. Empirical sizes of the test statistics for $\beta$ in Case 3 .

\begin{tabular}{cc|ccc}
$n$ & $n h_{n}$ & Likelihood & Wald & Rao \\
\hline \hline $10^{4}$ & 21.5 & 0.062 & 0.079 & 0.068 \\
$10^{5}$ & 46.4 & 0.045 & 0.054 & 0.046 \\
$10^{6}$ & 100 & 0.049 & 0.054 & 0.044
\end{tabular}

Next, in order to check consistency of the tests, we treat the three kinds of $\left(\alpha_{1}^{*}, \alpha_{2}^{*}\right)((1.05,1.0),(1.0,1.05)$ and $(1.05,1.05))$ in Case 3 and the three kinds of $\left(\beta_{1}^{*}, \beta_{2}^{*}\right)((2.5,2.0),(2.0,2.5)$ and $(2.5,2.5))$ in Case 2. Tables 19 and 20 show the empirical powers of the three types of test statistics. In table 19 , when $n \leq 10^{5}$, since the asymptotic behavior of the estimator of $\alpha_{1}$ is stable compared with that of $\alpha_{2}$, the empirical power in the case of $\left(\alpha_{1}^{*}, \alpha_{2}^{*}\right)=(1.05,1.0)$ is greater than that in the case of $\left(\alpha_{1}^{*}, \alpha_{2}^{*}\right)=(1.0,1.05)$. In Table 20 , when $n h_{n} \leq 100$, the empirical power in the case of $\left(\beta_{1}^{*}, \beta_{2}^{*}\right)=(2.0,2.5)$ is greater than that in the case of $\left(\beta_{1}^{*}, \beta_{2}^{*}\right)=(2.5,2.0)$ because the asymptotic performance of the estimator of $\beta_{2}$ is better than that of $\beta_{1}$. 
TABLE 19. Empirical powers of the three types of test statistics for $\alpha$ in Case 3.

\begin{tabular}{c|c|ccc}
$\left(\alpha_{1}^{*}, \alpha_{2}^{*}\right)$ & $n$ & Likelihood & Wald & Rao \\
\hline \hline & $10^{4}$ & 0.898 & 0.892 & 0.907 \\
$(1.05,1.0)$ & $10^{5}$ & 1.000 & 1.000 & 1.000 \\
& $10^{6}$ & 1.000 & 1.000 & 1.000 \\
\hline & $10^{4}$ & 0.164 & 0.149 & 0.185 \\
$(1.0,1.05)$ & $10^{5}$ & 0.918 & 0.915 & 0.921 \\
& $10^{6}$ & 1.000 & 1.000 & 1.000 \\
\hline & $10^{4}$ & 0.986 & 0.985 & 0.987 \\
$(1.05,1.05)$ & $10^{5}$ & 1.000 & 1.000 & 1.000 \\
& $10^{6}$ & 1.000 & 1.000 & 1.000
\end{tabular}

TABLE 20. Empirical powers of the three types of test statistics for $\beta$ in Case 2.

\begin{tabular}{c|c|ccc}
$\left(\beta_{1}^{*}, \beta_{2}^{*}\right)$ & $n h_{n}$ & Likelihood & Wald & Rao \\
\hline \hline & 21.5 & 0.142 & 0.200 & 0.075 \\
$(2.5,2.0)$ & 46.4 & 0.207 & 0.261 & 0.141 \\
& 100 & 0.401 & 0.427 & 0.342 \\
\hline & 21.5 & 0.861 & 0.848 & 0.780 \\
$(2.0,2.5)$ & 46.4 & 0.997 & 0.994 & 0.984 \\
& 100 & 1.000 & 1.000 & 1.000 \\
\hline & 21.5 & 0.940 & 0.959 & 0.651 \\
$(2.5,2.5)$ & 46.4 & 1.000 & 1.000 & 0.989 \\
& 100 & 1.000 & 1.000 & 1.000
\end{tabular}

\section{Proofs}

Let

$$
I_{a}\left(\alpha_{0} ; \alpha_{0}\right)=\left(\begin{array}{cc}
I_{a, 1}\left(\alpha_{0} ; \alpha_{0}\right) & I_{a, 2}\left(\alpha_{0} ; \alpha_{0}\right) \\
I_{a, 2}^{\top}\left(\alpha_{0} ; \alpha_{0}\right) & I_{a, 3}\left(\alpha_{0} ; \alpha_{0}\right)
\end{array}\right), \quad H_{1}=\left(\begin{array}{cc}
0 & 0 \\
0 & I_{a, 3}^{-1}\left(\alpha_{0} ; \alpha_{0}\right)
\end{array}\right)
$$

where $I_{a, 1}\left(\alpha_{0} ; \alpha_{0}\right), I_{a, 2}\left(\alpha_{0} ; \alpha_{0}\right), I_{a, 3}\left(\alpha_{0} ; \alpha_{0}\right)$ are the $\left(r_{1} \times r_{1}\right),\left(r_{1} \times\left(p_{1}-r_{1}\right)\right)$ and $\left(\left(p_{1}-r_{1}\right) \times\left(p_{1}-r_{1}\right)\right)$ matrices, respectively. Set

$$
I_{b}\left(\theta_{0} ; \theta_{0}\right)=\left(\begin{array}{cc}
I_{b, 1}\left(\theta_{0} ; \theta_{0}\right) & I_{b, 2}\left(\theta_{0} ; \theta_{0}\right) \\
I_{b, 2}^{\dagger}\left(\theta_{0} ; \theta_{0}\right) & I_{b, 3}\left(\theta_{0} ; \theta_{0}\right)
\end{array}\right), \quad H_{2}=\left(\begin{array}{cc}
0 & 0 \\
0 & I_{b, 3}^{-1}\left(\theta_{0} ; \theta_{0}\right)
\end{array}\right),
$$

where $I_{b, 1}\left(\theta_{0} ; \theta_{0}\right), I_{b, 2}\left(\theta_{0} ; \theta_{0}\right), I_{b, 3}\left(\theta_{0} ; \theta_{0}\right)$ are the $\left(r_{2} \times r_{2}\right),\left(r_{2} \times\left(p_{2}-r_{2}\right)\right)$ and $\left(\left(p_{2}-r_{2}\right) \times\left(p_{2}-r_{2}\right)\right)$ matrices, respectively. Moreover, $Y_{1}$ and $Y_{2}$ are the normal random variables with mean 0 and covariance matrices $I_{a}\left(\alpha_{0} ; \alpha_{0}\right)$ and $I_{b}\left(\theta_{0} ; \theta_{0}\right)$, respectively, that is, $Y_{1} \sim N\left(0, I_{a}\left(\alpha_{0} ; \alpha_{0}\right)\right)$ and $Y_{2} \sim N\left(0, I_{b}\left(\theta_{0} ; \theta_{0}\right)\right)$.

In order to proof Theorem 1 , we need the following lemma.

Lemma 1 Assume A1-A7. If $h_{n} \rightarrow 0, n h_{n} \rightarrow \infty$ and $n h_{n}^{2} \rightarrow 0$, then

(i) $\sqrt{n}\left(\hat{\alpha}_{n}-\tilde{\alpha}_{n}\right) \stackrel{d}{\rightarrow}\left(I_{a}^{-1}\left(\alpha_{0} ; \alpha_{0}\right)-H_{1}\right) Y_{1} \quad$ (under $\left.H_{0}^{(1)}\right)$,

(ii) $\sqrt{n h_{n}}\left(\hat{\beta}_{n}-\tilde{\beta}_{n}\right) \stackrel{d}{\rightarrow}\left(I_{b}^{-1}\left(\theta_{0} ; \theta_{0}\right)-H_{2}\right) Y_{2} \quad$ (under $\left.H_{0}^{(2)}\right)$.

Proof. (i) First of all, we will show that

$$
H_{1} \frac{1}{\sqrt{n}} \partial_{\alpha} U_{n}^{(1)}\left(\alpha_{0}\right)=\sqrt{n}\left(\tilde{\alpha}_{n}-\alpha_{0}\right)+o_{P}(1) \quad\left(\text { under } H_{0}^{(1)}\right) .
$$

From Taylor's theorem,

$$
\frac{1}{\sqrt{n}} \partial_{\alpha} U_{n}^{(1)}\left(\tilde{\alpha}_{n}\right)-\frac{1}{\sqrt{n}} \partial_{\alpha} U_{n}^{(1)}\left(\alpha_{0}\right)=\tilde{I}_{a, n}\left(\tilde{\alpha}_{n}, \alpha_{0}\right) \sqrt{n}\left(\tilde{\alpha}_{n}-\alpha_{0}\right),
$$

where

$$
\tilde{I}_{a, n}\left(\tilde{\alpha}_{n}, \alpha_{0}\right):=\int_{0}^{1} \frac{1}{n} \partial_{\alpha}^{2} U_{n}^{(1)}\left(\alpha_{0}+u\left(\tilde{\alpha}_{n}-\alpha_{0}\right)\right) d u .
$$

Noting that $H_{1} \partial_{\alpha} U_{n}^{(1)}\left(\tilde{\alpha}_{n}\right)=0$, we obtain

$$
H_{1} \frac{1}{\sqrt{n}} \partial_{\alpha} U_{n}^{(1)}\left(\alpha_{0}\right)=-H_{1} \tilde{I}_{a, n}\left(\tilde{\alpha}_{n}, \alpha_{0}\right) \sqrt{n}\left(\tilde{\alpha}_{n}-\alpha_{0}\right) .
$$


On the other hand, we can show

$$
\begin{array}{rr}
-\tilde{I}_{a, n}\left(\tilde{\alpha}_{n}, \alpha_{0}\right) \stackrel{P}{\rightarrow} I_{a}\left(\alpha_{0} ; \alpha_{0}\right) & \left(\text { under } H_{0}^{(1)}\right), \\
\frac{1}{\sqrt{n}} \partial_{\alpha} U_{n}^{(1)}\left(\alpha_{0}\right) \stackrel{d}{\rightarrow} Y_{1} & \left(\text { under } H_{0}^{(1)}\right), \\
H_{1} \frac{1}{\sqrt{n}} \partial_{\alpha} U_{n}^{(1)}\left(\alpha_{0}\right) \stackrel{d}{\rightarrow} H_{1} Y_{1} & \left(\text { under } H_{0}^{(1)}\right) .
\end{array}
$$

We set $\tilde{\alpha}_{n}^{\left(p_{1}-r_{1}\right)}=\left(\tilde{\alpha}_{n, r_{1}+1}-\alpha_{0, r_{1}+1}, \ldots, \tilde{\alpha}_{n, p_{1}}-\alpha_{0, p_{1}}\right)$. Noting that the first $r_{1}$ components of $\sqrt{n}\left(\tilde{\alpha}_{n}-\alpha_{0}\right)$ are all 0 , one has that under $H_{0}^{(1)}$,

$$
\begin{aligned}
H_{1} \tilde{I}_{a, n}\left(\alpha_{0}, \alpha_{0}\right) \sqrt{n}\left(\tilde{\alpha}_{n}-\alpha_{0}\right) & =\left(\begin{array}{cc}
0 & 0 \\
I_{a, 3}^{-1} \tilde{I}_{a, n, 2}^{T}\left(\alpha_{0}, \alpha_{0}\right) & I_{a, 3}^{-1} \tilde{I}_{a, n, 3}\left(\alpha_{0}, \alpha_{0}\right)
\end{array}\right)\left(\begin{array}{c}
0 \\
\sqrt{n} \tilde{\alpha}_{n}^{\left(p_{1}-r_{1}\right)}
\end{array}\right) \\
& =\left(\begin{array}{c}
0 \\
I_{a, 3}^{-1} \tilde{I}_{a, n, 3}\left(\alpha_{0}, \alpha_{0}\right) \sqrt{n} \tilde{\alpha}_{n}^{\left(p_{1}-r_{1}\right)}
\end{array}\right), \\
H_{1} I_{a}\left(\alpha_{0} ; \alpha_{0}\right) \sqrt{n}\left(\tilde{\alpha}_{n}-\alpha_{0}\right) & =\left(\begin{array}{cc}
0 & 0 \\
I_{a, 3}^{-1} I_{a, 2}^{T}\left(\alpha_{0} ; \alpha_{0}\right) & E_{p_{1}-r_{1}}
\end{array}\right)\left(\begin{array}{c}
0 \\
\sqrt{n} \tilde{\alpha}_{n}^{\left(p_{1}-r_{1}\right)}
\end{array}\right)=\sqrt{n}\left(\tilde{\alpha}_{n}-\alpha_{0}\right),
\end{aligned}
$$

where $E_{p}$ is the $(p \times p)$ identity matrix. The above equations $\left.\left.[9], 10\right], 12\right]$ and $[13)$ implies that under $H_{0}^{(1)}$,

$$
\sqrt{n} \tilde{\alpha}_{n}^{\left(p_{1}-r_{1}\right)}=O_{P}(1)
$$

Since the first $r_{1}$ components of $\sqrt{n}\left(\tilde{\alpha}_{n}-\alpha_{0}\right)$ are all 0 , it follows that

$$
\sqrt{n}\left(\tilde{\alpha}_{n}-\alpha_{0}\right)=O_{P}(1) \quad\left(\text { under } H_{0}^{(1)}\right) \text {. }
$$

Hence, by (9), 10), (14) and $(15)$, we have that under $H_{0}^{(1)}$,

$$
\begin{aligned}
H_{1} \frac{1}{\sqrt{n}} \partial_{\alpha} U_{n}^{(1)}\left(\alpha_{0}\right) & =H_{1}\left(I_{a}\left(\alpha_{0} ; \alpha_{0}\right)+o_{P}(1)\right) \sqrt{n}\left(\tilde{\alpha}_{n}-\alpha_{0}\right) \\
& =H_{1} I_{a}\left(\alpha_{0} ; \alpha_{0}\right) \sqrt{n}\left(\tilde{\alpha}_{n}-\alpha_{0}\right)+o_{P}(1) \\
& =\sqrt{n}\left(\tilde{\alpha}_{n}-\alpha_{0}\right)+o_{P}(1) .
\end{aligned}
$$

This accords with (7). Next, it follows from (8), 10, (15), (7) and (11), that

$$
\begin{aligned}
\frac{1}{\sqrt{n}} \partial_{\alpha} U_{n}^{(1)}\left(\tilde{\alpha}_{n}\right) & =\frac{1}{\sqrt{n}} \partial_{\alpha} U_{n}^{(1)}\left(\alpha_{0}\right)+\tilde{I}_{a, n}\left(\tilde{\alpha}_{n}, \alpha_{0}\right) \sqrt{n}\left(\tilde{\alpha}_{n}-\alpha_{0}\right) \\
& =\frac{1}{\sqrt{n}} \partial_{\alpha} U_{n}^{(1)}\left(\alpha_{0}\right)-I_{a}\left(\alpha_{0} ; \alpha_{0}\right) \sqrt{n}\left(\tilde{\alpha}_{n}-\alpha_{0}\right)+o_{P}(1) \\
& =\frac{1}{\sqrt{n}} \partial_{\beta} U_{n}^{(1)}\left(\alpha_{0}\right)-I_{a}\left(\alpha_{0} ; \alpha_{0}\right) H_{1} \frac{1}{\sqrt{n}} \partial_{\alpha} U_{n}^{(1)}\left(\alpha_{0}\right)+o_{P}(1) \\
& =\left(E_{p_{1}}-I_{a}\left(\alpha_{0} ; \alpha_{0}\right) H_{1}\right) \frac{1}{\sqrt{n}} \partial_{\alpha} U_{n}^{(1)}\left(\alpha_{0}\right)+o_{P}(1) \\
& \stackrel{d}{\rightarrow}\left(E_{p_{1}}-I_{a}\left(\alpha_{0} ; \alpha_{0}\right) H_{1}\right) Y_{1} \quad\left(\text { under } H_{0}^{(1)}\right)
\end{aligned}
$$

By noting that $\partial_{\alpha} U_{n}^{(1)}\left(\hat{\alpha}_{n}\right)=0$, it follows from Taylor's theorem that

$$
\begin{aligned}
-\frac{1}{\sqrt{n}} \partial_{\alpha} U_{n}^{(1)}\left(\tilde{\alpha}_{n}\right) & =\tilde{I}_{a, n}\left(\hat{\alpha}_{n}, \tilde{\alpha}_{n}\right) \sqrt{n}\left(\hat{\alpha}_{n}-\tilde{\alpha}_{n}\right) \\
& =-I_{a}\left(\alpha_{0} ; \alpha_{0}\right) \sqrt{n}\left(\hat{\alpha}_{n}-\tilde{\alpha}_{n}\right)+o_{P}(1) .
\end{aligned}
$$

Since $I_{a}\left(\alpha_{0} ; \alpha_{0}\right)$ is non-singular, by 16 and Slutsky's theorem,

$$
\begin{aligned}
\sqrt{n}\left(\hat{\alpha}_{n}-\tilde{\alpha}_{n}\right) & \stackrel{d}{\rightarrow} I_{a}^{-1}\left(\alpha_{0} ; \alpha_{0}\right)\left(E_{p_{1}}-I_{a}\left(\alpha_{0} ; \alpha_{0}\right) H_{1}\right) Y_{1} \\
& =\left(I_{a}^{-1}\left(\alpha_{0} ; \alpha_{0}\right)-H_{1}\right) Y_{1} \quad\left(\text { under } H_{0}^{(1)}\right) .
\end{aligned}
$$


This completes the proof of (i).

(ii) First, we will show that

$$
H_{2} \frac{1}{\sqrt{n h_{n}}} \partial_{\beta} U_{n}^{(2)}\left(\beta_{0} \mid \alpha_{0}\right)=\sqrt{n h_{n}}\left(\tilde{\beta}_{n}-\beta_{0}\right)+o_{P}(1) \quad\left(\text { under } H_{0}^{(2)}\right) .
$$

From Taylor's theorem with respect to $\beta$,

$$
\frac{1}{\sqrt{n h_{n}}} \partial_{\beta} U_{n}^{(2)}\left(\tilde{\beta}_{n} \mid \hat{\alpha}_{n}\right)=\frac{1}{\sqrt{n h_{n}}} \partial_{\beta} U_{n}^{(2)}\left(\beta_{0} \mid \hat{\alpha}_{n}\right)+\tilde{I}_{b, n}\left(\tilde{\beta}_{n}, \beta_{0}\right) \sqrt{n h_{n}}\left(\tilde{\beta}_{n}-\beta_{0}\right),
$$

where

$$
\tilde{I}_{b, n}\left(\tilde{\beta}_{n}, \beta_{0}\right):=\int_{0}^{1} \frac{1}{n h_{n}} \partial_{\beta}^{2} U_{n}^{(2)}\left(\beta_{0}+u\left(\tilde{\beta}_{n}-\beta_{0}\right) \mid \hat{\alpha}_{n}\right) d u .
$$

Moreover, by Taylor's theorem with respect to $\alpha$,

$$
\frac{1}{\sqrt{n h_{n}}} \partial_{\beta} U_{n}^{(2)}\left(\beta_{0} \mid \hat{\alpha}_{n}\right)=\frac{1}{\sqrt{n h_{n}}} \partial_{\beta} U_{n}^{(2)}\left(\beta_{0} \mid \alpha_{0}\right)+\tilde{I}_{a b, n}\left(\hat{\alpha}_{n}, \alpha_{0}\right) \sqrt{n}\left(\hat{\alpha}_{n}-\alpha_{0}\right),
$$

where

$$
\tilde{I}_{a b, n}\left(\hat{\alpha}_{n}, \alpha_{0}\right):=\int_{0}^{1} \frac{1}{n \sqrt{h_{n}}} \partial_{\alpha} \partial_{\beta} U_{n}^{(2)}\left(\beta_{0} \mid \alpha_{0}+u\left(\hat{\alpha}_{n}-\alpha_{0}\right)\right) d u .
$$

We can show $\tilde{I}_{a b, n}\left(\hat{\alpha}_{n}, \alpha_{0}\right) \stackrel{P}{\rightarrow} 0$ (under $\left.H_{0}^{(2)}\right)$. It follows from $(18)$ and $(19)$ that

$$
\frac{1}{\sqrt{n h_{n}}} \partial_{\beta} U_{n}^{(2)}\left(\tilde{\beta}_{n} \mid \hat{\alpha}_{n}\right)=\frac{1}{\sqrt{n h_{n}}} \partial_{\beta} U_{n}^{(2)}\left(\beta_{0} \mid \alpha_{0}\right)+\tilde{I}_{b, n}\left(\tilde{\beta}_{n}, \beta_{0}\right) \sqrt{n h_{n}}\left(\tilde{\beta}_{n}-\beta_{0}\right)+o_{P}(1) .
$$

Since $H_{2} \partial_{\beta} U_{n}^{(2)}\left(\tilde{\beta}_{n} \mid \hat{\alpha}_{n}\right)=0$, we obtain

$$
H_{2} \frac{1}{\sqrt{n h_{n}}} \partial_{\beta} U_{n}^{(2)}\left(\beta_{0} \mid \alpha_{0}\right)=-H_{2} \tilde{I}_{b, n}\left(\tilde{\beta}_{n}, \beta_{0}\right) \sqrt{n h_{n}}\left(\tilde{\beta}_{n}-\beta_{0}\right)+o_{P}(1) .
$$

On the other hand, we have

$$
\begin{aligned}
-\tilde{I}_{b, n}\left(\tilde{\beta}_{n}, \beta_{0}\right) \stackrel{P}{\rightarrow} I_{b}\left(\theta_{0} ; \theta_{0}\right) & \left(\text { under } H_{0}^{(2)}\right), \\
\frac{1}{\sqrt{n h_{n}}} \partial_{\beta} U_{n}^{(2)}\left(\beta_{0} \mid \alpha_{0}\right) \stackrel{d}{\rightarrow} Y_{2} & \left(\text { under } H_{0}^{(2)}\right), \\
H_{2} \frac{1}{\sqrt{n h_{n}}} \partial_{\beta} U_{n}^{(2)}\left(\beta_{0} \mid \alpha_{0}\right) \stackrel{d}{\rightarrow} H_{2} Y_{2} & \left(\text { under } H_{0}^{(2)}\right) .
\end{aligned}
$$

If we set $\tilde{\beta}_{n}^{\left(p_{2}-r_{2}\right)}=\left(\tilde{\beta}_{n, r_{2}+1}-\beta_{0, r_{2}+1}, \ldots, \tilde{\beta}_{n, p_{2}}-\beta_{0, p_{2}}\right)$, then

$$
\begin{aligned}
H_{2} \tilde{I}_{b, n}\left(\beta_{0}, \beta_{0}\right) \sqrt{n h_{n}}\left(\tilde{\beta}_{n}-\beta_{0}\right) & =\left(\begin{array}{cc}
0 & 0 \\
I_{b, 3}^{-1} \tilde{I}_{b, n, 2}^{T}\left(\beta_{0}, \beta_{0}\right) & I_{b, 3}^{-1} \tilde{I}_{b, n, 3}\left(\beta_{0}, \beta_{0}\right)
\end{array}\right)\left(\begin{array}{c}
0 \\
\sqrt{n h_{n}} \tilde{\beta}_{n}^{\left(p_{2}-r_{2}\right)}
\end{array}\right) \\
& =\left(\begin{array}{cc}
0 \\
I_{b, 3}^{-1} \tilde{I}_{b, n, 3}\left(\beta_{0}, \beta_{0}\right) \sqrt{n h_{n}} \tilde{\beta}_{n}^{\left(p_{2}-r_{2}\right)}
\end{array}\right), \\
H_{2} I_{b}\left(\theta_{0} ; \theta_{0}\right) \sqrt{n h_{n}}\left(\tilde{\beta}_{n}-\beta_{0}\right) & =\left(\begin{array}{cc}
0 & 0 \\
I_{b, 3}^{-1} I_{b, 2}^{T}\left(\theta_{0} ; \theta_{0}\right) & E_{p_{2}-r_{2}}
\end{array}\right)\left(\begin{array}{c}
0 \\
\sqrt{n h_{n}} \tilde{\beta}_{n}^{\left(p_{2}-r_{2}\right)}
\end{array}\right)=\sqrt{n h_{n}}\left(\tilde{\beta}_{n}-\beta_{0}\right) .
\end{aligned}
$$

In an analogous manner to the proof of (15), one has that

$$
\sqrt{n h_{n}}\left(\tilde{\beta}_{n}-\beta_{0}\right)=O_{P}(1) \quad\left(\text { under } H_{0}^{(2)}\right) .
$$


Hence, 21), 222, (27) and (26) implies that

$$
\begin{aligned}
H_{2} \frac{1}{\sqrt{n h_{n}}} \partial_{\beta} U_{n}^{(2)}\left(\beta_{0} \mid \alpha_{0}\right) & =H_{2}\left(I_{b}\left(\theta_{0} ; \theta_{0}\right)+o_{P}(1)\right) \sqrt{n h_{n}}\left(\tilde{\beta}_{n}-\beta_{0}\right)+o_{P}(1) \\
& =H_{2} I_{b}\left(\theta_{0} ; \theta_{0}\right) \sqrt{n h_{n}}\left(\tilde{\beta}_{n}-\beta_{0}\right)+o_{P}(1) \\
& =\sqrt{n h_{n}}\left(\tilde{\beta}_{n}-\beta_{0}\right)+o_{P}(1) .
\end{aligned}
$$

This accords with (17). It follows from (20), 22, 27, 28) and 23) that

$$
\begin{aligned}
\frac{1}{\sqrt{n h_{n}}} \partial_{\beta} U_{n}^{(2)}\left(\tilde{\beta}_{n} \mid \hat{\alpha}_{n}\right) & =\frac{1}{\sqrt{n h_{n}}} \partial_{\beta} U_{n}^{(2)}\left(\beta_{0} \mid \alpha_{0}\right)+\tilde{I}_{b, n}\left(\tilde{\beta}_{n}, \beta_{0}\right) \sqrt{n h_{n}}\left(\tilde{\beta}_{n}-\beta_{0}\right)+o_{P}(1) \\
& =\frac{1}{\sqrt{n h_{n}}} \partial_{\beta} U_{n}^{(2)}\left(\beta_{0} \mid \alpha_{0}\right)-I_{b}\left(\theta_{0} ; \theta_{0}\right) \sqrt{n h_{n}}\left(\tilde{\beta}_{n}-\beta_{0}\right)+o_{P}(1) \\
& =\frac{1}{\sqrt{n h_{n}}} \partial_{\beta} U_{n}^{(2)}\left(\beta_{0} \mid \alpha_{0}\right)-I_{b}\left(\theta_{0} ; \theta_{0}\right) H_{2} \frac{1}{\sqrt{n h_{n}}} \partial_{\beta} U_{n}^{(2)}\left(\beta_{0} \mid \alpha_{0}\right)+o_{P}(1) \\
& =\left(E_{p_{2}}-I_{b}\left(\theta_{0} ; \theta_{0}\right) H_{2}\right) \frac{1}{\sqrt{n h_{n}}} \partial_{\beta} U_{n}^{(2)}\left(\beta_{0} \mid \alpha_{0}\right)+o_{P}(1) \\
& \stackrel{d}{\rightarrow}\left(E_{p_{2}}-I_{b}\left(\theta_{0} ; \theta_{0}\right) H_{2}\right) Y_{2} \quad\left(\text { under } H_{0}^{(2)}\right) .
\end{aligned}
$$

Since we have that under $H_{0}^{(2)}$,

$$
\begin{aligned}
& \sqrt{n h_{n}}\left(\hat{\beta}_{n}-\tilde{\beta}_{n}\right)=O_{P}(1), \\
& \tilde{I}_{b, n}\left(\hat{\beta}_{n}, \tilde{\beta}_{n}\right)=-I_{b}\left(\theta_{0} ; \theta_{0}\right)+o_{P}(1),
\end{aligned}
$$

it follows from Taylor's theorem that under $H_{0}^{(2)}$,

$$
\begin{aligned}
-\frac{1}{\sqrt{n h_{n}}} \partial_{\beta} U_{n}^{(2)}\left(\tilde{\beta}_{n} \mid \hat{\alpha}_{n}\right) & =\tilde{I}_{b, n}\left(\hat{\beta}_{n}, \tilde{\beta}_{n}\right) \sqrt{n h_{n}}\left(\hat{\beta}_{n}-\tilde{\beta}_{n}\right) \\
& =-I_{b}\left(\theta_{0} ; \theta_{0}\right) \sqrt{n h_{n}}\left(\hat{\beta}_{n}-\tilde{\beta}_{n}\right)+o_{P}(1) .
\end{aligned}
$$

Since $I_{b}\left(\theta_{0} ; \theta_{0}\right)$ is non-singular, by 29$)$ and Slutsky's theorem,

$$
\begin{aligned}
\sqrt{n h_{n}}\left(\hat{\beta}_{n}-\tilde{\beta}_{n}\right) \stackrel{d}{\rightarrow} I_{b}^{-1}\left(\theta_{0} ; \theta_{0}\right)\left(E_{p_{2}}-I_{b}\left(\theta_{0}, \theta_{0}\right) H_{2}\right) Y_{2} \\
=\left(I_{b}^{-1}\left(\theta_{0} ; \theta_{0}\right)-H_{2}\right) Y_{2} \quad\left(\text { under } H_{0}^{(2)}\right) .
\end{aligned}
$$

This completes the proof of (ii).

Proof of Theorem 1. (i) By Taylor's theorem, we obtain

$$
U_{n}^{(1)}\left(\tilde{\alpha}_{n}\right)-U_{n}^{(1)}\left(\hat{\alpha}_{n}\right)=\left(\sqrt{n}\left(\hat{\alpha}_{n}-\tilde{\alpha}_{n}\right)\right)^{\top} J_{a, n}\left(\tilde{\alpha}_{n}, \hat{\alpha}_{n}\right) \sqrt{n}\left(\hat{\alpha}_{n}-\tilde{\alpha}_{n}\right),
$$

where

$$
J_{a, n}\left(\tilde{\alpha}_{n}, \hat{\alpha}_{n}\right)=\int_{0}^{1}(1-u) \frac{1}{n} \partial_{\alpha}^{2} U_{n}^{(1)}\left(\hat{\alpha}_{n}+u\left(\tilde{\alpha}_{n}-\hat{\alpha}_{n}\right)\right) d u .
$$

It follows from Lemma 1 that

$$
\sqrt{n}\left(\hat{\alpha}_{n}-\tilde{\alpha}_{n}\right) \stackrel{d}{\rightarrow}\left(I_{a}^{-1}\left(\alpha_{0} ; \alpha_{0}\right)-H_{1}\right) Y_{1} \quad\left(\text { under } H_{0}^{(1)}\right) .
$$

Moreover, we can check that under $H_{0}^{(1)}$,

$$
\begin{gathered}
\hat{\alpha}_{n} \stackrel{P}{\rightarrow} \alpha_{0}, \quad \tilde{\alpha}_{n} \stackrel{P}{\rightarrow} \alpha_{0}, \\
J_{a, n}\left(\tilde{\alpha}_{n}, \hat{\alpha}_{n}\right) \stackrel{P}{\rightarrow}-\frac{1}{2} I_{a}\left(\alpha_{0} ; \alpha_{0}\right) .
\end{gathered}
$$

We set $Y_{1}=I_{a}^{\frac{1}{2}}\left(\alpha_{0} ; \alpha_{0}\right) Z_{1}, Z_{1} \sim N\left(0, E_{p_{1}}\right)$ and

$$
P_{1}=I_{a}^{\frac{1}{2}}\left(\alpha_{0} ; \alpha_{0}\right)\left(I_{a}^{-1}\left(\alpha_{0} ; \alpha_{0}\right)-H_{1}\right) I_{a}^{\frac{1}{2}}\left(\alpha_{0} ; \alpha_{0}\right) .
$$


It follows from the continuous mapping theorem that

$$
\begin{aligned}
\Lambda_{n}^{(1)} & =-2\left(U_{n}^{(1)}\left(\tilde{\alpha}_{n}\right)-U_{n}^{(1)}\left(\hat{\alpha}_{n}\right)\right) \\
& \stackrel{d}{\rightarrow} Y_{1}^{\top}\left(I_{a}^{-1}\left(\alpha_{0} ; \alpha_{0}\right)-H_{1}\right) I_{a}\left(\alpha_{0} ; \alpha_{0}\right)\left(I_{a}^{-1}\left(\alpha_{0} ; \alpha_{0}\right)-H_{1}\right) Y_{1} \\
& =Z_{1}^{\top} I_{a}^{\frac{1}{2}}\left(\alpha_{0} ; \alpha_{0}\right)\left(I_{a}^{-1}\left(\alpha_{0} ; \alpha_{0}\right)-H_{1}\right) I_{a}^{\frac{1}{2}}\left(\alpha_{0} ; \alpha_{0}\right) Z_{1}=Z_{1}^{\top} P_{1} Z_{1} .
\end{aligned}
$$

Since $P_{1}$ is the projection matrix with

$$
\operatorname{rank} P_{1}=\operatorname{trace} P_{1}=r_{1}
$$

we obtain

$$
\Lambda_{n}^{(1)} \stackrel{d}{\rightarrow} \chi_{r_{1}}^{2} \quad\left(\text { under } H_{0}^{(1)}\right),
$$

which implies the first statement of (i).

Next, by Taylor's theorem with respect to $\beta$, one has that under $H_{0}^{(2)}$,

$$
U_{n}^{(2)}\left(\tilde{\beta}_{n} \mid \hat{\alpha}_{n}\right)-U_{n}^{(2)}\left(\hat{\beta}_{n} \mid \hat{\alpha}_{n}\right)=\left(\sqrt{n h_{n}}\left(\hat{\beta}_{n}-\tilde{\beta}_{n}\right)\right)^{\top} J_{b, n}\left(\tilde{\beta}_{n}, \hat{\beta}_{n}\right) \sqrt{n h_{n}}\left(\hat{\beta}_{n}-\tilde{\beta}_{n}\right),
$$

where

$$
J_{b, n}\left(\tilde{\beta}_{n}, \hat{\beta}_{n}\right):=\int_{0}^{1}(1-u) \frac{1}{n h_{n}} \partial_{\beta}^{2} U_{n}^{(2)}\left(\hat{\beta}_{n}+u\left(\tilde{\beta}_{n}-\hat{\beta}_{n}\right) \mid \hat{\alpha}_{n}\right) d u .
$$

It follows from Lemma 1 that

$$
\sqrt{n h_{n}}\left(\hat{\beta}_{n}-\tilde{\beta}_{n}\right) \stackrel{d}{\rightarrow}\left(I_{b}^{-1}\left(\theta_{0} ; \theta_{0}\right)-H_{2}\right) Y_{2} \quad\left(\text { under } H_{0}^{(2)}\right),
$$

and we can check

$$
\begin{gathered}
\hat{\beta}_{n} \stackrel{P}{\rightarrow} \beta_{0}, \quad \tilde{\beta}_{n} \stackrel{P}{\rightarrow} \beta_{0}, \\
J_{b, n}\left(\tilde{\beta}_{n}, \hat{\beta}_{n}\right) \stackrel{P}{\rightarrow}-\frac{1}{2} I_{b}\left(\theta_{0} ; \theta_{0}\right) .
\end{gathered}
$$

Let $Y_{2}=I_{b}^{\frac{1}{2}}\left(\theta_{0} ; \theta_{0}\right) Z_{2}, Z_{2} \sim N\left(0, E_{p_{2}}\right)$ and

$$
P_{2}=I_{b}^{\frac{1}{2}}\left(\theta_{0} ; \theta_{0}\right)\left(I_{b}^{-1}\left(\theta_{0} ; \theta_{0}\right)-H_{2}\right) I_{b}^{\frac{1}{2}}\left(\theta_{0} ; \theta_{0}\right) .
$$

Note that $P_{2}$ is the projection matrix with

$$
\text { rank } P_{2}=\operatorname{trace} P_{2}=r_{2} .
$$

The continuous mapping theorem yields that

$$
\begin{aligned}
\Lambda_{n}^{(2)} & =-2\left(U_{n}^{(2)}\left(\tilde{\beta}_{n} \mid \hat{\alpha}_{n}\right)-U_{n}^{(2)}\left(\hat{\beta}_{n} \mid \hat{\alpha}_{n}\right)\right) \\
& \stackrel{d}{\rightarrow} Y_{2}^{\top}\left(I_{b}^{-1}\left(\theta_{0} ; \theta_{0}\right)-H_{2}\right) I_{b}\left(\theta_{0} ; \theta_{0}\right)\left(I_{b}^{-1}\left(\theta_{0} ; \theta_{0}\right)-H_{2}\right) Y_{2} \\
& =Z_{2}^{\top} I_{b}^{\frac{1}{2}}\left(\theta_{0} ; \theta_{0}\right)\left(I_{b}^{-1}\left(\theta_{0} ; \theta_{0}\right)-H_{2}\right) I_{b}^{\frac{1}{2}}\left(\theta_{0} ; \theta_{0}\right) Z_{2}=Z_{2}^{\top} P_{2} Z_{2} \\
& \sim \chi_{r_{2}}^{2} \quad\left(\text { under } H_{0}^{(2)}\right) .
\end{aligned}
$$

This completes the proof of the Likelihood type.

(ii) By noting that

$$
I_{a, n}\left(\hat{\alpha}_{n}\right) \stackrel{P}{\rightarrow} I_{a}\left(\alpha_{0} ; \alpha_{0}\right) \quad\left(\text { under } H_{0}^{(1)}\right),
$$

it follows from Lemma 1 and the continuous mapping theorem that

$$
\begin{aligned}
W_{n}^{(1)} & =\left(\sqrt{n}\left(\hat{\alpha}_{n}-\tilde{\alpha}_{n}\right)\right)^{\top} I_{a, n}\left(\hat{\alpha}_{n}\right) \sqrt{n}\left(\hat{\alpha}_{n}-\tilde{\alpha}_{n}\right) \\
& \stackrel{d}{\rightarrow} Y_{1}^{\top}\left(I_{a}^{-1}\left(\alpha_{0} ; \alpha_{0}\right)-H_{1}\right) I_{a}\left(\alpha_{0} ; \alpha_{0}\right)\left(I_{a}^{-1}\left(\alpha_{0} ; \alpha_{0}\right)-H_{1}\right) Y_{1} \\
& \sim \chi_{r_{1}}^{2} \quad\left(\text { under } H_{0}^{(1)}\right) .
\end{aligned}
$$


Moreover, we check

$$
I_{b, n}\left(\hat{\beta}_{n} \mid \hat{\alpha}_{n}\right) \stackrel{P}{\rightarrow} I_{b}\left(\theta_{0} ; \theta_{0}\right) \quad\left(\text { under } H_{0}^{(2)}\right),
$$

and it holds

$$
\begin{aligned}
W_{n}^{(2)} & =\left(\sqrt{n h_{n}}\left(\hat{\beta}_{n}-\tilde{\beta}_{n}\right)\right)^{\top} I_{b, n}\left(\hat{\beta}_{n} \mid \hat{\alpha}_{n}\right) \sqrt{n h_{n}}\left(\hat{\beta}_{n}-\tilde{\beta}_{n}\right) \\
& \stackrel{d}{\rightarrow} Y_{2}^{\top}\left(I_{b}^{-1}\left(\theta_{0} ; \theta_{0}\right)-H_{2}\right) I_{b}\left(\theta_{0} ; \theta_{0}\right)\left(I_{b}^{-1}\left(\theta_{0} ; \theta_{0}\right)-H_{2}\right) Y_{2} \\
& \sim \chi_{r_{2}}^{2} \quad\left(\text { under } H_{0}^{(2)}\right) .
\end{aligned}
$$

This completes the proof of the Wald type.

(iii) From the proof of Lemma 1, it holds

$$
\frac{1}{\sqrt{n}} \partial_{\alpha} U_{n}^{(1)}\left(\tilde{\alpha}_{n}\right) \stackrel{d}{\rightarrow}\left(E_{p_{1}}-I_{a}\left(\alpha_{0} ; \alpha_{0}\right) H_{1}\right) Y_{1} \quad\left(\text { under } H_{0}^{(1)}\right) .
$$

It follows that

$$
\left.\bar{I}_{a, n}\left(\hat{\alpha}_{n}\right) \stackrel{P}{\rightarrow} I_{a}^{-1}\left(\alpha_{0} ; \alpha_{0}\right) \quad \text { (under } H_{0}^{(1)}\right) .
$$

Hence we obtain from the continuous mapping theorem that

$$
\begin{aligned}
R_{n}^{(1)} & =\left(\frac{1}{\sqrt{n}} \partial_{\alpha} U_{n}^{(1)}\left(\tilde{\alpha}_{n}\right)\right)^{\top} \bar{I}_{a, n}\left(\hat{\alpha}_{n}\right) \frac{1}{\sqrt{n}} \partial_{\alpha} U_{n}^{(1)}\left(\tilde{\alpha}_{n}\right) \\
& \stackrel{d}{\rightarrow} Y_{1}^{\top}\left(E_{p_{1}}-I_{a}\left(\alpha_{0} ; \alpha_{0}\right) H_{1}\right)^{\top} I_{a}^{-1}\left(\alpha_{0} ; \alpha_{0}\right)\left(E_{p_{1}}-I_{a}\left(\alpha_{0} ; \alpha_{0}\right) H_{1}\right) Y_{1} \\
& =Y_{1}^{\top}\left(E_{p_{1}}-I_{a}\left(\alpha_{0} ; \alpha_{0}\right) H_{1}\right)^{\top} I_{a}^{-1}\left(\alpha_{0} ; \alpha_{0}\right) I_{a}\left(\alpha_{0} ; \alpha_{0}\right) I_{a}^{-1}\left(\alpha_{0} ; \alpha_{0}\right)\left(E_{p_{1}}-I_{a}\left(\alpha_{0} ; \alpha_{0}\right) H_{1}\right) Y_{1} \\
& =Y_{1}^{\top}\left(I_{a}^{-1}\left(\alpha_{0} ; \alpha_{0}\right)-H_{1}\right) I_{a}\left(\alpha_{0} ; \alpha_{0}\right)\left(I_{a}^{-1}\left(\alpha_{0} ; \alpha_{0}\right)-H_{1}\right) Y_{1} \\
& \sim \chi_{r_{1}}^{2} \quad\left(\text { under } H_{0}^{(1)}\right) .
\end{aligned}
$$

Furthermore, it follows from the proof of Lemma 1 that

$$
\frac{1}{\sqrt{n h_{n}}} \partial_{\beta} U_{n}^{(2)}\left(\tilde{\beta}_{n} \mid \hat{\alpha}_{n}\right) \stackrel{d}{\rightarrow}\left(E_{p_{2}}-I_{b}\left(\theta_{0}, \theta_{0}\right) H_{2}\right) Y_{2} \quad\left(\text { under } H_{0}^{(2)}\right),
$$

and we can check

$$
\bar{I}_{b, n}\left(\hat{\beta}_{n} \mid \hat{\alpha}_{n}\right) \stackrel{P}{\rightarrow} I_{b}^{-1}\left(\theta_{0} ; \theta_{0}\right) \quad\left(\text { under } H_{0}^{(2)}\right) .
$$

Thus, one has

$$
\begin{aligned}
R_{n}^{(2)} & =\left(\frac{1}{\sqrt{n h_{n}}} \partial_{\beta} U_{n}^{(2)}\left(\tilde{\beta}_{n} \mid \hat{\alpha}_{n}\right)\right)^{\top} \bar{I}_{b, n}\left(\hat{\beta}_{n} \mid \hat{\alpha}_{n}\right) \frac{1}{\sqrt{n h_{n}}} \partial_{\beta} U_{n}^{(2)}\left(\tilde{\beta}_{n} \mid \hat{\alpha}_{n}\right) \\
& \stackrel{d}{\rightarrow} Y_{2}^{\top}\left(E_{p_{2}}-I_{b}\left(\theta_{0} ; \theta_{0}\right) H_{2}\right)^{\top} I_{b}^{-1}\left(\theta_{0} ; \theta_{0}\right)\left(E_{p_{2}}-I_{b}\left(\theta_{0} ; \theta_{0}\right) H_{2}\right) Y_{2} \\
& =Y_{2}^{\top}\left(E_{p_{2}}-I_{b}\left(\theta_{0} ; \theta_{0}\right) H_{2}\right)^{\top} I_{b}^{-1}\left(\theta_{0} ; \theta_{0}\right) I_{b}\left(\theta_{0} ; \theta_{0}\right) I_{b}^{-1}\left(\theta_{0} ; \theta_{0}\right)\left(E_{p_{2}}-I_{b}\left(\theta_{0} ; \theta_{0}\right) H_{2}\right) Y_{2} \\
& =Y_{2}^{\top}\left(I_{b}^{-1}\left(\theta_{0} ; \theta_{0}\right)-H_{2}\right) I_{b}\left(\theta_{0} ; \theta_{0}\right)\left(I_{b}^{-1}\left(\theta_{0} ; \theta_{0}\right)-H_{2}\right) Y_{2} \\
& \sim \chi_{r_{2}}^{2} \quad\left(\text { under } H_{0}^{(2)}\right) .
\end{aligned}
$$

This completes the proof of the Rao type.

Next, we will prove the following lemma to show Theorem 2.

Lemma 2 Assume A1-A7 and B1. If $h_{n} \rightarrow 0$ and $n h_{n} \rightarrow \infty$, then

(i) $\tilde{\alpha}_{n} \stackrel{P}{\rightarrow} \alpha^{*} \quad$ (under $\left.H_{1}^{(1)}\right)$,

(ii) $\tilde{\beta}_{n} \stackrel{P}{\rightarrow} \beta^{*} \quad$ (under $H_{1}^{(2)}$ ). 
Proof. (i) From the definition of $\bar{U}_{1}\left(\alpha ; \alpha_{1}^{*}\right)$, it holds

$$
\sup _{\alpha \in \Theta_{\alpha}}\left|\frac{1}{n} U_{n}^{(1)}(\alpha)-\bar{U}_{1}\left(\alpha ; \alpha_{1}^{*}\right)\right| \stackrel{P}{\rightarrow} 0 \quad\left(\text { under } H_{1}^{(1)}\right) .
$$

B1-(a) implies the following : for all $\varepsilon>0$, there exists $\delta>0$ such that for all $\alpha \in \tilde{\Theta}_{\alpha}$,

$$
\left|\alpha-\alpha^{*}\right| \geq \varepsilon \Longrightarrow \bar{U}_{1}\left(\alpha^{*} ; \alpha_{1}^{*}\right)-\bar{U}_{1}\left(\alpha ; \alpha_{1}^{*}\right)>\delta .
$$

Therefore we obtain

$$
\begin{aligned}
0 & \leq P\left(\left|\tilde{\alpha}_{n}-\alpha^{*}\right| \geq \varepsilon\right) \\
& \leq P\left(\bar{U}_{1}\left(\alpha^{*} ; \alpha_{1}^{*}\right)-\bar{U}_{1}\left(\tilde{\alpha}_{n} ; \alpha_{1}^{*}\right)>\delta\right) \\
& =P\left(\bar{U}_{1}\left(\alpha^{*} ; \alpha_{1}^{*}\right)-\frac{1}{n} U_{n}^{(1)}\left(\alpha^{*}\right)+\frac{1}{n} U_{n}^{(1)}\left(\alpha^{*}\right)-\frac{1}{n} U_{n}^{(1)}\left(\tilde{\alpha}_{n}\right)+\frac{1}{n} U_{n}^{(1)}\left(\tilde{\alpha}_{n}\right)-\bar{U}_{1}\left(\tilde{\alpha}_{n} ; \alpha_{1}^{*}\right)>\delta\right) \\
& \leq 2 P\left(\sup _{\alpha \in \Theta_{\alpha}}\left|\frac{1}{n} U_{n}^{(1)}(\alpha)-\bar{U}_{1}\left(\alpha ; \alpha_{1}^{*}\right)\right|>\frac{\delta}{3}\right)+P\left(\frac{1}{n} U_{n}^{(1)}\left(\alpha^{*}\right)-\frac{1}{n} U_{n}^{(1)}\left(\tilde{\alpha}_{n}\right)>\frac{\delta}{3}\right) \\
& \rightarrow 0(n \rightarrow \infty),
\end{aligned}
$$

which implies the statement of (i).

(ii) For sake of simplicity, $\alpha_{1}^{*}$ denotes the true value of $\alpha$ under $H_{1}^{(2)}$. From the definition of $\bar{U}_{2}\left(\alpha, \beta ; \beta_{1}^{*}\right)$, it holds

$$
\sup _{\theta \in \Theta}\left|L_{n}(\alpha, \beta)-\bar{U}_{2}\left(\alpha, \beta ; \beta_{1}^{*}\right)\right| \stackrel{P}{\rightarrow} 0 \quad\left(\text { under } H_{1}^{(2)}\right),
$$

where

$$
L_{n}(\alpha, \beta)=\frac{1}{n h_{n}} U_{n}^{(2)}(\beta \mid \alpha)-\frac{1}{n h_{n}} U_{n}^{(2)}\left(\beta_{1}^{*} \mid \alpha\right)
$$

B1-(b) implies the following : for all $\varepsilon>0$, there exists $\delta>0$ such that for all $\beta \in \tilde{\Theta}_{\beta}$,

$$
\left|\beta-\beta^{*}\right| \geq \varepsilon \Longrightarrow \bar{U}_{2}\left(\alpha_{1}^{*}, \beta^{*} ; \beta_{1}^{*}\right)-\bar{U}_{2}\left(\alpha_{1}^{*}, \beta ; \beta_{1}^{*}\right)>\delta \text {. }
$$

Hence it holds

$$
\begin{aligned}
0 & \leq P\left(\left|\tilde{\beta}_{n}-\beta^{*}\right| \geq \varepsilon\right) \\
& \leq P\left(\bar{U}_{2}\left(\alpha_{1}^{*}, \beta^{*} ; \beta_{1}^{*}\right)-\bar{U}_{2}\left(\alpha_{1}^{*}, \tilde{\beta}_{n} ; \beta_{1}^{*}\right)>\delta\right) \\
& =P\left(\bar{U}_{2}\left(\alpha_{1}^{*}, \beta^{*} ; \beta_{1}^{*}\right)-L_{n}\left(\hat{\alpha}_{n}, \beta^{*}\right)+L_{n}\left(\hat{\alpha}_{n}, \beta^{*}\right)-L_{n}\left(\hat{\alpha}_{n}, \tilde{\beta}_{n}\right)+L_{n}\left(\hat{\alpha}_{n}, \tilde{\beta}_{n}\right)-\bar{U}_{2}\left(\alpha_{1}^{*}, \tilde{\beta}_{n} ; \beta_{1}^{*}\right)>\delta\right) \\
& \leq 2 P\left(\sup _{\beta \in \Theta_{\beta}}\left|L_{n}\left(\hat{\alpha}_{n}, \beta\right)-\bar{U}_{2}\left(\alpha_{1}^{*}, \beta ; \beta_{1}^{*}\right)\right|>\frac{\delta}{3}\right)+P\left(L_{n}\left(\hat{\alpha}_{n}, \beta^{*}\right)-L_{n}\left(\hat{\alpha}_{n}, \tilde{\beta}_{n}\right)>\frac{\delta}{3}\right) \\
& =2 P\left(\sup _{\beta \in \Theta_{\beta}}\left|L_{n}\left(\hat{\alpha}_{n}, \beta\right)-\bar{U}_{2}\left(\alpha_{1}^{*}, \beta ; \beta_{1}^{*}\right)\right|>\frac{\delta}{3}\right),
\end{aligned}
$$

where

$$
\begin{aligned}
P\left(\sup _{\beta \in \Theta_{\beta}}\left|L_{n}\left(\hat{\alpha}_{n}, \beta\right)-\bar{U}_{2}\left(\alpha_{1}^{*}, \beta ; \beta_{1}^{*}\right)\right|>\frac{\delta}{3}\right) \leq & P\left(\sup _{\beta \in \Theta_{\beta}}\left|L_{n}\left(\hat{\alpha}_{n}, \beta\right)-L_{n}\left(\alpha_{1}^{*}, \beta\right)\right|>\frac{\delta}{6}\right) \\
& +P\left(\sup _{\beta \in \Theta_{\beta}}\left|L_{n}\left(\alpha_{1}^{*}, \beta\right)-\bar{U}_{2}\left(\alpha_{1}^{*}, \beta ; \beta_{1}^{*}\right)\right|>\frac{\delta}{6}\right) \\
\leq & P\left(\sup _{\beta \in \Theta_{\beta}}\left|L_{n}\left(\hat{\alpha}_{n}, \beta\right)-L_{n}\left(\alpha_{1}^{*}, \beta\right)\right|>\frac{\delta}{6}\right) \\
& +P\left(\sup _{\theta \in \Theta}\left|L_{n}(\alpha, \beta)-\bar{U}_{2}\left(\alpha, \beta ; \beta_{1}^{*}\right)\right|>\frac{\delta}{6}\right) .
\end{aligned}
$$


The second term on the right hand side of the inequality (30) converges to 0 under $H_{0}^{(2)}$. It follows from Taylor's theorem that

$$
\begin{aligned}
\left|L_{n}\left(\hat{\alpha}_{n}, \beta\right)-L_{n}\left(\alpha_{1}^{*}, \beta\right)\right| & =\left|\int_{0}^{1} \partial_{\alpha} L_{n}\left(\alpha_{1}^{*}+u\left(\hat{\alpha}_{n}-\alpha_{1}^{*}\right), \beta\right) d u\left(\hat{\alpha}_{n}-\alpha_{1}^{*}\right)\right| \\
& \leq \int_{0}^{1}\left|\partial_{\alpha} L_{n}\left(\alpha_{1}^{*}+u\left(\hat{\alpha}_{n}-\alpha_{1}^{*}\right), \beta\right)\right| d u\left|\hat{\alpha}_{n}-\alpha_{1}^{*}\right| \\
& \leq \sup _{\theta \in \Theta}\left|\partial_{\alpha} L_{n}(\alpha, \beta)\right|\left|\hat{\alpha}_{n}-\alpha_{1}^{*}\right|,
\end{aligned}
$$

and it is easy to check

$$
\left|\hat{\alpha}_{n}-\alpha_{1}^{*}\right|=o_{P}(1), \quad \sup _{\theta \in \Theta}\left|\partial_{\alpha} L_{n}(\alpha, \beta)\right|=O_{P}(1) .
$$

Therefore, the first term on the right hand side of the inequality 30 converges to 0 , which implies $\tilde{\beta}_{n} \stackrel{P}{\rightarrow}$ $\beta^{*}$.

Proof of Theorem 2. (i) Since Lemma 2 implies that under $H_{1}^{(1)}$,

$$
\begin{gathered}
\hat{\alpha}_{n} \stackrel{P}{\rightarrow} \alpha_{1}^{*}, \quad \tilde{\alpha}_{n} \stackrel{P}{\rightarrow} \alpha^{*} \neq \alpha_{1}^{*}, \\
\sup _{\alpha \in \Theta_{\alpha}}\left|\frac{1}{n} U_{n}^{(1)}(\alpha)-\bar{U}_{1}\left(\alpha ; \alpha_{1}^{*}\right)\right| \stackrel{P}{\rightarrow} 0,
\end{gathered}
$$

we obtain

$$
\begin{aligned}
\frac{1}{n} \Lambda_{n}^{(1)} & =\frac{2}{n}\left(U_{n}^{(1)}\left(\hat{\alpha}_{n}\right)-U_{n}^{(1)}\left(\tilde{\alpha}_{n}\right)\right) \\
& \stackrel{P}{\rightarrow} 2\left(\bar{U}_{1}\left(\alpha_{1}^{*} ; \alpha_{1}^{*}\right)-\bar{U}_{1}\left(\alpha^{*} ; \alpha_{1}^{*}\right)\right)>0 .
\end{aligned}
$$

Therefore, for all $\varepsilon \in(0,1)$,

$$
0 \leq P\left(\Lambda_{n}^{(1)} \leq \chi_{r_{1}, \varepsilon}^{2}\right)=P\left(\frac{1}{n} \Lambda_{n}^{(1)} \leq \frac{\chi_{r_{1}, \varepsilon}^{2}}{n}\right) \rightarrow 0 \quad\left(\text { under } H_{1}^{(1)}, n \rightarrow \infty\right),
$$

which implies the first statement of (i).

Next, we consider $\Lambda_{n}^{(2)}$ under $H_{1}^{(2)}$. It holds

$$
\begin{aligned}
\frac{1}{n h_{n}} \Lambda_{n}^{(2)} & =\frac{2}{n h_{n}}\left(U_{n}^{(2)}\left(\hat{\beta}_{n} \mid \hat{\alpha}_{n}\right)-U_{n}^{(2)}\left(\tilde{\beta}_{n} \mid \hat{\alpha}_{n}\right)\right) \\
& =\frac{2}{n h_{n}}\left(U_{n}^{(2)}\left(\hat{\beta}_{n} \mid \hat{\alpha}_{n}\right)-U_{n}^{(2)}\left(\beta_{1}^{*} \mid \hat{\alpha}_{n}\right)\right)+\frac{2}{n h_{n}}\left(U_{n}^{(2)}\left(\beta_{1}^{*} \mid \hat{\alpha}_{n}\right)-U_{n}^{(2)}\left(\tilde{\beta}_{n} \mid \hat{\alpha}_{n}\right)\right) \\
& \geq \frac{2}{n h_{n}}\left(U_{n}^{(2)}\left(\beta_{1}^{*} \mid \hat{\alpha}_{n}\right)-U_{n}^{(2)}\left(\tilde{\beta}_{n} \mid \hat{\alpha}_{n}\right)\right) .
\end{aligned}
$$

Lemma 2 implies that under $H_{1}^{(2)}$,

$$
\begin{gathered}
\hat{\alpha}_{n} \stackrel{P}{\rightarrow} \alpha_{1}^{*}, \quad \tilde{\beta}_{n} \stackrel{P}{\rightarrow} \beta^{*} \neq \beta_{1}^{*}, \\
\sup _{\theta \in \Theta}\left|L_{n}(\alpha, \beta)-\bar{U}_{2}\left(\alpha, \beta ; \beta_{1}^{*}\right)\right| \stackrel{P}{\rightarrow} 0 .
\end{gathered}
$$

Noting that $\bar{U}_{2}\left(\alpha_{1}^{*}, \beta_{1}^{*} ; \beta_{1}^{*}\right)=0$ and $L_{n}(\alpha, \beta)=\frac{1}{n h_{n}}\left(U_{n}^{(2)}(\beta \mid \alpha)-U_{n}^{(2)}\left(\beta_{1}^{*} \mid \alpha\right)\right)$, one has

$$
\frac{2}{n h_{n}}\left(U_{n}^{(2)}\left(\beta_{1}^{*} \mid \hat{\alpha}_{n}\right)-U_{n}^{(2)}\left(\tilde{\beta}_{n} \mid \hat{\alpha}_{n}\right)\right) \stackrel{P}{\rightarrow}-2 \bar{U}_{2}\left(\alpha_{1}^{*}, \beta^{*} ; \beta_{1}^{*}\right)>0 .
$$


Therefore, for all $\varepsilon \in(0,1)$,

$$
\begin{aligned}
0 \leq P\left(\Lambda_{n}^{(2)} \leq \chi_{r_{2}, \varepsilon}^{2}\right) & =P\left(\frac{1}{n h_{n}} \Lambda_{n}^{(2)} \leq \frac{\chi_{r_{2}, \varepsilon}^{2}}{n h_{n}}\right) \\
& \leq P\left(\frac{2}{n h_{n}}\left(U_{n}^{(2)}\left(\beta_{1}^{*} \mid \hat{\alpha}_{n}\right)-U_{n}^{(2)}\left(\tilde{\beta}_{n} \mid \hat{\alpha}_{n}\right)\right) \leq \frac{\chi_{r_{2}, \varepsilon}^{2}}{n h_{n}}\right) \\
& \rightarrow 0 \quad\left(\text { under } H_{1}^{(2)}, n \rightarrow \infty\right) .
\end{aligned}
$$

This completes the proof of the Likelihood type.

(ii) We have that under $H_{1}^{(1)}$,

$$
\begin{aligned}
& \hat{\alpha}_{n}-\tilde{\alpha}_{n} \stackrel{P}{\rightarrow} \alpha_{1}^{*}-\alpha^{*} \neq 0, \\
& I_{a, n}\left(\hat{\alpha}_{n}\right) \stackrel{P}{\rightarrow} I_{a}\left(\alpha_{1}^{*} ; \alpha_{1}^{*}\right) .
\end{aligned}
$$

Hence

$$
\begin{aligned}
\frac{1}{n} W_{n}^{(1)} & =\left(\hat{\alpha}_{n}-\tilde{\alpha}_{n}\right)^{\top} I_{a, n}\left(\hat{\alpha}_{n}\right)\left(\hat{\alpha}_{n}-\tilde{\alpha}_{n}\right) \\
& \stackrel{P}{\rightarrow}\left(\alpha_{1}^{*}-\alpha^{*}\right)^{\top} I_{a}\left(\alpha_{1}^{*} ; \alpha_{1}^{*}\right)\left(\alpha_{1}^{*}-\alpha^{*}\right)>0
\end{aligned}
$$

because of $\mathbf{A} 7$. Therefore, for all $\varepsilon \in(0,1)$,

$$
P\left(W_{n}^{(1)} \geq \chi_{r_{1}, \varepsilon}^{2}\right)=P\left(\frac{1}{n} W_{n}^{(1)} \geq \frac{\chi_{r_{1}, \varepsilon}^{2}}{n}\right) \rightarrow 1 \quad\left(\text { under } H_{1}^{(1)}\right) .
$$

Moreover, Lemma 2 implies that under $H_{1}^{(2)}$,

$$
\begin{aligned}
& \hat{\beta}_{n}-\tilde{\beta}_{n} \stackrel{P}{\rightarrow} \beta_{1}^{*}-\beta^{*} \neq 0 \\
& I_{b, n}\left(\hat{\beta}_{n} \mid \hat{\alpha}_{n}\right) \stackrel{P}{\rightarrow} I_{b}\left(\theta_{1}^{*} ; \theta_{1}^{*}\right) .
\end{aligned}
$$

Hence

$$
\begin{aligned}
\frac{1}{n h_{n}} W_{n}^{(2)} & =\left(\hat{\beta}_{n}-\tilde{\beta}_{n}\right)^{\top} I_{b, n}\left(\hat{\beta}_{n} \mid \hat{\alpha}_{n}\right)\left(\hat{\beta}_{n}-\tilde{\beta}_{n}\right) \\
& \stackrel{P}{\rightarrow}\left(\beta_{1}^{*}-\beta^{*}\right)^{\top} I_{b}\left(\theta_{1}^{*} ; \theta_{1}^{*}\right)\left(\beta_{1}^{*}-\beta^{*}\right)>0 .
\end{aligned}
$$

Therefore, for all $\varepsilon \in(0,1)$,

$$
P\left(W_{n}^{(2)} \geq \chi_{r_{2}, \varepsilon}^{2}\right)=P\left(\frac{1}{n h_{n}} W_{n}^{(2)} \geq \frac{\chi_{r_{2}, \varepsilon}^{2}}{n h_{n}}\right) \rightarrow 1 \quad\left(\text { under } H_{1}^{(2)}\right) .
$$

This completes the proof of the Wald type.

(iii) From Taylor's theorem, it holds

$$
\frac{1}{n} \partial_{\alpha} U_{n}^{(1)}\left(\tilde{\alpha}_{n}\right)=-\int_{0}^{1} \frac{1}{n} \partial_{\alpha}^{2} U_{n}^{(1)}\left(\hat{\alpha}_{n}+u\left(\tilde{\alpha}_{n}-\hat{\alpha}_{n}\right)\right) d u\left(\hat{\alpha}_{n}-\tilde{\alpha}_{n}\right) .
$$

It is shown that under $H_{1}^{(1)}$,

$$
\begin{gathered}
\hat{\alpha}_{n}-\tilde{\alpha}_{n} \stackrel{P}{\rightarrow} \alpha_{1}^{*}-\alpha^{*} \neq 0, \\
-\int_{0}^{1} \frac{1}{n} \partial_{\alpha}^{2} U_{n}^{(1)}\left(\hat{\alpha}_{n}+u\left(\tilde{\alpha}_{n}-\hat{\alpha}_{n}\right)\right) d u \stackrel{P}{\rightarrow} \int_{0}^{1} I_{a}\left(\alpha_{1}^{*}+u\left(\alpha^{*}-\alpha_{1}^{*}\right) ; \alpha_{1}^{*}\right) d u .
\end{gathered}
$$

Hence it follows from B2-(a) that

$$
\frac{1}{n} \partial_{\alpha} U_{n}^{(1)}\left(\tilde{\alpha}_{n}\right) \stackrel{P}{\rightarrow} \int_{0}^{1} I_{a}\left(\alpha_{1}^{*}+u\left(\alpha^{*}-\alpha_{1}^{*}\right) ; \alpha_{1}^{*}\right) d u\left(\alpha_{1}^{*}-\alpha^{*}\right)=: c_{1} \neq 0,
$$


and

$$
\begin{aligned}
\frac{1}{n} R_{n}^{(1)} & =\left(\frac{1}{n} \partial_{\alpha} U_{n}^{(1)}\left(\tilde{\alpha}_{n}\right)\right)^{\top} \bar{I}_{a, n}\left(\hat{\alpha}_{n}\right) \frac{1}{n} \partial_{\alpha} U_{n}^{(1)}\left(\tilde{\alpha}_{n}\right) \\
& \stackrel{P}{\rightarrow} c_{1}^{\top} I_{a}^{-1}\left(\alpha_{1}^{*}, \alpha_{1}^{*}\right) c_{1}>0 .
\end{aligned}
$$

Therefore, for all $\varepsilon \in(0,1)$,

$$
P\left(R_{n}^{(1)} \geq \chi_{r_{1}, \varepsilon}^{2}\right)=P\left(\frac{1}{n} R_{n}^{(1)} \geq \frac{\chi_{r_{1}, \varepsilon}^{2}}{n}\right) \rightarrow 1 \quad\left(\text { under } H_{1}^{(1)}\right) .
$$

Furthermore, it follows from Taylor's theorem with respect to $\beta$ that under $H_{1}^{(2)}$,

$$
\frac{1}{n h_{n}} \partial_{\beta} U_{n}^{(2)}\left(\tilde{\beta}_{n} \mid \hat{\alpha}_{n}\right)=-\int_{0}^{1} \frac{1}{n h_{n}} \partial_{\beta}^{2} U_{n}^{(2)}\left(\hat{\beta}_{n}+u\left(\tilde{\beta}_{n}-\hat{\beta}_{n}\right) \mid \hat{\alpha}_{n}\right) d u\left(\hat{\beta}_{n}-\tilde{\beta}_{n}\right),
$$

and we have

$$
\begin{gathered}
\hat{\beta}_{n}-\tilde{\beta}_{n} \stackrel{P}{\rightarrow} \beta_{1}^{*}-\beta^{*} \neq 0, \\
\left.-\int_{0}^{1} \frac{1}{n h_{n}} \partial_{\beta}^{2} U_{n}^{(2)}\left(\hat{\beta}_{n}+u\left(\tilde{\beta}_{n}-\hat{\beta}_{n}\right) \mid \hat{\alpha}_{n}\right)\right) d u \stackrel{P}{\rightarrow} \int_{0}^{1} I_{b}\left(\left(\alpha_{1}^{*}, \beta_{1}^{*}+u\left(\beta^{*}-\beta_{1}^{*}\right)\right) ; \theta_{1}^{*}\right) d u .
\end{gathered}
$$

Hence it follows from B2-(b) that

$$
\frac{1}{n h_{n}} \partial_{\beta} U_{n}^{(2)}\left(\tilde{\beta}_{n} \mid \hat{\alpha}_{n}\right) \stackrel{P}{\rightarrow} \int_{0}^{1} I_{b}\left(\left(\alpha_{1}^{*}, \beta_{1}^{*}+u\left(\beta^{*}-\beta_{1}^{*}\right)\right) ; \theta_{1}^{*}\right) d u\left(\beta_{1}^{*}-\beta^{*}\right)=: c_{2} \neq 0,
$$

and

$$
\begin{aligned}
\frac{1}{n h_{n}} R_{n}^{(2)} & =\left(\frac{1}{n h_{n}} \partial_{\beta} U_{n}^{(2)}\left(\tilde{\beta}_{n} \mid \hat{\alpha}_{n}\right)\right)^{\top} \bar{I}_{b, n}\left(\hat{\beta}_{n} \mid \hat{\alpha}_{n}\right) \frac{1}{n h_{n}} \partial_{\beta} U_{n}^{(2)}\left(\tilde{\beta}_{n} \mid \hat{\alpha}_{n}\right) \\
& \stackrel{P}{\rightarrow} c_{2}^{\top} I_{b}^{-1}\left(\theta_{1}^{*}, \theta_{1}^{*}\right) c_{2}>0 .
\end{aligned}
$$

Therefore, for all $\varepsilon \in(0,1)$,

$$
P\left(R_{n}^{(2)} \geq \chi_{r_{2}, \varepsilon}^{2}\right)=P\left(\frac{1}{n h_{n}} R_{n}^{(2)} \geq \frac{\chi_{r_{2}, \varepsilon}^{2}}{n h_{n}}\right) \rightarrow 1 \quad\left(\text { under } H_{1}^{(2)}\right) .
$$

This completes the proof of the Rao type.

Finally, we will prove Theorem 3 . In order to proof this theorem, we use the following lemma.

Lemma 3 Assume A1-A7 and C1. If $h_{n} \rightarrow 0, n h_{n} \rightarrow \infty$ and $n h_{n}^{2} \rightarrow 0$, then

(i) $\sqrt{n}\left(\hat{\alpha}_{n}-\alpha_{0}\right) \stackrel{d}{\rightarrow} Y_{1}^{\prime} \sim N\left(u_{\alpha}, I_{a}^{-1}\left(\alpha_{0} ; \alpha_{0}\right)\right.$ ) (under $\left.H_{1, n}^{(1)}\right)$,

(ii) $\sqrt{n h_{n}}\left(\hat{\beta}_{n}-\beta_{0}\right) \stackrel{d}{\rightarrow} Y_{2}^{\prime} \sim N\left(u_{\beta}, I_{b}^{-1}\left(\theta_{0} ; \theta_{0}\right)\right) \quad$ (under $\left.H_{1, n}^{(2)}\right)$.

Proof. (i) First of all, it is shown that

$$
\frac{1}{\sqrt{n}} \partial_{\alpha} U_{n}^{(1)}\left(\alpha_{0}\right) \stackrel{d}{\rightarrow} L_{1} \sim N\left(I_{a}\left(\alpha_{0} ; \alpha_{0}\right) u_{\alpha}, I_{a}\left(\alpha_{0} ; \alpha_{0}\right)\right) \quad\left(\text { under } H_{1, n}^{(1)}\right) .
$$

For sake of simplicity, we use the following symbols:

$$
\begin{aligned}
& X_{t_{i}^{n}}=\left(X_{i, 1}, \ldots, X_{i, d}\right)^{\top}, S\left(X_{t_{i-1}^{n}}, \alpha\right)=S_{i-1}(\alpha)=\left(S_{i-1, p q}(\alpha)\right)_{1 \leq p, q \leq d}, u_{\alpha}=\left(u_{\alpha, 1}, \ldots, u_{\alpha, p_{1}}\right)^{\top}, \\
& R_{i-1}\left(h_{n}\right):=R\left(\theta, h_{n}, X_{t_{i-1}^{n}}\right), \mathcal{G}_{i}^{n}:=\sigma\left(W_{s}, s \leq t_{i}^{n}\right) .
\end{aligned}
$$


It follows that

$$
\begin{aligned}
U_{n}^{(1)}(\alpha) & =-\frac{1}{2} \sum_{i=1}^{n}\left\{h_{n}^{-1} S^{-1}\left(X_{t_{i-1}^{n}}, \alpha\right)\left[\left(X_{t_{i}^{n}}-X_{t_{i-1}^{n}}\right)^{\otimes 2}\right]+\log \operatorname{det} S\left(X_{t_{i-1}^{n}}, \alpha\right)\right\} \\
& =-\frac{1}{2 h_{n}} \sum_{i=1}^{n} \sum_{p, q}\left\{S_{i-1, p q}^{-1}(\alpha)\left(X_{i, p}-X_{i-1, p}\right)\left(X_{i, q}-X_{i-1, q}\right)\right\}-\frac{1}{2} \sum_{i=1}^{n} \log \operatorname{det} S_{i-1}(\alpha) .
\end{aligned}
$$

For $l_{1}=1, \ldots, p_{1}$, we have

$$
\left(\partial_{\alpha_{l_{1}}} S^{-1}\right)_{i-1}(\alpha)=-\left(S^{-1}\left(\partial_{\alpha_{l_{1}}} S\right) S^{-1}\right)_{i-1}(\alpha),\left(\partial_{\alpha_{l_{1}}} \log \operatorname{det} S\right)_{i-1}(\alpha)=\operatorname{tr}\left(\left(S^{-1} \partial_{\alpha_{l_{1}}} S\right)_{i-1}(\alpha)\right)
$$

and we set

$$
\begin{aligned}
& \partial_{\alpha_{l_{1}}} U_{n}^{(1)}\left(\alpha_{0}\right):=\sum_{i=1}^{n}\left(\xi_{i, 1}^{l_{1}}+\xi_{i, 2}^{l_{1}}\right), \\
& \xi_{i, 1}^{l_{1}}:=\frac{1}{2 h_{n}} \sum_{p, q}\left(S^{-1}\left(\partial_{\alpha_{l_{1}}} S\right) S^{-1}\right)_{i-1, p q}\left(\alpha_{0}\right)\left(X_{i, p}-X_{i-1, p}\right)\left(X_{i, q}-X_{i-1, q}\right), \\
& \xi_{i, 2}^{l_{1}}:=-\frac{1}{2} \operatorname{tr}\left(\left(S^{-1} \partial_{\alpha_{l_{1}}} S\right)_{i-1}\left(\alpha_{0}\right)\right) .
\end{aligned}
$$

From Theorems 3.2 and 3.4 of Hall and Heyde [7] , it is sufficient to show that under $H_{1, n}^{(1)}$,

$$
\begin{aligned}
& \sum_{i=1}^{n} \mathbb{E}_{\theta_{1, n}^{*}}\left[\frac{1}{\sqrt{n}}\left(\xi_{i, 1}^{l_{1}}+\xi_{i, 2}^{l_{1}}\right) \mid \mathcal{G}_{i-1}^{n}\right] \stackrel{P}{\rightarrow} \sum_{j=1}^{p_{1}} I_{a}^{\left(l_{1} j\right)}\left(\alpha_{0} ; \alpha_{0}\right) u_{\alpha, j}, \\
& \sum_{i=1}^{n} \mathbb{E}_{\theta_{1, n}^{*}}\left[\frac{1}{n}\left(\xi_{i, 1}^{l_{1}}+\xi_{i, 2}^{l_{1}}\right)\left(\xi_{i, 1}^{l_{2}}+\xi_{i, 2}^{l_{2}}\right) \mid \mathcal{G}_{i-1}^{n}\right] \stackrel{P}{\rightarrow} I_{a}^{\left(l_{1} l_{2}\right)}\left(\alpha_{0} ; \alpha_{0}\right), \\
& \sum_{i=1}^{n} \mathbb{E}_{\theta_{1, n}^{*}}\left[\frac{1}{\sqrt{n}}\left(\xi_{i, 1}^{l_{1}}+\xi_{i, 2}^{l_{1}}\right) \mid \mathcal{G}_{i-1}^{n}\right] \mathbb{E}_{\theta_{1, n}^{*}}\left[\frac{1}{\sqrt{n}}\left(\xi_{i, 1}^{l_{2}}+\xi_{i, 2}^{l_{2}}\right) \mid \mathcal{G}_{i-1}^{n}\right] \stackrel{P}{\rightarrow} 0, \\
& \sum_{i=1}^{n} \mathbb{E}_{\theta_{1, n}^{*}}\left[\frac{1}{n^{2}}\left(\xi_{i, 1}^{l_{1}}+\xi_{i, 2}^{l_{1}}\right)^{4} \mid \mathcal{G}_{i-1}^{n}\right] \stackrel{P}{\rightarrow} 0,
\end{aligned}
$$

where $l_{1}, l_{2}=1, \ldots, p_{1}$.

Proof of (32). From the Itô-Taylor expansion and Lemma 7 of Kessler 9], we have

$$
\mathbb{E}_{\theta_{1, n}^{*}}\left[\left(X_{i, p}-X_{i-1, p}\right)\left(X_{i, q}-X_{i-1, q}\right) \mid \mathcal{G}_{i-1}^{n}\right]=h_{n} S_{i-1, p q}\left(\alpha_{1, n}^{*}\right)+R_{i-1}\left(h_{n}^{2}\right),
$$

and it holds

$$
\begin{aligned}
\mathbb{E}_{\theta_{1, n}^{*}}\left[\xi_{i, 1}^{l_{1}} \mid \mathcal{G}_{i-1}^{n}\right] & =\frac{1}{2 h_{n}} \sum_{p, q}\left(S^{-1}\left(\partial_{\alpha_{l_{1}}} S\right) S^{-1}\right)_{i-1, p q}\left(\alpha_{0}\right) \mathbb{E}_{\theta_{1, n}^{*}}\left[\left(X_{i, p}-X_{i-1, p}\right)\left(X_{i, q}-X_{i-1, q}\right) \mid \mathcal{G}_{i-1}^{n}\right] \\
& =\frac{1}{2} \sum_{p, q}\left(S^{-1}\left(\partial_{\alpha_{l_{1}}} S\right) S^{-1}\right)_{i-1, p q}\left(\alpha_{0}\right)\left(S_{i-1, p q}\left(\alpha_{1, n}^{*}\right)+R_{i-1}\left(h_{n}\right)\right) \\
& =\frac{1}{2} \sum_{p, q}\left(S^{-1}\left(\partial_{\alpha_{l_{1}}} S\right) S^{-1}\right)_{i-1, p q}\left(\alpha_{0}\right) S_{i-1, p q}\left(\alpha_{1, n}^{*}\right)+R_{i-1}\left(h_{n}\right) .
\end{aligned}
$$


By Taylor's theorem, we obtain

$$
\begin{aligned}
S_{i-1, p q}\left(\alpha_{1, n}^{*}\right)= & S_{i-1, p q}\left(\alpha_{0}\right)+\left(\partial_{\alpha} S\right)_{i-1, p q}\left(\alpha_{0}\right)\left(\alpha_{1, n}^{*}-\alpha_{0}\right) \\
& +\left(\alpha_{1, n}^{*}-\alpha_{0}\right)^{\top}\left(\int_{0}^{1} u\left(\partial_{\alpha}^{2} S\right)_{i-1, p q}\left(\alpha_{0}+u\left(\alpha_{1, n}^{*}-\alpha_{0}\right)\right) d u\right)\left(\alpha_{1, n}^{*}-\alpha_{0}\right) \\
= & S_{i-1, p q}\left(\alpha_{0}\right)+\frac{1}{\sqrt{n}}\left(\partial_{\alpha} S\right)_{i-1, p q}\left(\alpha_{0}\right) u_{\alpha} \\
& +\frac{1}{n} u_{\alpha}^{\top}\left(\int_{0}^{1} u\left(\partial_{\alpha}^{2} S\right)_{i-1, p q}\left(\alpha_{0}+u\left(\alpha_{1, n}^{*}-\alpha_{0}\right)\right) d u\right) u_{\alpha} \\
= & S_{i-1, p q}\left(\alpha_{0}\right)+\frac{1}{\sqrt{n}}\left(\partial_{\alpha} S\right)_{i-1, p q}\left(\alpha_{0}\right) u_{\alpha}+\frac{1}{n} R_{i-1}(1) .
\end{aligned}
$$

Note that $\frac{h_{n}}{\frac{1}{n}}=n h_{n}>1$ and $R_{i-1}\left(h_{n}\right)+\frac{1}{n} R_{i-1}(1)=R_{i-1}\left(h_{n}\right)$, it follows from 36 that

$$
\begin{aligned}
\mathbb{E}_{\theta_{1, n}^{*}}\left[\xi_{i, 1}^{l_{1}} \mid \mathcal{G}_{i-1}^{n}\right]= & \frac{1}{2} \sum_{p, q}\left(S^{-1}\left(\partial_{\alpha_{l_{1}}} S\right) S^{-1}\right)_{i-1, p q}\left(\alpha_{0}\right) S_{i-1, p q}\left(\alpha_{0}\right) \\
& +\frac{1}{2 \sqrt{n}} \sum_{p, q}\left(S^{-1}\left(\partial_{\alpha_{l_{1}}} S\right) S^{-1}\right)_{i-1, p q}\left(\alpha_{0}\right)\left(\partial_{\alpha} S\right)_{i-1, p q}\left(\alpha_{0}\right) u_{\alpha}+R_{i-1}\left(h_{n}\right) \\
= & \frac{1}{2} \operatorname{tr}\left(\left(S^{-1}\left(\partial_{\alpha_{l_{1}}} S\right) S^{-1} S\right)_{i-1}\left(\alpha_{0}\right)\right) \\
& +\frac{1}{2 \sqrt{n}} \sum_{p, q}\left(S^{-1}\left(\partial_{\alpha_{l_{1}}} S\right) S^{-1}\right)_{i-1, p q}\left(\alpha_{0}\right)\left(\sum_{j=1}^{p_{1}}\left(\partial_{\alpha_{j}} S\right)_{i-1, p q}\left(\alpha_{0}\right)\right) u_{\alpha, j} \\
& +R_{i-1}\left(h_{n}\right) \\
= & \frac{1}{2} \operatorname{tr}\left(\left(S^{-1}\left(\partial_{\alpha_{l_{1}}} S\right)\right)_{i-1}\left(\alpha_{0}\right)\right) \\
& +\frac{1}{2 \sqrt{n}} \sum_{j=1}^{p_{1}} \operatorname{tr}\left(\left(S^{-1}\left(\partial_{\alpha_{l_{1}}} S\right) S^{-1}\left(\partial_{\alpha_{j}} S\right)\right)_{i-1}\left(\alpha_{0}\right)\right) u_{\alpha, j}+R_{i-1}\left(h_{n}\right) .
\end{aligned}
$$

On the other hand, we have

$$
\mathbb{E}_{\theta_{1, n}^{*}}\left[\xi_{i, 2}^{l_{1}} \mid \mathcal{G}_{i-1}^{n}\right]=-\frac{1}{2} \operatorname{tr}\left(\left(S^{-1} \partial_{\alpha_{l_{1}}} S\right)_{i-1}\left(\alpha_{0}\right)\right) .
$$

Hence, it follows from Lemma 8 of Kessler [9] that under $H_{0, n}^{(1)}$,

$$
\begin{aligned}
\sum_{i=1}^{n} \mathbb{E}_{\theta_{1, n}^{*}}\left[\frac{1}{\sqrt{n}}\left(\xi_{i, 1}^{l_{1}}+\xi_{i, 2}^{l_{1}}\right) \mid \mathcal{G}_{i-1}^{n}\right]= & \frac{1}{n} \sum_{i=1}^{n}\left\{\frac{1}{2} \sum_{j=1}^{p_{1}} \operatorname{tr}\left(\left(S^{-1}\left(\partial_{\alpha_{1}} S\right) S^{-1}\left(\partial_{\alpha_{j}} S\right)\right)_{i-1}\left(\alpha_{0}\right)\right) u_{\alpha, j}\right\} \\
& +\frac{1}{n} \sum_{i=1}^{n} R_{i-1}\left(\sqrt{n h_{n}^{2}}\right) \\
& \stackrel{P}{\rightarrow} \frac{1}{2} \sum_{i=1}^{p_{1}} \int \operatorname{tr}\left(\left(S^{-1}\left(\partial_{\alpha_{l_{1}}} S\right) S^{-1}\left(\partial_{\alpha_{j}} S\right)\right)\left(x, \alpha_{0}\right)\right) \mu_{\theta_{0}}(d x) u_{\alpha, j} \\
= & \sum_{i=1}^{p_{1}} I_{a}^{\left(l_{1} j\right)}\left(\alpha_{0} ; \alpha_{0}\right) u_{\alpha, j} .
\end{aligned}
$$

By setting

$$
Q_{n}:=\sum_{i=1}^{n} \mathbb{E}_{\theta_{1, n}^{*}}\left[\frac{1}{\sqrt{n}}\left(\xi_{i, 1}^{l_{1}}+\xi_{i, 2}^{l_{1}}\right) \mid \mathcal{G}_{i-1}^{n}\right]-\sum_{i=1}^{p_{1}} I_{a}^{\left(l_{1} j\right)}\left(\alpha_{0} ; \alpha_{0}\right) u_{\alpha, j}
$$


the above convergence implies that for all $\varepsilon>0$,

$$
\lim _{n \rightarrow \infty} P_{\theta_{0}}\left(\left|Q_{n}\right| \geq \varepsilon\right)=0 .
$$

Hence it follows from $\mathbf{C} 1$ that

$$
\lim _{n \rightarrow \infty} P_{\theta_{1, n}^{*}}\left(\left|Q_{n}\right| \geq \varepsilon\right)=0,
$$

which implies $(32)$.

Proof of $(33)$. From the Itô-Taylor expansion, it holds that for $1 \leq p, q, r, s \leq p_{1}$,

$$
\begin{aligned}
\mathbb{E}_{\theta_{1, n}^{*}} & {\left[\left(X_{i, p}-X_{i-1, p}\right)\left(X_{i, q}-X_{i-1, q}\right)\left(X_{i, r}-X_{i-1, r}\right)\left(X_{i, s}-X_{i-1, s}\right) \mid \mathcal{G}_{i-1}^{n}\right] } \\
= & h_{n}^{2}\left(S_{i-1, p q}\left(\alpha_{1, n}^{*}\right) S_{i-1, r s}\left(\alpha_{1, n}^{*}\right)+S_{i-1, p r}\left(\alpha_{1, n}^{*}\right) S_{i-1, q s}\left(\alpha_{1, n}^{*}\right)+S_{i-1, p s}\left(\alpha_{1, n}^{*}\right) S_{i-1, q r}\left(\alpha_{1, n}^{*}\right)\right) \\
& +R_{i-1}\left(h_{n}^{3}\right) .
\end{aligned}
$$

Noting that $\frac{h_{n}}{\frac{1}{\sqrt{n}}}=\sqrt{n h_{n}^{2}}<1$, we obtain

$$
\begin{aligned}
\mathbb{E}_{\theta_{1, n}^{*}}\left[\xi_{i, 1}^{l_{1}} \xi_{i, 1}^{l_{2}} \mid \mathcal{G}_{i-1}^{n}\right]= & \frac{1}{4 h_{n}^{2}} \sum_{p, q, r, s}\left(\partial_{\alpha_{l_{1}}} S^{-1}\right)_{i-1, p q}\left(\alpha_{0}\right)\left(\partial_{\alpha_{l_{2}}} S^{-1}\right)_{i-1, r s}\left(\alpha_{0}\right) \\
& +\mathbb{E}_{\theta_{1, n}^{*}}\left[\left(X_{i, p}-X_{i-1, p}\right)\left(X_{i, q}-X_{i-1, q}\right)\left(X_{i, r}-X_{i-1, r}\right)\left(X_{i, s}-X_{i-1, s}\right) \mid \mathcal{G}_{i-1}^{n}\right] \\
= & \frac{1}{4} \sum_{p, q, r, s}\left(\partial_{\alpha_{l_{1}}} S^{-1}\right)_{i-1, p q}\left(\alpha_{0}\right)\left(\partial_{\alpha_{l_{2}}} S^{-1}\right)_{i-1, r s}\left(\alpha_{0}\right) \\
& \times\left(S_{i-1, p q}\left(\alpha_{1, n}^{*}\right) S_{i-1, r s}\left(\alpha_{1, n}^{*}\right)+S_{i-1, p r}\left(\alpha_{1, n}^{*}\right) S_{i-1, q s}\left(\alpha_{1, n}^{*}\right)\right. \\
& \left.+S_{i-1, p s}\left(\alpha_{1, n}^{*}\right) S_{i-1, q r}\left(\alpha_{1, n}^{*}\right)\right) \\
& +R_{i-1}\left(h_{n}\right) \\
= & \frac{1}{4} \sum_{p, q, r, s}\left(\partial_{\alpha_{l_{1}}} S^{-1}\right)_{i-1, p q}\left(\alpha_{0}\right)\left(\partial_{\alpha_{l_{2}}} S^{-1}\right)_{i-1, r s}\left(\alpha_{0}\right) \\
& \times\left(S_{i-1, p q}\left(\alpha_{0}\right) S_{i-1, r s}\left(\alpha_{0}\right)+S_{i-1, p r}\left(\alpha_{0}\right) S_{i-1, q s}\left(\alpha_{0}\right)\right. \\
& \left.+S_{i-1, p s}\left(\alpha_{0}\right) S_{i-1, q r}\left(\alpha_{0}\right)\right) \\
& +\frac{1}{\sqrt{n}} R_{i-1}(1) .
\end{aligned}
$$

Since

$$
\begin{aligned}
& \sum_{p, q, r, s}\left(\partial_{\alpha_{l_{1}}} S^{-1}\right)_{i-1, p q}\left(\alpha_{0}\right)\left(\partial_{\alpha_{l_{2}}} S^{-1}\right)_{i-1, r s}\left(\alpha_{0}\right) S_{i-1, p q}\left(\alpha_{0}\right) S_{i-1, r s}\left(\alpha_{0}\right) \\
& \quad=\operatorname{tr}\left(\left(\left(\partial_{\alpha_{l_{1}}} S^{-1}\right) S\right)_{i-1}\left(\alpha_{0}\right)\right) \operatorname{tr}\left(\left(\left(\partial_{\alpha_{l_{2}}} S^{-1}\right) S\right)_{i-1}\left(\alpha_{0}\right)\right), \\
& \sum_{p, q, r, s}\left(\partial_{\alpha_{l_{1}}} S^{-1}\right)_{i-1, p q}\left(\alpha_{0}\right)\left(\partial_{\alpha_{l_{2}}} S^{-1}\right)_{i-1, r s}\left(\alpha_{0}\right) S_{i-1, p r}\left(\alpha_{0}\right) S_{i-1, q s}\left(\alpha_{0}\right) \\
& \quad=\sum_{p, q, r, s}\left(\partial_{\alpha_{l_{1}}} S^{-1}\right)_{i-1, p q}\left(\alpha_{0}\right)\left(\partial_{\alpha_{l_{2}}} S^{-1}\right)_{i-1, r s}\left(\alpha_{0}\right) S_{i-1, p s}\left(\alpha_{0}\right) S_{i-1, q r}\left(\alpha_{0}\right) \\
& \quad=\operatorname{tr}\left(\left(\left(\partial_{\alpha_{l_{1}}} S^{-1}\right) S\left(\partial_{\alpha_{l_{2}}} S^{-1}\right) S\right)_{i-1}\left(\alpha_{0}\right)\right),
\end{aligned}
$$

it follows from (37) that

$$
\begin{aligned}
\mathbb{E}_{\theta_{1, n}^{*}}\left[\xi_{i, 1}^{l_{1}} \xi_{i, 1}^{l_{2}} \mid \mathcal{G}_{i-1}^{n}\right]= & \frac{1}{4} \operatorname{tr}\left(\left(\left(\partial_{\alpha_{l_{1}}} S^{-1}\right) S\right)_{i-1}\left(\alpha_{0}\right)\right) \operatorname{tr}\left(\left(\left(\partial_{\alpha_{l_{2}}} S^{-1}\right) S\right)_{i-1}\left(\alpha_{0}\right)\right) \\
& +\frac{1}{2} \operatorname{tr}\left(\left(\left(\partial_{\alpha_{l_{1}}} S^{-1}\right) S\left(\partial_{\alpha_{l_{2}}} S^{-1}\right) S\right)_{i-1}\left(\alpha_{0}\right)\right)+\frac{1}{\sqrt{n}} R_{i-1}(1) .
\end{aligned}
$$


Moreover, we have

$$
\begin{aligned}
\mathbb{E}_{\theta_{1, n}^{*}}\left[\xi_{i, 2}^{l_{1}} \xi_{i, 2}^{l_{2}} \mid \mathcal{G}_{i-1}^{n}\right]= & \frac{1}{4} \operatorname{tr}\left(\left(S^{-1} \partial_{\alpha_{l_{1}}} S\right)_{i-1}\left(\alpha_{0}\right)\right) \operatorname{tr}\left(\left(S^{-1} \partial_{\alpha_{l_{2}}} S\right)_{i-1}\left(\alpha_{0}\right)\right) \\
\mathbb{E}_{\theta_{1, n}^{*}}\left[\xi_{i, 1}^{l_{1}} \xi_{i, 2}^{l_{2}} \mid \mathcal{G}_{i-1}^{n}\right]= & -\frac{1}{4 h_{n}} \operatorname{tr}\left(\left(S^{-1} \partial_{\alpha_{l_{2}}} S\right)_{i-1}\left(\alpha_{0}\right)\right) \sum_{p, q}\left(S^{-1}\left(\partial_{\alpha_{l_{1}}} S\right) S^{-1}\right)_{i-1, p q}\left(\alpha_{0}\right) \\
& \times \mathbb{E}_{\theta_{1, n}^{*}}\left[\left(X_{i, p}-X_{i-1, p}\right)\left(X_{i, q}-X_{i-1, q}\right) \mid \mathcal{G}_{i-1}^{n}\right] \\
= & -\frac{1}{4} \operatorname{tr}\left(\left(S^{-1} \partial_{\alpha_{l_{2}}} S\right)_{i-1}\left(\alpha_{0}\right)\right) \sum_{p, q}\left(S^{-1}\left(\partial_{\alpha_{l_{1}}} S\right) S^{-1}\right)_{i-1, p q}\left(\alpha_{0}\right) S_{i-1, p q}\left(\alpha_{0}\right) \\
& +\frac{1}{\sqrt{n}} R_{i-1}(1) \\
= & -\frac{1}{4} \operatorname{tr}\left(\left(S^{-1} \partial_{\alpha_{l_{2}}} S\right)_{i-1}\left(\alpha_{0}\right)\right) \operatorname{tr}\left(\left(S^{-1} \partial_{\alpha_{l_{1}}} S\right)_{i-1}\left(\alpha_{0}\right)\right)+\frac{1}{\sqrt{n}} R_{i-1}(1) .
\end{aligned}
$$

Therefore, it holds that under $H_{0, n}^{(1)}$,

$$
\begin{aligned}
\sum_{i=1}^{n} \mathbb{E}_{\theta_{1, n}^{*}}\left[\frac{1}{n}\left(\xi_{i, 1}^{l_{1}}+\xi_{i, 2}^{l_{1}}\right)\left(\xi_{i, 1}^{l_{2}}+\xi_{i, 2}^{l_{2}}\right) \mid \mathcal{G}_{i-1}^{n}\right]= & \frac{1}{n} \sum_{i=1}^{n} \frac{1}{2} \operatorname{tr}\left(\left(\left(\partial_{\alpha_{l_{1}}} S^{-1}\right) S\left(\partial_{\alpha_{l_{2}}} S^{-1}\right) S\right)_{i-1}\left(\alpha_{0}\right)\right) \\
& +\frac{1}{n} \sum_{i=1}^{n} \frac{1}{\sqrt{n}} R_{i-1}(1) \\
& \stackrel{P}{\rightarrow} \frac{1}{2} \int \operatorname{tr}\left(\left(\left(\partial_{\alpha_{l_{1}}} S^{-1}\right) S\left(\partial_{\alpha_{l_{2}}} S^{-1}\right) S\right)\left(x, \alpha_{0}\right)\right) \mu_{\theta_{0}}(d x) \\
& =\frac{1}{2} \int \operatorname{tr}\left(\left(S^{-1}\left(\partial_{\alpha_{l_{1}}} S\right) S^{-1}\left(\partial_{\alpha_{l_{2}}} S\right)\right)\left(x, \alpha_{0}\right)\right) \mu_{\theta_{0}}(d x) \\
& =I_{a}^{\left(l_{1} l_{2}\right)}\left(\alpha_{0} ; \alpha_{0}\right) .
\end{aligned}
$$

From C1, this convergence also holds under $H_{1, n}^{(1)}$.

Proof of (34). From the proof of 32 , we have

$$
\begin{aligned}
& \mathbb{E}_{\theta_{1, n}^{*}}\left[\frac{1}{\sqrt{n}}\left(\xi_{i, 1}^{l_{1}}+\xi_{i, 2}^{l_{1}}\right) \mid \mathcal{G}_{i-1}^{n}\right]=\frac{1}{n} R_{i-1}(1), \\
& \mathbb{E}_{\theta_{1, n}^{*}}\left[\frac{1}{\sqrt{n}}\left(\xi_{i, 1}^{l_{2}}+\xi_{i, 2}^{l_{2}}\right) \mid \mathcal{G}_{i-1}^{n}\right]=\frac{1}{n} R_{i-1}(1) .
\end{aligned}
$$

Therefore, it holds that under $H_{0, n}^{(1)}$,

$$
\sum_{i=1}^{n} \mathbb{E}_{\theta_{1, n}^{*}}\left[\frac{1}{\sqrt{n}}\left(\xi_{i, 1}^{l_{1}}+\xi_{i, 2}^{l_{1}}\right) \mid \mathcal{G}_{i-1}^{n}\right] \mathbb{E}_{\theta_{1, n}^{*}}\left[\frac{1}{\sqrt{n}}\left(\xi_{i, 1}^{l_{2}}+\xi_{i, 2}^{l_{2}}\right) \mid \mathcal{G}_{i-1}^{n}\right]=\frac{1}{n} \sum_{i=1}^{n} \frac{1}{n} R_{i-1}(1) \stackrel{P}{\rightarrow} 0
$$

and this also holds under $H_{1, n}^{(1)}$.

Proof of 35 . It is easy to show

$$
\mathbb{E}_{\theta_{1, n}^{*}}\left[\frac{1}{n^{2}}\left(\xi_{i, 1}^{l_{1}}+\xi_{i, 2}^{l_{1}}\right)^{4} \mid \mathcal{G}_{i-1}^{n}\right] \leq \frac{2^{3}}{n^{2}}\left(\mathbb{E}_{\theta_{1, n}^{*}}\left[\left(\xi_{i, 1}^{l_{1}}\right)^{4} \mid \mathcal{G}_{i-1}^{n}\right]+\mathbb{E}_{\theta_{1, n}^{*}}\left[\left(\xi_{i, 2}^{l_{1}}\right)^{4} \mid \mathcal{G}_{i-1}^{n}\right]\right)
$$


We can evaluate

$$
\begin{aligned}
\mathbb{E}_{\theta_{1, n}^{*}}\left[\left(\xi_{i, 1}^{l_{1}}\right)^{4} \mid \mathcal{G}_{i-1}^{n}\right] & =\frac{1}{16 h_{n}^{4}} \mathbb{E}_{\theta_{1, n}^{*}}\left[\left(\sum_{p, q}\left(\partial_{\alpha_{l_{1}}} S^{-1}\right)_{i-1, p q}\left(\alpha_{0}\right)\right.\right. \\
& \left.\left.\times\left(X_{i, p}-X_{i-1, q}\right)\left(X_{i, q}-X_{i-1, q}\right)\right)^{4} \mid \mathcal{G}_{i-1}^{n}\right] \\
& \leq \frac{C}{16 h_{n}^{4}}\left(1+\left|X_{t_{i-1}^{n}}\right|\right)^{C} \sum_{1 \leq p_{1}, \ldots p_{8} \leq d} \mathbb{E}_{\theta_{1, n}^{*}}\left[\prod_{j=1}^{8}\left(X_{i, p_{j}}-X_{i-1, p_{j}}\right) \mid \mathcal{G}_{i-1}^{n}\right] \\
& \leq R_{i-1}\left(h_{n}^{-4}\right) \sum_{1 \leq p_{1}, \ldots p_{8} \leq d} \prod_{j=1}^{8}\left(\mathbb{E}_{\theta_{1, n}^{*}}\left[\left(X_{i, p_{j}}-X_{i-1, p_{j}}\right)^{8} \mid \mathcal{G}_{i-1}^{n}\right]\right)^{\frac{1}{8}} \\
& \leq d^{8} R_{i-1}\left(h_{n}^{-4}\right) \mathbb{E}_{\theta_{1, n}^{*}}\left[\left|X_{t_{i}^{n}}-X_{t_{i-1}}^{n}\right|^{8} \mid \mathcal{G}_{i-1}^{n}\right] \\
& \leq R_{i-1}(1), \\
\mathbb{E}_{\theta_{1, n}^{*}}\left[\left(\xi_{i, 2}^{l_{1}}\right)^{4} \mid \mathcal{G}_{i-1}^{n}\right] & =\frac{1}{16}\left(\operatorname{tr}\left(\left(S^{-1} \partial_{\alpha_{l_{1}}} S\right)_{i-1}\left(\alpha_{0}\right)\right)\right)^{4} \\
& =R_{i-1}(1) .
\end{aligned}
$$

Hence

$$
\sum_{i=1}^{n} \frac{2^{3}}{n^{2}}\left(\mathbb{E}_{\theta_{1, n}^{*}}\left[\left(\xi_{i, 1}^{l_{1}}\right)^{4} \mid \mathcal{G}_{i-1}^{n}\right]+\mathbb{E}_{\theta_{1, n}^{*}}\left[\left(\xi_{i, 2}^{l_{1}}\right)^{4} \mid \mathcal{G}_{i-1}^{n}\right]\right)=\frac{1}{n} \sum_{i=1}^{n} \frac{1}{n} R_{i-1}(1) \stackrel{P}{\rightarrow} 0
$$

and

$$
\sum_{i=1}^{n} \mathbb{E}_{\theta_{1, n}^{*}}\left[\frac{1}{n^{2}}\left(\xi_{i, 1}^{l_{1}}+\xi_{i, 2}^{l_{1}}\right)^{4} \mid \mathcal{G}_{i-1}^{n}\right] \stackrel{P}{\rightarrow} 0 \quad\left(\text { under } H_{0, n}^{(1)}\right)
$$

From C1, the last convergence also holds under $H_{1, n}^{(1)}$. By using 32 - 35 , we get 31 .

Next we prove (i). It follows from Taylor's theorem that

$$
-\frac{1}{\sqrt{n}} \partial_{\alpha} U_{n}^{(1)}\left(\alpha_{0}\right)=\int_{0}^{1} \frac{1}{n} \partial_{\alpha}^{2} U_{n}^{(1)}\left(\alpha_{0}+u\left(\hat{\alpha}_{n}-\alpha_{0}\right)\right) d u \sqrt{n}\left(\hat{\alpha}_{n}-\alpha_{0}\right) .
$$

It holds

$$
\int_{0}^{1} \frac{1}{n} \partial_{\alpha}^{2} U_{n}^{(1)}\left(\alpha_{0}+u\left(\hat{\alpha}_{n}-\alpha_{0}\right)\right) d u \stackrel{P}{\rightarrow}-I_{a}\left(\alpha_{0} ; \alpha_{0}\right) \quad\left(\text { under } H_{0, n}^{(1)}\right)
$$

which also holds under $H_{1, n}^{(1)}$. By noting that $I_{a}\left(\alpha_{0} ; \alpha_{0}\right)$ is non-singular, it follows from Slutsky's theorem that

$$
\sqrt{n}\left(\hat{\alpha}_{n}-\alpha_{0}\right) \stackrel{d}{\rightarrow} I_{a}^{-1}\left(\alpha_{0} ; \alpha_{0}\right) L_{1}=Y_{1}^{\prime} \quad\left(\text { under } H_{1, n}^{(1)}\right) .
$$

This completes the proof of (i).

(ii) First of all, we will prove that

$$
\frac{1}{\sqrt{n h_{n}}} \partial_{\beta} U_{n}^{(2)}\left(\beta_{0} \mid \alpha_{0}\right) \stackrel{d}{\rightarrow} L_{2} \sim N\left(I_{b}\left(\theta_{0} ; \theta_{0}\right) u_{\beta}, I_{b}\left(\theta_{0} ; \theta_{0}\right)\right) \quad\left(\text { under } H_{1, n}^{(2)}\right) .
$$

Letting $b\left(X_{t_{i-1}^{n}}, \beta\right)=\left(b_{i-1,1}(\beta), \ldots, b_{i-1, d}(\beta)\right)^{\top}$ and $u_{\beta}=\left(u_{\beta, 1}, \ldots, u_{\beta, p_{2}}\right)^{\top}$, one has that

$$
\begin{aligned}
U_{n}^{(2)}(\beta \mid \bar{\alpha}) & =-\frac{1}{2} \sum_{i=1}^{n}\left\{h_{n}^{-1} S^{-1}\left(X_{t_{i-1}^{n}}, \bar{\alpha}\right)\left[\left(X_{t_{i}^{n}}-X_{t_{i-1}^{n}}-h_{n} b\left(X_{t_{i-1}^{n}}, \beta\right)\right)^{\otimes 2}\right]\right\} \\
& =-\frac{1}{2 h_{n}} \sum_{i=1}^{n} \sum_{p, q} S_{i-1, p q}^{-1}(\bar{\alpha})\left(X_{i, p}-X_{i-1, p}-h_{n} b_{i-1, p}(\beta)\right)\left(X_{i, q}-X_{i-1, q}-h_{n} b_{i-1, q}(\beta)\right) .
\end{aligned}
$$


For $m_{1}=1, \ldots, p_{2}$, we have

$$
\begin{aligned}
& \partial_{\beta_{m_{1}}} U_{n}^{(2)}\left(\beta_{0} \mid \alpha_{0}\right)=\sum_{i=1}^{n} \eta_{i, 1}^{m_{1}} \\
& \eta_{i, 1}^{m_{1}}=\sum_{p, q} S_{i-1, p q}^{-1}\left(\alpha_{0}\right)\left(\partial_{\beta_{m_{1}}} b\right)_{i-1, q}\left(\beta_{0}\right)\left(X_{i, p}-X_{i-1, p}-h_{n} b_{i-1, p}\left(\beta_{0}\right)\right) .
\end{aligned}
$$

From Theorems 3.2 and 3.4 of Hall and Heyde [7] and $\mathbf{C 1}$, it is sufficient to show that under $H_{0, n}^{(2)}$,

$$
\begin{aligned}
& \sum_{i=1}^{n} \mathbb{E}_{\theta_{1, n}^{*}}\left[\frac{1}{\sqrt{n h_{n}}} \eta_{i, 1}^{m_{1}} \mid \mathcal{G}_{i-1}^{n}\right] \stackrel{P}{\rightarrow} \sum_{j=1}^{p_{2}} I_{b}^{\left(m_{1} j\right)}\left(\theta_{0} ; \theta_{0}\right) u_{\beta, j}, \\
& \sum_{i=1}^{n} \mathbb{E}_{\theta_{1, n}^{*}}\left[\frac{1}{n h_{n}} \eta_{i, 1}^{m_{1}} \eta_{i, 1}^{m_{2}} \mid \mathcal{G}_{i-1}^{n}\right] \stackrel{P}{\rightarrow} I_{b}^{\left(m_{1} m_{2}\right)}\left(\theta_{0} ; \theta_{0}\right), \\
& \sum_{i=1}^{n} \mathbb{E}_{\theta_{1, n}^{*}}\left[\frac{1}{\sqrt{n h_{n}}} \eta_{i, 1}^{m_{1}} \mid \mathcal{G}_{i-1}^{n}\right] \mathbb{E}_{\theta_{1, n}^{*}}\left[\frac{1}{\sqrt{n h_{n}}} \eta_{i, 1}^{m_{2}} \mid \mathcal{G}_{i-1}^{n}\right] \stackrel{P}{\rightarrow} 0, \\
& \sum_{i=1}^{n} \mathbb{E}_{\theta_{1, n}^{*}}\left[\frac{1}{\left(n h_{n}\right)^{2}}\left(\eta_{i, 1}^{m_{1}}\right)^{4} \mid \mathcal{G}_{i-1}^{n}\right] \stackrel{P}{\rightarrow} 0,
\end{aligned}
$$

where $m_{1}, m_{2}=1, \ldots, p_{2}$.

Proof of (39). By the Itô-Taylor expansion, we have

$$
\mathbb{E}_{\theta_{1, n}^{*}}\left[X_{i, p}-X_{i-1, p} \mid \mathcal{G}_{i-1}^{n}\right]=h_{n} b_{i-1, p}\left(\beta_{1, n}^{*}\right)+R_{i-1}\left(h_{n}^{2}\right),
$$

and

$$
\begin{aligned}
\mathbb{E}_{\theta_{1, n}^{*}}\left[\eta_{i, 1}^{m_{1}} \mid \mathcal{G}_{i-1}^{n}\right] & =\sum_{p, q} S_{i-1, p q}^{-1}\left(\alpha_{0}\right)\left(\partial_{\beta_{m_{1}}} b\right)_{i-1, q}\left(\beta_{0}\right)\left(\mathbb{E}_{\theta_{1, n}^{*}}\left[X_{i, p}-X_{i-1, p} \mid \mathcal{G}_{i-1}^{n}\right]-h_{n} b_{i-1, p}\left(\beta_{0}\right)\right) \\
& =h_{n} \sum_{p, q} S_{i-1, p q}^{-1}\left(\alpha_{0}\right)\left(\partial_{\beta_{m_{1}}} b\right)_{i-1, q}\left(\beta_{0}\right)\left(b_{i-1, p}\left(\beta_{1, n}^{*}\right)-b_{i-1, p}\left(\beta_{0}\right)\right)+R_{i-1}\left(h_{n}^{2}\right) .
\end{aligned}
$$

Since it follows from Taylor's theorem that

$$
\begin{aligned}
b_{i-1, p}\left(\beta_{1, n}^{*}\right)-b_{i-1, p}\left(\beta_{0}\right)= & \left(\partial_{\beta} b\right)_{i-1, p}\left(\beta_{0}\right)\left(\beta_{1, n}^{*}-\beta_{0}\right) \\
& +\left(\beta_{1, n}^{*}-\beta_{0}\right)^{\top}\left(\int_{0}^{1} u\left(\partial_{\beta}^{2} b\right)_{i-1, p}\left(\beta_{0}+u\left(\beta_{1, n}^{*}-\beta_{0}\right)\right) d u\right)\left(\beta_{1, n}^{*}-\beta_{0}\right) \\
= & \frac{1}{\sqrt{n h_{n}}}\left(\partial_{\beta} b\right)_{i-1, p}\left(\beta_{0}\right) u_{\beta}+\frac{1}{n h_{n}} R_{i-1}(1),
\end{aligned}
$$

(43) implies that

$$
\begin{aligned}
& \mathbb{E}_{\theta_{1, n}^{*}}\left[\eta_{i, 1}^{m_{1}} \mid \mathcal{G}_{i-1}^{n}\right]=\sqrt{\frac{h_{n}}{n}} \sum_{p, q} S_{i-1, p q}^{-1}\left(\alpha_{0}\right)\left(\partial_{\beta_{m_{1}}} b\right)_{i-1, q}\left(\beta_{0}\right)\left(\partial_{\beta} b\right)_{i-1, p}\left(\beta_{0}\right) u_{\beta}+\frac{1}{n} R_{i-1}(1) \\
& =\sqrt{\frac{h_{n}}{n}} \sum_{j=1}^{p_{2}} \sum_{p, q} S_{i-1, p q}^{-1}\left(\alpha_{0}\right)\left(\partial_{\beta_{m_{1}}} b\right)_{i-1, q}\left(\beta_{0}\right)\left(\partial_{\beta_{j}} b\right)_{i-1, p}\left(\beta_{0}\right) u_{\beta, j}+\frac{1}{n} R_{i-1}(1) \\
& =\sqrt{\frac{h_{n}}{n}} \sum_{j=1}^{p_{2}}\left(\left(\partial_{\beta_{m_{1}}} b\right)_{i-1}\left(\beta_{0}\right)\right)^{\top} S_{i-1}^{-1}\left(\alpha_{0}\right)\left(\partial_{\beta_{j}} b\right)_{i-1}\left(\beta_{0}\right) u_{\beta, j}+\frac{1}{n} R_{i-1}(1) .
\end{aligned}
$$


Therefore, it follows from Lemma 8 of Kessler 9 ] that under $H_{0, n}^{(1)}$,

$$
\begin{aligned}
\sum_{i=1}^{n} \mathbb{E}_{\theta_{1, n}^{*}}\left[\frac{1}{\sqrt{n h_{n}}} \eta_{i, 1}^{m_{1}} \mid \mathcal{G}_{i-1}^{n}\right]= & \frac{1}{n} \sum_{i=1}^{n} \sum_{j=1}^{p_{2}}\left(\left(\partial_{\beta_{m_{1}}} b\right)_{i-1}\left(\beta_{0}\right)\right)^{\top} S_{i-1}^{-1}\left(\alpha_{0}\right)\left(\partial_{\beta_{j}} b\right)_{i-1}\left(\beta_{0}\right) u_{\beta, j} \\
& +\frac{1}{n} \sum_{i=1}^{n} \frac{1}{\sqrt{n h_{n}}} R_{i-1}(1) \\
& \stackrel{P}{\rightarrow} \sum_{j=1}^{p_{2}} \int\left(\partial_{\beta_{m_{1}}} b\left(x, \beta_{0}\right)\right)^{\top} S^{-1}\left(x, \alpha_{0}\right)\left(\partial_{\beta_{j}} b\left(x, \beta_{0}\right)\right) \mu_{\theta_{0}}(d x) u_{\beta, j} \\
= & \sum_{j=1}^{p_{2}} I_{b}^{\left(m_{1} j\right)}\left(\theta_{0} ; \theta_{0}\right) u_{\beta, j} .
\end{aligned}
$$

Proof of 40. By the Itô-Taylor expansion, one has that

$$
\mathbb{E}_{\theta_{1, n}^{*}}\left[\left(X_{i, p}-X_{i-1, p}-h_{n} b_{i-1, p}\left(\beta_{0}\right)\right)\left(X_{i, q}-X_{i-1, q}-h_{n} b_{i-1, q}\left(\beta_{0}\right)\right) \mid \mathcal{G}_{i-1}^{n}\right]=h_{n} S_{i-1, p q}\left(\alpha_{0}\right)+\frac{1}{\sqrt{n}} R_{i-1}\left(h_{n}\right) .
$$

Setting $\delta_{p s}:=\left\{\begin{array}{ll}1 & (p=s), \\ 0 & (p \neq s),\end{array}\right.$ we obtain that

$$
\begin{aligned}
\mathbb{E}_{\theta_{1, n}^{*}}\left[\eta_{i, 1}^{m_{1}} \eta_{i, 1}^{m_{2}} \mid \mathcal{G}_{i-1}^{n}\right]= & \sum_{p, q, r, s} S_{i-1, p q}^{-1}\left(\alpha_{0}\right) S_{i-1, r s}^{-1}\left(\alpha_{0}\right)\left(\partial_{\beta_{m_{1}}} b\right)_{i-1, q}\left(\beta_{0}\right)\left(\partial_{\beta_{m_{2}}} b\right)_{i-1, s}\left(\beta_{0}\right) \\
& \times \mathbb{E}_{\theta_{1, n}^{*}}\left[\left(X_{i, p}-X_{i-1, p}-h_{n} b_{i-1, p}\left(\beta_{0}\right)\right)\left(X_{i, r}-X_{i-1, r}-h_{n} b_{i-1, r}\left(\beta_{0}\right)\right) \mid \mathcal{G}_{i-1}^{n}\right] \\
= & h_{n} \sum_{p, q, r, s} S_{i-1, p q}^{-1}\left(\alpha_{0}\right) S_{i-1, r s}^{-1}\left(\alpha_{0}\right) S_{i-1, p r}\left(\alpha_{0}\right) \\
& \times\left(\partial_{\beta_{m_{1}}} b\right)_{i-1, q}\left(\beta_{0}\right)\left(\partial_{\beta_{m_{2}}} b\right)_{i-1, s}\left(\beta_{0}\right)+\frac{1}{\sqrt{n}} R_{i-1}\left(h_{n}\right) \\
= & h_{n} \sum_{p, q, s} S_{i-1, p q}^{-1}\left(\alpha_{0}\right)\left(\sum_{r=1}^{d} S_{i-1, r s}^{-1}\left(\alpha_{0}\right) S_{i-1, r p}\left(\alpha_{0}\right)\right) \\
& \times\left(\partial_{\beta_{m_{1}}} b\right)_{i-1, q}\left(\beta_{0}\right)\left(\partial_{\beta_{m_{2}}} b\right)_{i-1, s}\left(\beta_{0}\right)+\frac{1}{\sqrt{n}} R_{i-1}\left(h_{n}\right) \\
= & h_{n} \sum_{p, q, s} S_{i-1, p q}^{-1}\left(\alpha_{0}\right) \delta_{p s}\left(\partial_{\beta_{m_{1}}} b\right)_{i-1, q}\left(\beta_{0}\right)\left(\partial_{\beta_{m_{2}}} b\right)_{i-1, s}\left(\beta_{0}\right)+\frac{1}{\sqrt{n}} R_{i-1}\left(h_{n}\right) \\
= & h_{n} \sum_{p, q} S_{i-1, p q}^{-1}\left(\alpha_{0}\right)\left(\partial_{\beta_{m_{1}}} b\right)_{i-1, q}\left(\beta_{0}\right)\left(\partial_{\beta_{m_{2}}} b\right)_{i-1, p}\left(\beta_{0}\right)+\frac{1}{\sqrt{n}} R_{i-1}\left(h_{n}\right) \\
= & h_{n}\left(\left(\partial_{\beta_{m_{1}}} b\right)_{i-1}\left(\beta_{0}\right)\right)^{\top} S_{i-1}^{-1}\left(\alpha_{0}\right)\left(\left(\partial_{\beta_{m_{2}}} b\right)_{i-1}\left(\beta_{0}\right)\right)+\frac{1}{\sqrt{n}} R_{i-1}\left(h_{n}\right) .
\end{aligned}
$$

Hence it holds

$$
\begin{aligned}
\sum_{i=1}^{n} \mathbb{E}_{\theta_{1, n}^{*}}\left[\frac{1}{n h_{n}} \eta_{i, 1}^{m_{1}} \eta_{i, 1}^{m_{2}} \mid \mathcal{G}_{i-1}^{n}\right]= & \frac{1}{n} \sum_{i=1}^{n}\left(\left(\partial_{\beta_{m_{1}}} b\right)_{i-1}\left(\beta_{0}\right)\right)^{\top} S_{i-1}^{-1}\left(\alpha_{0}\right)\left(\left(\partial_{\beta_{m_{2}}} b\right)_{i-1}\left(\beta_{0}\right)\right) \\
& +\frac{1}{n} \sum_{i=1}^{n} \frac{1}{\sqrt{n}} R_{i-1}(1) \\
& \stackrel{P}{\rightarrow} \int\left(\partial_{\beta_{m_{1}}} b\left(x, \beta_{0}\right)\right)^{\top} S^{-1}\left(x, \alpha_{0}\right)\left(\partial_{\beta_{m_{2}}} b\left(x, \beta_{0}\right)\right) \mu_{\theta_{0}}(d x) \\
= & I_{b}^{\left(m_{1} m_{2}\right)}\left(\theta_{0} ; \theta_{0}\right) .
\end{aligned}
$$


Proof of 41. From the proof of 39, we have

Hence

$$
\begin{aligned}
& \mathbb{E}_{\theta_{1, n}^{*}}\left[\frac{1}{\sqrt{n h_{n}}} \eta_{i, 1}^{m_{1}} \mid \mathcal{G}_{i-1}^{n}\right]=\frac{1}{n} R_{i-1}(1), \\
& \mathbb{E}_{\theta_{1, n}^{*}}\left[\frac{1}{\sqrt{n h_{n}}} \eta_{i, 1}^{m_{2}} \mid \mathcal{G}_{i-1}^{n}\right]=\frac{1}{n} R_{i-1}(1) .
\end{aligned}
$$

$$
\sum_{i=1}^{n} \mathbb{E}_{\theta_{1, n}^{*}}\left[\frac{1}{\sqrt{n h_{n}}} \eta_{i, 1}^{m_{1}} \mid \mathcal{G}_{i-1}^{n}\right] \mathbb{E}_{\theta_{1, n}^{*}}\left[\frac{1}{\sqrt{n h_{n}}} \eta_{i, 1}^{m_{2}} \mid \mathcal{G}_{i-1}^{n}\right]=\frac{1}{n} \sum_{i=1}^{n} \frac{1}{n} R_{i-1}(1) \stackrel{P}{\rightarrow} 0 .
$$

Proof of 42 . It follows from the Itô-Taylor expansion that

$$
\mathbb{E}_{\theta_{1, n}^{*}}\left[\prod_{j=1}^{4}\left(X_{i, p_{j}}-X_{i-1, p_{j}}-h_{n} b_{i-1, p_{j}}\left(\beta_{0}\right)\right) \mid \mathcal{G}_{i-1}^{n}\right]=R_{i-1}\left(h_{n}^{2}\right) .
$$

Hence we can evaluate

$$
\begin{aligned}
\mathbb{E}_{\theta_{1, n}^{*}}\left[\left(\eta_{i, 1}^{m_{1}}\right)^{4} \mid \mathcal{G}_{i-1}^{n}\right] & =\mathbb{E}_{\theta_{1, n}^{*}}\left[\left(\sum_{p, q} S_{i-1, p q}^{-1}\left(\alpha_{0}\right)\left(\partial_{\beta_{m_{1}}} b\right)_{i-1, q}\left(\beta_{0}\right)\right.\right. \\
& \left.\left.\times\left(X_{i, p}-X_{i-1, p}-h_{n} b_{i-1, p}\left(\beta_{0}\right)\right)\right)^{4} \mid \mathcal{G}_{i-1}^{n}\right] \\
& \leq R_{i-1}(1) \sum_{1 \leq p_{1}, \ldots, p_{4} \leq d} \mathbb{E}_{\theta_{1, n}^{*}}\left[\prod_{j=1}^{4}\left(X_{i, p_{j}}-X_{i-1, p_{j}}-h_{n} b_{i-1, p_{j}}\left(\beta_{0}\right)\right) \mid \mathcal{G}_{i-1}^{n}\right] \\
& \leq R_{i-1}\left(h_{n}^{2}\right) .
\end{aligned}
$$

Therefore it holds

$$
0 \leq \sum_{i=1}^{n} \mathbb{E}_{\theta_{1, n}^{*}}\left[\frac{1}{\left(n h_{n}\right)^{2}}\left(\eta_{i, 1}^{m_{1}}\right)^{4} \mid \mathcal{G}_{i-1}^{n}\right] \leq \frac{1}{n} \sum_{i=1}^{n} \frac{1}{n} R_{i-1}(1) \stackrel{P}{\rightarrow} 0
$$

and

$$
\sum_{i=1}^{n} \mathbb{E}_{\theta_{1, n}^{*}}\left[\frac{1}{\left(n h_{n}\right)^{2}}\left(\eta_{i, 1}^{m_{1}}\right)^{4} \mid \mathcal{G}_{i-1}^{n}\right] \stackrel{P}{\rightarrow} 0
$$

Using $39-42$, we obtain 38 .

Next, one shows (ii). From Taylor's theorem with respect to $\beta$, we have

$$
-\frac{1}{\sqrt{n h_{n}}} \partial_{\beta} U_{n}^{(2)}\left(\beta_{0} \mid \hat{\alpha}_{n}\right)=\int_{0}^{1} \frac{1}{n h_{n}} \partial_{\beta}^{2} U_{n}^{(2)}\left(\beta_{0}+u\left(\hat{\beta}_{n}-\beta_{0}\right) \mid \hat{\alpha}_{n}\right) d u \sqrt{n h_{n}}\left(\hat{\beta}_{n}-\beta_{0}\right)
$$

and Taylor's theorem with respect to $\alpha$ yields that

$$
\begin{aligned}
\frac{1}{\sqrt{n h_{n}}} \partial_{\beta} U_{n}^{(2)}\left(\beta_{0} \mid \hat{\alpha}_{n}\right)= & \frac{1}{\sqrt{n h_{n}}} \partial_{\beta} U_{n}^{(2)}\left(\beta_{0} \mid \alpha_{0}\right) \\
& +\int_{0}^{1} \frac{1}{n \sqrt{h_{n}}} \partial_{\alpha \beta}^{2} U_{n}^{(2)}\left(\beta_{0} \mid \alpha_{0}+u\left(\hat{\alpha}_{n}-\alpha_{0}\right)\right) d u \sqrt{n}\left(\hat{\alpha}_{n}-\alpha_{0}\right) .
\end{aligned}
$$

One has that under $H_{0, n}^{(2)}$,

$$
\begin{aligned}
& \int_{0}^{1} \frac{1}{n h_{n}} \partial_{\beta}^{2} U_{n}^{(2)}\left(\beta_{0}+u\left(\hat{\beta}_{n}-\beta_{0}\right) \mid \hat{\alpha}_{n}\right) d u \stackrel{P}{\rightarrow}-I_{b}\left(\theta_{0} ; \theta_{0}\right), \\
& \int_{0}^{1} \frac{1}{n \sqrt{h_{n}}} \partial_{\alpha \beta}^{2} U_{n}^{(2)}\left(\beta_{0} \mid \alpha_{0}+u\left(\hat{\alpha}_{n}-\alpha_{0}\right)\right) d u \stackrel{P}{\rightarrow} 0
\end{aligned}
$$

which also hold under $H_{1, n}^{(2)}$ by C1. Therefore, it follows from (44) and 45 that

$$
-\int_{0}^{1} \frac{1}{n h_{n}} \partial_{\beta}^{2} U_{n}^{(2)}\left(\beta_{0}+u\left(\hat{\beta}_{n}-\beta_{0}\right) \mid \hat{\alpha}_{n}\right) d u \sqrt{n h_{n}}\left(\hat{\beta}_{n}-\beta_{0}\right)=\frac{1}{\sqrt{n h_{n}}} \partial_{\beta} U_{n}^{(2)}\left(\beta_{0} \mid \alpha_{0}\right)+o_{P}(1) .
$$


Since $I_{b}\left(\theta_{0} ; \theta_{0}\right)$ is non-singular, by Slutsky's theorem, one has that

$$
\sqrt{n h_{n}}\left(\hat{\beta}_{n}-\beta_{0}\right) \stackrel{d}{\rightarrow} I_{b}^{-1}\left(\theta_{0} ; \theta_{0}\right) L_{2}=Y_{2}^{\prime} \sim N\left(u_{\beta}, I_{b}^{-1}\left(\theta_{0} ; \theta_{0}\right)\right) \quad\left(\text { under } H_{1, n}^{(2)}\right) .
$$

This completes the proof of (ii).

Proof of Theorem 3. (i) By Taylor's theorem,

$$
U_{n}^{(1)}\left(\alpha_{0}\right)-U_{n}^{(1)}\left(\hat{\alpha}_{n}\right)=\left(\sqrt{n}\left(\hat{\alpha}_{n}-\alpha_{0}\right)\right)^{\top} J_{a, n}\left(\alpha_{0}, \hat{\alpha}_{n}\right) \sqrt{n}\left(\hat{\alpha}_{n}-\alpha_{0}\right) .
$$

It follows from Lemma 3 that

$$
\sqrt{n}\left(\hat{\alpha}_{n}-\alpha_{0}\right) \stackrel{d}{\rightarrow} Y_{1}^{\prime} \sim N\left(u_{\alpha}, I_{a}^{-1}\left(\alpha_{0} ; \alpha_{0}\right)\right) \quad\left(\text { under } H_{1, n}^{(1)}\right) .
$$

Moreover, $\mathbf{C 1}$ and

$$
J_{a, n}\left(\alpha_{0}, \hat{\alpha}_{n}\right) \stackrel{P}{\rightarrow}-\frac{1}{2} I_{a}\left(\alpha_{0} ; \alpha_{0}\right) \quad\left(\text { under } H_{0, n}^{(1)}\right)
$$

implies

$$
J_{a, n}\left(\alpha_{0}, \hat{\alpha}_{n}\right) \stackrel{P}{\rightarrow}-\frac{1}{2} I_{a}\left(\alpha_{0} ; \alpha_{0}\right) \quad\left(\text { under } H_{1, n}^{(1)}\right) .
$$

By noting that $Y_{1}^{\prime}=I_{a}^{-\frac{1}{2}}\left(\alpha_{0} ; \alpha_{0}\right) Z_{1}^{\prime}, Z_{1}^{\prime} \sim N\left(I_{a}^{\frac{1}{2}}\left(\alpha_{0} ; \alpha_{0}\right) u_{\alpha}, E_{p_{1}}\right)$, it follows from the continuous mapping theorem that

$$
\begin{aligned}
\Lambda_{n}^{(1)} & =-2\left(U_{n}^{(1)}\left(\alpha_{0}\right)-U_{n}^{(1)}\left(\hat{\alpha}_{n}\right)\right) \\
& \stackrel{d}{\rightarrow} Y_{1}^{\prime \top} I_{a}\left(\alpha_{0} ; \alpha_{0}\right) Y_{1}^{\prime} \\
& =Z_{1}^{\prime \top} Z_{1}^{\prime} \\
& \sim \chi_{p_{1}}^{2}\left(u_{\alpha}^{\top} I_{a}\left(\alpha_{0} ; \alpha_{0}\right) u_{\alpha}\right) \quad\left(\text { under } H_{1, n}^{(1)}\right) .
\end{aligned}
$$

Moreover, by Taylor's theorem with respect to $\beta$, we have

$$
U_{n}^{(2)}\left(\beta_{0} \mid \hat{\alpha}_{n}\right)-U_{n}^{(2)}\left(\hat{\beta}_{n} \mid \hat{\alpha}_{n}\right)=\left(\sqrt{n h_{n}}\left(\hat{\beta}_{n}-\beta_{0}\right)\right)^{\top} J_{b, n}\left(\beta_{0}, \hat{\beta}_{n}\right) \sqrt{n h_{n}}\left(\hat{\beta}_{n}-\beta_{0}\right) .
$$

It follows from Lemma 3 that

$$
\sqrt{n h_{n}}\left(\hat{\beta}_{n}-\beta_{0}\right) \stackrel{d}{\rightarrow} Y_{2}^{\prime} \sim N\left(u_{\beta}, I_{b}^{-1}\left(\theta_{0} ; \theta_{0}\right)\right) \quad\left(\text { under } H_{1, n}^{(2)}\right) .
$$

Next, we can check

$$
J_{b, n}\left(\beta_{0}, \hat{\beta}_{n}\right) \stackrel{P}{\rightarrow}-\frac{1}{2} I_{b}\left(\theta_{0} ; \theta_{0}\right) \quad\left(\text { under } H_{1, n}^{(2)}\right) .
$$

Note that $Y_{2}^{\prime}=I_{b}^{-\frac{1}{2}}\left(\theta_{0} ; \theta_{0}\right) Z_{2}^{\prime}, Z_{2}^{\prime} \sim N\left(I_{b}^{\frac{1}{2}}\left(\theta_{0} ; \theta_{0}\right) u_{\beta}, E_{p_{2}}\right)$. By the continuous mapping theorem, one has that

$$
\begin{aligned}
\Lambda_{n}^{(2)} & =-2\left(U_{n}^{(2)}\left(\beta_{0} \mid \hat{\alpha}_{n}\right)-U_{n}^{(2)}\left(\hat{\beta}_{n} \mid \hat{\alpha}_{n}\right)\right) \\
& \stackrel{d}{\rightarrow} Y_{2}^{\prime \top} I_{b}\left(\theta_{0} ; \theta_{0}\right) Y_{2}^{\prime} \\
& =Z_{2}^{\prime \top} Z_{2}^{\prime} \\
& \sim \chi_{p_{2}}^{2}\left(u_{\beta}^{\top} I_{b}\left(\theta_{0} ; \theta_{0}\right) u_{\beta}\right) \quad\left(\text { under } H_{1, n}^{(2)}\right) .
\end{aligned}
$$

This completes the proof of the Likelihood type.

(ii) From C1, we have

$$
I_{a, n}\left(\hat{\alpha}_{n}\right) \stackrel{P}{\rightarrow} I_{a}\left(\alpha_{0} ; \alpha_{0}\right) \quad\left(\text { under } H_{1, n}^{(1)}\right) .
$$

Therefore, it follows from Lemma 3 and the continuous mapping theorem that

$$
\begin{aligned}
W_{n}^{(1)} & =\left(\sqrt{n}\left(\hat{\alpha}_{n}-\alpha_{0}\right)\right)^{\top} I_{a, n}\left(\hat{\alpha}_{n}\right) \sqrt{n}\left(\hat{\alpha}_{n}-\alpha_{0}\right) \\
& \stackrel{d}{\rightarrow} Y_{1}^{\prime \top} I_{a}\left(\alpha_{0} ; \alpha_{0}\right) Y_{1}^{\prime} \\
& \sim \chi_{p_{1}}^{2}\left(u_{\alpha}^{\top} I_{a}\left(\alpha_{0} ; \alpha_{0}\right) u_{\alpha}\right) \quad\left(\text { under } H_{1, n}^{(1)}\right) .
\end{aligned}
$$


Furthermore, we obtain

$$
I_{b, n}\left(\hat{\beta}_{n} \mid \hat{\alpha}_{n}\right) \stackrel{P}{\rightarrow} I_{b}\left(\theta_{0} ; \theta_{0}\right) \quad\left(\text { under } H_{0, n}^{(2)}\right),
$$

and

$$
\begin{aligned}
W_{n}^{(2)} & =\left(\sqrt{n h_{n}}\left(\hat{\beta}_{n}-\beta_{0}\right)\right)^{\top} I_{b, n}\left(\hat{\beta}_{n} \mid \hat{\alpha}_{n}\right) \sqrt{n h_{n}}\left(\hat{\beta}_{n}-\beta_{0}\right) \\
& \stackrel{d}{\rightarrow} Y_{2}^{\prime \top} I_{b}\left(\theta_{0} ; \theta_{0}\right) Y_{2}^{\prime} \\
& \sim \chi_{p_{2}}^{2}\left(u_{\beta}^{\top} I_{b}\left(\theta_{0} ; \theta_{0}\right) u_{\beta}\right) \quad\left(\text { under } H_{1, n}^{(2)}\right) .
\end{aligned}
$$

This completes the proof of the Wald type.

(iii) From the proof of Lemma 3 , it is shown that

$$
\frac{1}{\sqrt{n}} \partial_{\alpha} U_{n}^{(1)}\left(\alpha_{0}\right) \stackrel{d}{\rightarrow} L_{1}=I_{a}\left(\alpha_{0} ; \alpha_{0}\right) Y_{1}^{\prime} \sim N\left(I_{a}\left(\alpha_{0} ; \alpha_{0}\right) u_{\alpha}, I_{a}\left(\alpha_{0} ; \alpha_{0}\right)\right) \quad\left(\text { under } H_{1, n}^{(1)}\right) .
$$

On the other hand, we can check

$$
\bar{I}_{a, n}\left(\hat{\alpha}_{n}\right) \stackrel{P}{\rightarrow} I_{a}^{-1}\left(\alpha_{0} ; \alpha_{0}\right) \quad\left(\text { under } H_{1, n}^{(1)}\right) .
$$

Therefore, it follows from the continuous mapping theorem that

$$
\begin{aligned}
R_{n}^{(1)} & =\left(\frac{1}{\sqrt{n}} \partial_{\alpha} U_{n}^{(1)}\left(\alpha_{0}\right)\right)^{\top} \bar{I}_{a, n}\left(\hat{\alpha}_{n}\right) \frac{1}{\sqrt{n}} \partial_{\alpha} U_{n}^{(1)}\left(\alpha_{0}\right) \\
& \stackrel{d}{\rightarrow} L_{1}^{\top} I_{a}^{-1}\left(\alpha_{0} ; \alpha_{0}\right) L_{1} \\
& =Y_{1}^{\prime \top} I_{a}\left(\alpha_{0} ; \alpha_{0}\right) Y_{1}^{\prime} \\
& \sim \chi_{p_{1}}^{2}\left(u_{\alpha}^{\top} I_{a}\left(\alpha_{0} ; \alpha_{0}\right) u_{\alpha}\right) \quad\left(\text { under } H_{1, n}^{(1)}\right) .
\end{aligned}
$$

Moreover, it follows from the proof of Lemma 3 that

$$
\frac{1}{\sqrt{n h_{n}}} \partial_{\beta} U_{n}^{(2)}\left(\beta_{0} \mid \alpha_{0}\right) \stackrel{d}{\rightarrow} L_{2}=I_{b}\left(\theta_{0} ; \theta_{0}\right) Y_{2}^{\prime} \sim N\left(I_{b}\left(\theta_{0} ; \theta_{0}\right) u_{\beta}, I_{b}\left(\theta_{0} ; \theta_{0}\right)\right) \quad\left(\text { under } H_{1, n}^{(2)}\right) .
$$

By $\mathbf{C 1}$ and Taylor's theorem with respect to $\alpha$, we have

$$
\begin{aligned}
\frac{1}{\sqrt{n h_{n}}} \partial_{\beta} U_{n}^{(2)}\left(\beta_{0} \mid \hat{\alpha}_{n}\right)= & \frac{1}{\sqrt{n h_{n}}} \partial_{\beta} U_{n}^{(2)}\left(\beta_{0} \mid \alpha_{0}\right) \\
& +\int_{0}^{1} \frac{1}{n \sqrt{h_{n}}} \partial_{\alpha} \partial_{\beta} U_{n}^{(2)}\left(\beta_{0} \mid \alpha_{0}+u\left(\hat{\alpha}_{n}-\alpha_{0}\right)\right) d u \sqrt{n}\left(\hat{\alpha}_{n}-\alpha_{0}\right) \\
= & \frac{1}{\sqrt{n h_{n}}} \partial_{\beta} U_{n}^{(2)}\left(\beta_{0} \mid \alpha_{0}\right)+o_{P}(1) \quad\left(\text { under } H_{1, n}^{(2)}\right) .
\end{aligned}
$$

Next, we can show that

$$
\bar{I}_{b, n}\left(\hat{\beta}_{n} \mid \hat{\alpha}_{n}\right) \stackrel{P}{\rightarrow} I_{b}^{-1}\left(\theta_{0} ; \theta_{0}\right) \quad\left(\text { under } H_{1, n}^{(2)}\right)
$$

Therefore, by the continuous mapping theorem, it holds

$$
\begin{aligned}
R_{n}^{(2)} & =\left(\frac{1}{\sqrt{n h_{n}}} \partial_{\beta} U_{n}^{(2)}\left(\beta_{0} \mid \hat{\alpha}_{n}\right)\right)^{\top} \bar{I}_{b, n}\left(\hat{\beta}_{n} \mid \hat{\alpha}_{n}\right) \frac{1}{\sqrt{n h_{n}}} \partial_{\beta} U_{n}^{(2)}\left(\beta_{0} \mid \hat{\alpha}_{n}\right) \\
& \stackrel{d}{\rightarrow} L_{2}^{\top} I_{b}^{-1}\left(\theta_{0} ; \theta_{0}\right) L_{2} \\
& =Y_{2}^{\prime \top} I_{b}\left(\theta_{0} ; \theta_{0}\right) Y_{2}^{\prime} \\
& \sim \chi_{p_{2}}^{2}\left(u_{\beta}^{\top} I_{b}\left(\theta_{0} ; \theta_{0}\right) u_{\beta}\right) \quad\left(\text { under } H_{1, n}^{(2)}\right),
\end{aligned}
$$

which completes the proof of the Rao type. 


\section{REFERENCES}

[1] A. De Gregorio and S. M. Iacus. On a family of test statistics for discretely observed diffusion processes. Journal of Multivariate Analysis, 122:292-316, 2013.

[2] A. De Gregorio and S. M. Iacus. Empirical $L^{2}$-distance test statistics for ergodic diffusions. Statistical Inference for Stochastic Processes, 22(2):233-261, 2019.

[3] T. S. Ferguson. A course in large sample theory. Chapman \& Hall, London, 1996.

[4] D. Florens-Zmirou. Approximate discrete-time schemes for statistics of diffusion processes. Statistics: A Journal of Theoretical and Applied Statistics, 20(4):547-557, 1989.

[5] V. Genon-Catalot and J. Jacod. On the estimation of the diffusion coefficient for multi-dimensional diffusion processes. In Annales de l'IHP Probabilités et statistiques, volume 29, pages 119-151, 1993.

[6] E. Gobet. LAN property for ergodic diffusions with discrete observations. In Annales de l'IHP Probabilités et statistiques, volume 38, pages 711-737, 2002.

[7] P. Hall and C. C. Heyde. Martingale limit theory and its application. Academic press, New York, 1980.

[8] M. Kessler. Estimation des paramètres d'une diffusion par des contrastes corrigés. Comptes Rendus de l'Académie des Sciences - Series I - Mathematics, 320:359-362, 1995.

[9] M. Kessler. Estimation of an ergodic diffusion from discrete observations. Scandinavian Journal of Statistics, 24(2):211-229, 1997.

[10] H. Kitagawa and M. Uchida. Adaptive test statistics for ergodic diffusion processes sampled at discrete times. Journal of Statistical Planning and Inference, 150:84-110, 2014.

[11] E. Lehmann. Elements of Large-Sample Theory. Springer Texts in Statistics. Springer-Verlag, New York, 1999.

[12] S. H. Nakakita and M. Uchida. Adaptive test for ergodic diffusions plus noise. Journal of Statistical Planning and Inference, 203:131-150, 2019.

[13] M. Uchida and N. Yoshida. Adaptive estimation of an ergodic diffusion process based on sampled data. Stochastic Processes and their Applications, 122(8):2885-2924, 2012.

[14] A. W. Van der Vaart. Asymptotic statistics. Cambridge university press, Cambridge, 1998.

[15] N. Yoshida. Estimation for diffusion processes from discrete observation. Journal of Multivariate Analysis, 41(2):220-242, 1992.

[16] N. Yoshida. Polynomial type large deviation inequalities and quasi-likelihood analysis for stochastic differential equations. Annals of the Institute of Statistical Mathematics, 63:431-479, 2011. 